\title{
The tempered spectrum of a real spherical space
}

\author{
by \\ FRIEDRICH KNOP \\ Emmy-Noether Zentrum \\ Erlangen, Germany \\ BERNHARD KRÖTZ \\ Universität Paderborn \\ Paderborn, Germany \\ HENRIK SCHLICHTKRULL \\ University of Copenhagen \\ Copenhagen, Denmark
}

\section{Introduction}

For a real reductive group $G$ one important step towards the Plancherel theorem is the determination of the support of the Plancherel measure, i.e. the portion of the unitary dual of $G$ which is contained weakly in $L^{2}(G)$. It turns out that these representations are the so-called tempered representations, i.e. representations whose matrix coefficients satisfy a certain moderate growth condition. Further, one has the central theorem of Langlands.

TEMPERED EMBEDDING THEOREM. ([28]) Every irreducible tempered representation $\pi$ is induced from discrete series, i.e. there is a parabolic subgroup $P<G$ with Langlands decomposition $P=M A N$, a discrete series representation $\sigma$ of $M$ and a unitary character $\chi$ of $A$ such that $\pi$ is a subrepresentation of $\operatorname{Ind}_{P}^{G}(\sigma \otimes \chi \times \mathbf{1})$.

Thus (up to equivalence) the description of the tempered spectrum is reduced to the classification of discrete series representations. A generalization with an analogous formulation was obtained by Delorme [12] for symmetric spaces $G / H$.

In this article we consider the more general case of real spherical spaces, that is, homogeneous spaces $Z=G / H$ on which the minimal parabolic subgroups of $G$ admit open orbits. This case is more complicated and several standard techniques from the previous cases cannot be applied. The result which in its formulation comes closest to the theorem above is obtained for a particular class of real spherical spaces, said to be of wave-front type. The spaces of this type feature a simplified large scale geometry [21]

The second author was supported by ERC Advanced Investigators Grant HARG 268105 
and satisfy the wave-front lemma of Eskin-McMullen [13], [21]. In particular, they are well suited for lattice counting problems [25]. The notion was originally introduced by Sakellaridis and Venkatesh [34].

Another large class of real spherical spaces $Z=G / H$ is obtained by taking real forms of complex spherical spaces $Z_{\mathbb{C}}=G_{\mathbb{C}} / H_{\mathbb{C}}$. We call those $Z$ absolutely spherical. A generalization of Langlands' theorem will be obtained also for this class of spaces. It should be noted that all symmetric spaces are both absolutely spherical and of wavefront type, but there exist real spherical spaces which do not satisfy one or both of these properties.

Complex spherical spaces $Z_{\mathbb{C}}$ with $H_{\mathbb{C}}$ reductive were classified by Krämer [23] (for $G_{\mathbb{C}}$ simple) and Brion-Mikityuk [6], [32] (for $G_{\mathbb{C}}$ semi-simple). Recently we obtained a classification for all real spherical spaces $Z$ for $H$ reductive (see [18], [19]). In particular, if $G$ is simple, it turns out that there are only few cases which are neither absolutely spherical nor wave-front; concretely these are $\operatorname{SL}(n, \mathbb{H}) / \operatorname{SL}(n-1, \mathbb{H})$ for $n \geqslant 3$, $\mathrm{SO}(4,7) /(\mathrm{SO}(3) \times \mathrm{Spin}(3,4))$, and $\mathrm{SO}(3,6) /\left(\mathrm{G}_{2}^{1} \times \mathrm{SO}(2)\right)$.

Let now $Z=G / H$ be a unimodular real spherical space. On the geometric level we attach to $Z$ a finite set of equal-dimensional boundary degenerations $Z_{I}=G / H_{I}$. These boundary degenerations are real spherical homogeneous spaces parametrized by subsets $I$ of the set of spherical roots $S$ attached to $Z$.

In the group case $Z=(G \times G) / \operatorname{diag}(G) \simeq G$ the boundary degenerations are of the form $Z_{P}=(G \times G) /\left(\left(N \times N^{\text {opp }}\right) \operatorname{diag}\left(M_{0} A\right)\right)$ attached to an arbitrary parabolic subgroup $P=M A N<G$. We remark that, up to conjugation, parabolic subgroups are parametrized by finite subsets of the set of simple restricted roots.

To a Harish-Chandra module $V$ and a continuous $H$-invariant functional $\eta$ (on some completion of $V$ ) we attach a leading exponent $\Lambda_{V, \eta}$, and we provide a necessary and sufficient criterion, in terms of this leading exponent, for the pair $(V, \eta)$ to belong to a twisted discrete series of $Z$, induced from a unitary character of $H$.

In [21] we defined tempered pairs $(V, \eta)$. Under the assumption that $Z$ is absolutely spherical or wave-front, the main result of this paper is then that for every tempered pair $(V, \eta)$ there exists a boundary degeneration $Z_{I}$ of $Z$ and an equivariant embedding of $V$ into the twisted discrete series of $Z_{I}$.

In the special case of real spherical space of wave-front type, our result gives rise to a tempered embedding theorem in the more familiar formulation of parabolic induction; see Corollary 9.13. In the case of $p$-adic wave-front space, such an embedding theorem was proved by Sakellaridis-Venkatesh [34]. 


\subsection{The composition of the paper}

There are two parts. A geometric part in $\S \S 2-5$, and an analytic part in $\S \S 6-9$.

The geometric part begins with the construction of the boundary degenerations $Z_{I}$ of $Z$. Here we choose an algebraic approach which gives the deformations of $\mathfrak{h}:=\operatorname{Lie}(H)$ into $\mathfrak{h}_{I}:=\operatorname{Lie}\left(H_{I}\right)$ via a simple limiting procedure. Then, in $\S 3$, we relate the polar decomposition (see [20]) of $Z$ and $Z_{I}$ via the standard compactifications (see [17]). This is rather delicate, as representatives of open $P_{\min }$-orbits on $Z$ (and $Z_{I}$ ) naturally enter the polar decompositions and these representatives need to be carefully chosen. $\S 4$ is concerned with real spherical spaces which we call induced: For any parabolic $P<G$ with Levi decomposition $P=G_{P} U_{P}$, we obtain an induced real spherical space $Z_{P}=G_{P} / H_{P}$, where $H_{P}<G_{P}$ is the projection of $P \cap H$ to $G_{P}$ along $U_{P}$. We conclude the geometric half with a treatment of wave-front spaces and elaborate on their special geometry.

The analytic part starts with power series expansions for generalized matrix coefficients on $Z$. We show that the generalized matrix coefficients are solutions of a certain holonomic regular singular system of differential equations extending the results of $[27, \S 5]$.

For every continuous $\mathfrak{h}$-invariant functional $\eta$ on $V$, we then construct $\mathfrak{h}_{I}$-invariant functionals $\eta_{I}$ on $V$ by extracting certain parts of the power series expansion. Passing from $\eta$ to $\eta_{I}$ can be considered as an algebraic version of the Radon transform. The technically most difficult part of this paper is then to establish the continuity of $\eta_{I}$. For symmetric spaces this would not be an issue in view of the automatic continuity theorem by van den Ban, Brylinski and Delorme [3], [8]. For real spherical spaces such a result is currently not available. Under the assumption that $Z$ is either absolutely spherical or wave-front, we settle this issue in Theorems 7.2 and 7.6, via quite delicate optimal upper and lower bounds for the generalized matrix coefficients. Already in the group case, these bounds provide a significant improvement of the standard results (see Remark 7.4). The proof is based on the comparison theorems from [9], [6], and [1].

Acknowledgement. It is our pleasure to thank Patrick Delorme for many invaluable comments to earlier versions of this paper.

\section{Real spherical spaces}

A standing convention of this paper is that (real) Lie groups will be denoted by upper case Latin letters, e.g. $A, B$, etc., and their Lie algebras by lower case German letters, e.g. $\mathfrak{a}, \mathfrak{b}$, etc. The identity component of a Lie group $G$ will be denoted by $G_{e}$.

Let $G$ be an algebraic real reductive group by which we understand an open subgroup 
of the real points of a connected complex reductive algebraic group $G_{\mathbb{C}}$. Let $H<G$ be a closed connected subgroup such that there is a complex algebraic subgroup $H_{\mathbb{C}}<G_{\mathbb{C}}$ such that $H=\left(G \cap H_{\mathbb{C}}\right)_{e}$. Under these assumptions we refer to $Z=G / H$ as a real algebraic homogeneous space. We set $Z_{\mathbb{C}}=G_{\mathbb{C}} / H_{\mathbb{C}}$ and note that there is a natural $G$-equivariant morphism

$$
\begin{gathered}
Z \longrightarrow Z_{\mathbb{C}}, \\
g H \longmapsto g H_{\mathbb{C}} .
\end{gathered}
$$

Let us denote by $z_{0}=H$ the standard base point of $Z$.

We assume that $Z$ is real spherical, i.e. we assume that a minimal parabolic subgroup $P_{\min }<G$ admits an open orbit on $Z$. It is no loss of generality to request that $P_{\min } H \subset G$ is open, or equivalently

$$
\mathfrak{g}=\mathfrak{p}_{\min }+\mathfrak{h} .
$$

If $L$ is a real algebraic group, then we denote by $L_{\mathrm{n}} \triangleleft L$ the connected normal subgroup generated by all unipotent elements.

According to the local structure theorem of [22], there exists a unique parabolic subgroup $Q \supset P_{\min }$ (called $Z$-adapted), with a Levi decomposition $Q=L U$ such that

- $P_{\min } \cdot z_{0}=Q \cdot z_{0}$,

- $L_{\mathrm{n}}<Q \cap H<L$.

We let $K_{L} A_{L} N_{L}=L$ be an Iwasawa decomposition of $L$ with $N_{L}<P_{\min }$, set $A:=A_{L}$ and obtain a Levi decomposition $P_{\min }=M A N=M A \ltimes N$, where $M=Z_{K_{L}}(A)$ and $N=$ $N_{L} U$. We set $A_{H}:=A \cap H$, and further

$$
A_{Z}:=A / A_{H}
$$

The dimension of $A_{Z}$ is an invariant of the real spherical space, called its real rank; in symbols $\operatorname{rank}_{\mathbb{R}}(Z)$. Observe that it follows from the fact that $\mathfrak{l}_{\mathrm{n}} \subset \mathfrak{h}$ that

$$
\mathfrak{a}=\mathfrak{a} \cap \mathfrak{z}(\mathfrak{l})+\mathfrak{a} \cap \mathfrak{h},
$$

where $\mathfrak{z}(\mathfrak{l})$ denotes the center of $\mathfrak{l}$. In particular, it follows that $\mathfrak{l} \cap \mathfrak{h}$ is $\operatorname{Ad}(A)$-invariant.

We extend $K_{L}$ to a maximal compact subgroup $K$ of $G$ and denote by $\theta$ the corresponding Cartan involution. Further we put $\overline{\mathfrak{n}}:=\theta(\mathfrak{n})$ and $\overline{\mathfrak{u}}:=\theta(\mathfrak{u})$. Later in this article it will be convenient to replace $K$ by $K^{a}:=a K a^{-1}$ for a suitable element $a \in A$. Then $\theta$ becomes replaced by $\theta^{a}:=\operatorname{Ad}(a) \circ \theta \circ \operatorname{Ad}(a)^{-1}$, and for this reason it is important to monitor the dependence of our definitions on $\theta$. For example, we note that $M, \overline{\mathfrak{n}}$ and $\overline{\mathfrak{u}}$ are unaltered by such a change. 
We fix an invariant non-degenerate bilinear form $\varkappa$ on $\mathfrak{g}$. Associated with that, we have the inner product $\langle X, Y\rangle:=\varkappa(X, \theta Y)$ on $\mathfrak{g}$, called the Cartan-Killing form. Note that it depends on the Cartan involution.

We write $\Sigma=\Sigma(\mathfrak{g}, \mathfrak{a}) \subset \mathfrak{a}^{*} \backslash\{0\}$ for the restricted root system attached to the pair $(\mathfrak{g}, \mathfrak{a})$. For $\alpha \in \Sigma$ we denote by $\mathfrak{g}^{\alpha}$ the corresponding root space and write

$$
\mathfrak{g}=\mathfrak{a} \oplus \mathfrak{m} \oplus \bigoplus_{\alpha \in \Sigma} \mathfrak{g}^{\alpha}
$$

for the decomposition of $\mathfrak{g}$ into root spaces. Here, as usual, $\mathfrak{m}$ is the Lie algebra of $M$.

\subsection{Examples of real spherical spaces}

If $\mathfrak{h}<\mathfrak{g}$ is a subalgebra such that there exists a minimal parabolic subalgebra $\mathfrak{p}_{\text {min }}$ such that $\mathfrak{g}=\mathfrak{h}+\mathfrak{p}_{\min }$, then we call $(\mathfrak{g}, \mathfrak{h})$ a real spherical pair and $\mathfrak{h}$ a real spherical subalgebra of $\mathfrak{g}$. A subalgebra $\mathfrak{h}<\mathfrak{g}$ is called symmetric if there exists an involutive automorphism $\tau: \mathfrak{g} \rightarrow \mathfrak{g}$ with fixed-point set $\mathfrak{h}$. We recall that every symmetric subalgebra is reductive and that every symmetric subalgebra is real spherical. Symmetric subalgebras have been classified by Cartan and Berger.

A pair $(\mathfrak{g}, \mathfrak{h})$ of a complex Lie algebra and a complex subalgebra is called complex spherical or simply spherical if it is real spherical when regarded as a pair of real Lie algebras. Note that in this case the minimal parabolic subalgebras of $\mathfrak{g}$ are precisely the Borel subalgebras.

LEMma 2.1. Let $\mathfrak{h}<\mathfrak{g}$ be a subalgebra such that $\left(\mathfrak{g}_{\mathbb{C}}, \mathfrak{h}_{\mathbb{C}}\right)$ is a complex spherical pair. Then $(\mathfrak{g}, \mathfrak{h})$ is a real spherical pair.

Proof. Let $\mathfrak{b}_{\mathbb{C}}$ be a Borel subalgebra of $\mathfrak{g}_{\mathbb{C}}$ which is contained in $\mathfrak{p}_{\min , \mathbb{C}}$. We claim that there exists a $g \in G$ such that $\mathfrak{h}_{\mathbb{C}}+\operatorname{Ad}(g) \mathfrak{b}_{\mathbb{C}}=\mathfrak{g}_{\mathbb{C}}$. This follows immediately from the fact that $\left(\mathfrak{g}_{\mathbb{C}}, \mathfrak{h}_{\mathbb{C}}\right)$ is a spherical pair, since the set of elements $g \in G_{\mathbb{C}}$ for which $\mathfrak{h}_{\mathbb{C}}+\operatorname{Ad}(g) \mathfrak{b}_{\mathbb{C}}=\mathfrak{g}_{\mathbb{C}}$ is then non-empty, Zariski open and defined over $\mathbb{R}$.

Let $g \in G$ be such an element. It follows that $\mathfrak{h}_{\mathbb{C}}+\operatorname{Ad}(g) \mathfrak{p}_{\text {min, }, \mathbb{C}}=\mathfrak{g}_{\mathbb{C}}$, and taking real points this implies that $\mathfrak{h}+\operatorname{Ad}(g) \mathfrak{p}_{\min }=\mathfrak{g}$.

We note that the converse of the lemma is not true if $\mathfrak{g}$ is not quasi-split. For example $(\mathfrak{g}, \mathfrak{n})$ is a real spherical pair, but the complexification $\left(\mathfrak{g}_{\mathbb{C}}, \mathfrak{n}_{\mathbb{C}}\right)$ is not spherical unless $\mathfrak{g}$ is quasi-split. The real spherical pairs $(\mathfrak{g}, \mathfrak{h})$ obtained from complex spherical pairs $\left(\mathfrak{g}_{\mathbb{C}}, \mathfrak{h}_{\mathbb{C}}\right)$ are called absolutely spherical or real forms. 


\begin{tabular}{|c|c|}
\hline $\mathfrak{g}_{\mathbb{C}}$ & $\mathfrak{h}_{\mathbb{C}}$ \\
\hline \hline $\mathfrak{s l}(n, \mathbb{C})$ & $\mathfrak{s l}(p, \mathbb{C}) \oplus \mathfrak{s l}(n-p, \mathbb{C}), 2 p \neq n$ \\
$\mathfrak{s l}(2 n+1, \mathbb{C})$ & $\mathfrak{s p}(n, \mathbb{C}) \oplus \mathbb{C}$ \\
$\mathfrak{s l}(2 n+1, \mathbb{C})$ & $\mathfrak{s p}(n, \mathbb{C})$ \\
\hline $\mathfrak{s o}(2 n+1, \mathbb{C})$ & $\mathfrak{g l}(n, \mathbb{C})$ \\
$\mathfrak{s o}(9, \mathbb{C})$ & $\mathfrak{s p i n}(7, \mathbb{C})$ \\
$\mathfrak{s o}(7, \mathbb{C})$ & $G_{2}$ \\
\hline $\mathfrak{s p}(2 n, \mathbb{C})$ & $\mathfrak{s p}(n-1, \mathbb{C}) \oplus \mathbb{C}$ \\
\hline $\mathfrak{s o}(2 n, \mathbb{C})$ & $\mathfrak{s l}(n, \mathbb{C}), n$ odd \\
$\mathfrak{s o}(10, \mathbb{C})$ & $\mathfrak{s o}(2, \mathbb{C}) \oplus \mathfrak{s p i n}(7, \mathbb{C})$ \\
$\mathfrak{s o}(8, \mathbb{C})$ & $G_{2}$ \\
\hline$G_{2}$ & $\mathfrak{s l}(3, \mathbb{C})$ \\
$E_{6}$ & $\mathfrak{s o}(10, \mathbb{C})$ \\
\hline
\end{tabular}

Table 1. The non-symmetric cases of Krämer's list.

\subsubsection{Examples of absolutely spherical pairs with $\mathfrak{h}$ reductive}

Complex spherical pairs $\left(\mathfrak{g}_{\mathbb{C}}, \mathfrak{h}_{\mathbb{C}}\right)$ with $\mathfrak{h}_{\mathbb{C}}$ reductive have been classified. For $\mathfrak{g}_{\mathbb{C}}$ simple this goes back to Krämer [23], and it was extended to the semi-simple case by Brion [7] and Mikityuk [32]. For convenience, we recall the non-symmetric cases of Krämer's list in Table 1.

The pairs in the table feature plenty of non-compact real forms, classified in [18] and [19]. For example, the pairs $(\mathfrak{s l}(2 n+1, \mathbb{C}), \mathfrak{s p}(n, \mathbb{C})),(\mathfrak{s o}(2 n+1, \mathbb{C}), \mathfrak{g l}(n, \mathbb{C}))$ and $\left(\mathfrak{s o}(7, \mathbb{C}), G_{2}\right)$ have the following non-compact real forms:

$$
\begin{aligned}
& (\mathfrak{s u}(2 p, 2 q+1), \mathfrak{s p}(p, q)) \quad \text { and } \quad(\mathfrak{s l}(2 n+1, \mathbb{R}), \mathfrak{s p}(n, \mathbb{R})), \\
& (\mathfrak{s o}(n, n+1), \mathfrak{g l}(n, \mathbb{R})), \\
& \left(\mathfrak{s o}(3,4), \mathrm{G}_{2}^{1}\right), \quad \text { where } \mathrm{G}_{2}^{1} \text { is the split real form of } G_{2} .
\end{aligned}
$$

From the list of irreducible complex spherical pairs $\left(\mathfrak{g}_{\mathbb{C}}, \mathfrak{h}_{\mathbb{C}}\right)$ with $\mathfrak{g}_{\mathbb{C}}$ non-simple (see [7], [32]), we highlight the Gross-Prasad cases:

$$
\begin{aligned}
& (\mathfrak{s l}(n+1, \mathbb{C}) \oplus \mathfrak{s l}(n, \mathbb{C}), \mathfrak{g l}(n, \mathbb{C})), \\
& (\mathfrak{s o}(n+1, \mathbb{C}) \oplus \mathfrak{s o}(n, \mathbb{C}), \mathfrak{s o}(n, \mathbb{C})),
\end{aligned}
$$

which are ubiquitous in automorphic forms [15]. 


\subsubsection{Real spherical pairs which are not absolutely spherical}

Prominent examples are constituted by the triple spaces

$$
(\mathfrak{g}, \mathfrak{h})=(\mathfrak{h} \times \mathfrak{h} \times \mathfrak{h}, \operatorname{diag} \mathfrak{h}) \quad \text { for } \mathfrak{h}=\mathfrak{s o}(1, n),
$$

which are real spherical for $n \geqslant 2$ but not absolutely spherical when $n \geqslant 4$ (see [5], [11]). This example will be discussed later in the context of Levi-induced spaces in §4.2.2.

Other interesting examples for $\mathfrak{g}$ simple are (see [18]) the following:

- $\left(\mathrm{E}_{7}^{2}, \mathrm{E}_{6}^{2}\right),\left(\mathrm{E}_{7}^{2}, \mathrm{E}_{6}^{3}\right),\left(\mathrm{E}_{6}^{4}, \mathfrak{s l}(3, \mathbb{H})\right)$;

- $(\mathfrak{s l}(n, \mathbb{H}), \mathfrak{s l}(n-1, \mathbb{H})),(\mathfrak{s o}(2 p, 2 q), \mathfrak{s u}(p, q))$ for $p \neq q$;

- $\left(\mathfrak{s o}(6,3), \mathfrak{s o}(2) \oplus \mathrm{G}_{2}^{1}\right)$ and $(\mathfrak{s o}(7,4), \mathfrak{s p i n}(4,3)+\mathfrak{s o}(3))$.

\subsubsection{Non-reductive examples}

We begin with a general fact (see [7, Proposition 1.1] for a slightly weaker statement in the complex case).

Proposition 2.2. Let $P<G$ be a parabolic subgroup and $H<P$ be an algebraic subgroup. Let $P=L_{P} \ltimes U_{P}$ be a Levi decomposition of $P$. Then the following statements are equivalent:

(1) $Z=G / H$ is real spherical;

(2) $P / H$ is an $L_{P}$-spherical variety, i.e. the action of a minimal parabolic subgroup of $L_{P}$ admits an open orbit on $P / H$.

Proof. Let $P^{\mathrm{opp}}<G$ be the parabolic subgroup of $G$ with $P^{\mathrm{opp}} \cap P=L_{P}$, and let $P_{\min }<P^{\text {opp }}$ be a minimal parabolic subgroup of $G$. By the Bruhat decomposition, we have that $P_{\min } P$ is the only open double $\left(P_{\min } \times P\right)$-coset in $G$. Moreover, $S:=P_{\min } \cap P$ is a minimal parabolic subgroup of $L_{P}$.

Assume (1) and let $g \in G$ be such that $P_{\min } g H$ is open in $G$. Then $P_{\min } g P$ is also open in $G$, and hence $g \in P_{\min } P$. We may thus assume $g \in P$. Then $P_{\min } g H \cap P=S g H$ is open in $P$, proving (2).

Assume (2) and let $p \in P$ be such that $S p H$ is open in $P$. Then $P_{\min } p H=P_{\min } S p H$ is open in $P_{\min } P$ and in $G$, proving (1).

Example 2.3. We consider the group $G=\mathrm{SU}(p, q)$ with $3 \leqslant p \leqslant q$. This group has real rank $p$ with restricted root system $B C_{p}$ or $C_{p}$ (if $p=q$ ). Let $P=P_{\min }=M A N$ be a minimal subgroup. Let $N_{0}<N$ be the subgroup with Lie algebra

$$
\mathfrak{n}_{0}:=\bigoplus_{\substack{\alpha \in \Sigma^{+} \\ \alpha \notin\left\{\varepsilon_{1}-\varepsilon_{2}, \varepsilon_{2}-\varepsilon_{3}\right\}}} \mathfrak{g}^{\alpha} .
$$


Let $Z(M)$ be the center of $M$. Then the proposition shows that $H:=Z(M) A N_{0}$ is a real spherical subgroup. In case $q-p>1$, it is not absolutely spherical.

\section{Degenerations of a real spherical subalgebra}

\subsection{The compression cone}

We recall that the local structure theorem (see [22]) implies that $\mathfrak{g}=\mathfrak{h}+\mathfrak{q}$, and hence

$$
\mathfrak{g}=\mathfrak{h} \oplus(\mathfrak{l} \cap \mathfrak{h})^{\perp \mathfrak{r}} \oplus \mathfrak{u} .
$$

Here we use the Cartan-Killing form of $\mathfrak{g}$ restricted to $\mathfrak{l}$ to define $\perp_{\mathfrak{l}}$. Note that since $\mathfrak{l} \cap \mathfrak{h}$ is $\operatorname{Ad}(A)$-invariant, then so is its orthocomplement. In particular, the decomposition (3.1) will not be affected by our later conjugation of the Cartan involution by an element from $A$.

We define the linear operator $T: \overline{\mathfrak{u}} \rightarrow(\mathfrak{l} \cap \mathfrak{h})^{\perp_{\mathfrak{l}}} \oplus \mathfrak{u} \subset \mathfrak{p}_{\text {min }}$ as minus the restriction of the projection along $\mathfrak{h}$, according to (3.1). Then

$$
\mathfrak{h}=\mathfrak{l} \cap \mathfrak{h} \oplus \mathcal{G}(T)=\mathfrak{l} \mathfrak{h} \oplus\{\bar{X}+T(\bar{X}): \bar{X} \in \overline{\mathfrak{u}}\} .
$$

Write $\Sigma_{\mathfrak{u}}$ for the space of $\mathfrak{a}$-weights of the $\mathfrak{a}$-module $\mathfrak{u}$. Let $\alpha \in \Sigma_{\mathfrak{u}}$ and let $X_{-\alpha} \in$ $\mathfrak{g}^{-\alpha} \subset \overline{\mathfrak{u}}$. Then

$$
T\left(X_{-\alpha}\right)=\sum_{\beta \in \Sigma_{u} \cup\{0\}} X_{\alpha, \beta},
$$

where $X_{\alpha, \beta} \in \mathfrak{g}^{\beta} \subset \mathfrak{u}$ is a root vector for $\beta \neq 0$, with the convention that $X_{\alpha, 0} \in(\mathfrak{l} \cap \mathfrak{h})^{\perp_{\mathfrak{l}}}$. Let $\mathcal{M} \subset \mathbb{N}_{0}\left[\Sigma_{\mathfrak{u}}\right]$ be the monoid (additive semi-group with zero) generated by

$$
\left\{\alpha+\beta: \alpha \in \Sigma_{\mathfrak{u}}, \beta \in \Sigma_{\mathfrak{u}} \cup\{0\} \text { with } X_{\alpha, \beta} \neq 0 \text { for some } X_{-\alpha} \in \mathfrak{g}^{-\alpha}\right\} .
$$

Note that elements of $\mathcal{M}$ vanish on $\mathfrak{a}_{H}$, so that $\mathcal{M}$ is naturally a subset of $\mathfrak{a}_{Z}^{*}$.

We define the compression cone of $Z$ to be

$$
\mathfrak{a}_{Z}^{-}:=\left\{X \in \mathfrak{a}_{Z}: \alpha(X) \leqslant 0 \text { for all } \alpha \in \mathcal{M}\right\},
$$

which is a closed convex cone in $\mathfrak{a}_{Z}$ with non-empty interior.

\subsubsection{Limits in the Grassmannian}

We recall from [20, Lemma 5.9] the following property of the compression cone. Let $\mathfrak{a}_{Z}^{--}$ be the interior of $\mathfrak{a}_{Z}^{-}$, and let

$$
\mathfrak{h}_{\lim }:=\mathfrak{l} \cap \mathfrak{h}+\overline{\mathfrak{u}}
$$


Note that $d:=\operatorname{dim} \mathfrak{h}=\operatorname{dim} \mathfrak{h}_{\lim }$ and that $\mathfrak{h}_{\lim }$ is a real spherical subalgebra. Then $X \in \mathfrak{a}_{Z}^{--}$ if and only if

$$
\lim _{t \rightarrow \infty} e^{t \operatorname{ad} X} \mathfrak{h}=\mathfrak{h}_{\text {lim }}
$$

holds in the Grassmannian $\operatorname{Gr}_{d}(\mathfrak{g})$ of $d$-dimensional subspaces of $\mathfrak{g}$.

\subsubsection{Limits in a representation}

We recall another description of $\mathfrak{a}_{Z}^{-}$, which was used as its definition in [20, Definition 5.1 and Lemma 5.10].

Consider an irreducible finite-dimensional real representation $(\pi, V)$ with $H$-semispherical vector $0 \neq v_{H} \in V$, that is there is an algebraic character $\chi$ of $H$ such that $\pi(h) v_{H}=\chi(h) v_{H}$ for all $h \in H$. Let $\mathbb{R}^{+} v_{0}$ be a lowest weight ray which is stabilized by $\bar{Q}$. Let $\mathfrak{a}_{\pi, \chi}^{--}$be the open cone in $\mathfrak{a}_{Z}$ defined by the following property: $X \in \mathfrak{a}_{\pi, \chi}^{--}$if and only if

$$
\lim _{t \rightarrow \infty}\left[\pi(\exp (t X)) \cdot v_{H}\right]=\left[v_{0}\right]
$$

holds in the projective space $\mathbb{P}(V)$. We denote by $\mathfrak{a}_{\pi, \chi}^{-}$the closure of $\mathfrak{a}_{\pi, \chi}^{--}$and record that $\mathfrak{a}_{Z}^{-} \subset \mathfrak{a}_{\pi, \chi}^{-}$. Moreover, we have

$$
\mathfrak{a}_{Z}^{-}=\mathfrak{a}_{\pi, \chi}^{-} \text {if and only if } \pi \text { is regular. }
$$

Here $\pi$ is called regular if $\bar{Q}$ is the stabilizer of $\mathbb{R}^{+} v_{0}$; in particular, the lowest weight is strictly anti-dominant with respect to the roots of $\mathfrak{u}$.

\subsection{Spherical roots}

Let $C$ be the convex cone spanned by $\mathcal{M}$. Then, according to [17, Corollaries 12.5 and 10.9], $C$ is simplicial, i.e., there exists a linearly independent set $S \subset \mathfrak{a}_{Z}^{*}$ such that

$$
C=\bigoplus_{\sigma \in S} \mathbb{R}_{\geqslant 0} \sigma
$$

In particular, we record

$$
\mathfrak{a}_{Z}^{-}=\left\{X \in \mathfrak{a}_{Z}: \sigma(X) \leqslant 0 \text { for all } \sigma \in S\right\} .
$$

The elements of $S$, suitably normalized (see [35] for an overview on some commonly used normalizations), are referred to as spherical roots for $Z$. In this paper we are not very specific about the normalization of $S$ and just request that

$$
\mathcal{M} \subset \mathbb{N}_{0}[S]
$$


is satisfied. We note that, by [17, Theorem 11.6], there exists such a normalization of $S$; see [17, equation (11.4)]. We also note that (3.7) implies

$$
S \subset \mathbb{Q} \geqslant 0\left[\Sigma_{\mathfrak{u}}\right]
$$

To see this, let $\sigma \in S$. Then the ray $\mathbb{R}_{\geqslant 0} \sigma$ is extreme in $C$, hence spanned by some $\gamma \in \mathcal{M}$. Thus $\sigma=c \gamma$ for some $c>0$. It now follows from (3.7) that $1 / c$ is an integer, and hence (3.8) holds.

By slight abuse of common terminology, we will henceforth call any set $S$ which satisfies (3.6) and (3.7) a set of spherical roots for $Z$. Let us now fix such a choice.

Given a closed convex cone $C$ in a finite-dimensional real vector space, we call $E(C):=C \cap(-C)$ the edge of $C$; it is the largest vector subspace of $V$ which is contained in $C$.

We are now concerned with the edge $\mathfrak{a}_{Z, E}:=E\left(\mathfrak{a}_{Z}^{-}\right)$of $\mathfrak{a}_{Z}^{-}$. By our definition of $\mathfrak{a}_{Z}^{-}$, we have

$$
\mathfrak{a}_{Z, E}=\left\{X \in \mathfrak{a}_{Z}: \alpha(X)=0 \text { for all } \alpha \in S\right\} .
$$

It is immediate from (3.3) that $\mathfrak{a}_{Z, E}$ is contained in $N_{\mathfrak{g}}(\mathfrak{h})$, the normalizer of $\mathfrak{h}$ in $\mathfrak{g}$. In this context, it is good to keep in mind that $N_{G}(\mathfrak{h}) / H A_{Z, E}$ is a compact group (see [20]).

Let $e:=\operatorname{dim} \mathfrak{a}_{Z, E}, r:=\operatorname{rank}_{\mathbb{R}}(Z)=\operatorname{dim} \mathfrak{a}_{Z}$ and $s:=\# S$. Then $\mathfrak{a}_{Z}^{-} / \mathfrak{a}_{Z, E}$ is a simplicial cone with $s=r-e$ generators.

Example 3.1. Let $H=\bar{N}$. This is a spherical subgroup. In this case $\mathfrak{h}_{\lim }=\mathfrak{h}, \mathcal{M}=\{0\}$, $S=\varnothing$, and $\mathfrak{a}_{Z}^{-}=\mathfrak{a}_{Z, E}=\mathfrak{a}$.

\subsection{Boundary degenerations}

For each subset $I \subset S$ we choose an element $X=X_{I} \in \mathfrak{a}_{Z}^{-}$with $\alpha(X)=0$ for all $\alpha \in I$ and $\alpha(X)<0$ for all $\alpha \in S \backslash I$. Then we define

$$
\mathfrak{h}_{I}:=\lim _{t \rightarrow \infty} e^{\operatorname{ad} t X} \mathfrak{h}
$$

with the limit taken in the Grassmannian $\operatorname{Gr}_{d}(\mathfrak{g})$ as in (3.4). In particular, $\mathfrak{h}_{\varnothing}=\mathfrak{h}_{\text {lim }}$ and $\mathfrak{h}_{S}=\mathfrak{h}$.

To see that the limit exists, we recall the explicit description of $\mathfrak{h}$ in (3.3). Let $\langle I\rangle \subset$ $\mathbb{N}_{0}[S]$ be the monoid generated by $I$. Within the notation of (3.3), we set $X_{\alpha, \beta}^{I}:=X_{\alpha, \beta}$, if $\alpha+\beta \in\langle I\rangle$, and zero otherwise. Let $\mathfrak{u}_{I} \subset \mathfrak{u}$ be the subspace spanned by all $X_{\alpha, \beta}^{I}$, and define a linear operator

$$
T_{I}: \overline{\mathfrak{u}} \longrightarrow(\mathfrak{l} \cap \mathfrak{h})^{\perp_{\mathfrak{l}}} \oplus \mathfrak{u}_{I}
$$


by

$$
T_{I}\left(X_{-\alpha}\right)=\sum_{\beta \in \Sigma_{\mathfrak{u}} \cup\{0\}} X_{\alpha, \beta}^{I} .
$$

In particular, $T_{\varnothing}=0$ and $T_{S}=T$. Now observe that

$$
e^{t \operatorname{ad} X}\left(X_{-\alpha}+T\left(X_{-\alpha}\right)\right)=e^{-t \alpha(X)}\left(X_{-\alpha}+\sum_{\beta} e^{t(\alpha(X)+\beta(X))} X_{\alpha, \beta}\right),
$$

from which we infer that the limit in (3.9) is given by

$$
\mathfrak{h}_{I}=\mathfrak{l} \cap \mathfrak{h}+\mathcal{G}\left(T_{I}\right)=\mathfrak{l} \cap \mathfrak{h}+\left\{\bar{X}+T_{I}(\bar{X}): \bar{X} \in \overline{\mathfrak{u}}\right\}
$$

and, in particular, it is thus independent of the choice of the element $X_{I}$.

Let $H_{I}<G$ be the connected subgroup of $G$ corresponding to $\mathfrak{h}_{I}$. We call $Z_{I}:=G / H_{I}$ the boundary degeneration of $Z$ attached to $I \subset S$, and summarize its basic properties as follows.

Proposition 3.2. Let $I \subset S$. Then

(1) $Z_{I}$ is a real spherical space;

(2) $Q$ is a $Z_{I}$-adapted parabolic subgroup;

(3) $\mathfrak{a} \cap \mathfrak{h}_{I}=\mathfrak{a} \cap \mathfrak{h}$ and $\operatorname{rank}_{\mathbb{R}} Z_{I}=\operatorname{rank}_{\mathbb{R}} Z$;

(4) $I$ is a set of spherical roots for $Z_{I}$;

(5) $\mathfrak{a}_{Z_{I}}=\mathfrak{a}_{Z}$ and $\mathfrak{a}_{Z_{I}}^{-}=\left\{X \in \mathfrak{a}_{Z}: \alpha(X) \leqslant 0\right.$ for all $\left.\alpha \in I\right\}$.

Proof. It follows from (3.9) that $\mathfrak{h}_{I}$ is algebraic, and from (3.12) that $\mathfrak{h}_{I}+\mathfrak{p}_{\min }=\mathfrak{g}$. Thus (1) holds. Statements (2)-(4) all follow easily from (3.12), and (5) is a consequence of (3) and (4).

The boundary degeneration $Z_{I}$ admits non-trivial automorphisms when $I \neq S$. Set

$$
\mathfrak{a}_{I}:=\left\{X \in \mathfrak{a}_{Z}: \alpha(X)=0 \text { for all } \alpha \in I\right\} .
$$

Then we see that $A_{I}$ acts by $G$-automorphisms of $Z_{I}$ from the right.

In the sequel we realize $\mathfrak{a}_{Z}$ as a subspace of $\mathfrak{a}$ via the identification $\mathfrak{a}_{Z}=\mathfrak{a}_{H}^{\perp}$. Likewise we view $A_{Z}$ as a subgroup of $A$.

It is then immediate from the definitions that

$$
\mathfrak{a}_{S}=\mathfrak{a}_{Z, E} \subset \mathfrak{a}_{I}=\mathfrak{a}_{Z_{I}, E} \subset \mathfrak{a}_{\varnothing}=\mathfrak{a}_{Z}
$$

and

$$
\left[\mathfrak{a}_{I}+\mathfrak{a}_{H}, \mathfrak{h}_{I}\right] \subset \mathfrak{h}_{I} .
$$


Remark 3.3. If $Z$ is absolutely spherical, then so are all the $Z_{I}$ 's. Indeed, let $(\mathfrak{g}, \mathfrak{h})$ be absolutely spherical with complex spherical complexification $\left(\mathfrak{g}_{\mathbb{C}}, \mathfrak{h}_{\mathbb{C}}\right)$. Then the compression cones for $(\mathfrak{g}, \mathfrak{h})$ and $\left(\mathfrak{g}_{\mathbb{C}}, \mathfrak{h}_{\mathbb{C}}\right)$ are compatible (see [17, Proposition 5.5 (ii)]) in the obvious sense. From that the assertion follows.

Example 3.4. If $G / H$ is a symmetric space for an involution $\sigma$, that is, $H$ is the connected component of the fixed-point group of an involution $\tau$ on $G$ (which we may assume commutes with $\theta$ ), then

$$
Z_{I}=G /\left(L_{I} \cap H\right)_{e} N_{I},
$$

where $P_{I}=L_{I} N_{I}$ is a $\tau \theta$-stable parabolic subgroup with $\tau$ - and $\theta$-stable Levi part $L_{I}$.

\subsection{Polar decomposition}

The compression cone $\mathfrak{a}_{Z}^{-}$of $Z$ determines the large scale behaviour of $Z$. In [20] we obtained a polar decomposition of a real spherical space. Our concern here is to obtain polar decompositions for all spaces $Z_{I}$ in a uniform way. For that, it is more convenient to use standard compactifications of $Z$ (see [17]), rather then the simple compactifications from $[20]$.

For a real spherical subalgebra $\mathfrak{h}<\mathfrak{g}$ we set $\hat{\mathfrak{h}}:=\mathfrak{h}+\mathfrak{a}_{Z, E}$. Note that $\mathfrak{h} \triangleleft \hat{\mathfrak{h}}$ is an ideal. We denote by $\widehat{H}_{\mathbb{C}, 0}$ the connected algebraic subgroup of $G_{\mathbb{C}}$ with Lie algebra $\hat{\mathfrak{h}}_{\mathbb{C}}$ and set $\widehat{H}_{0}:=\widehat{H}_{\mathbb{C}, 0} \cap G$. More generally, let $\widehat{H}_{\mathbb{C}}$ be some complex algebraic subgroup of $G_{\mathbb{C}}$ with Lie algebra $\hat{\mathfrak{h}}_{\mathbb{C}}$, and let $\widehat{H}=G \cap \widehat{H}_{\mathbb{C}}$. Then $\widehat{H}_{0}$ and $\widehat{H}$ both have Lie algebra $\hat{\mathfrak{h}}$, and $\widehat{H}_{0} \triangleleft \widehat{H}$ is a normal subgroup.

Further, we set $\hat{\mathfrak{h}}_{I}=\mathfrak{h}_{I}+\mathfrak{a}_{I}$ for each $I \subset S$, and note that $\hat{\mathfrak{h}}_{S}=\hat{\mathfrak{h}}$ and $\hat{\mathfrak{h}}_{\varnothing}=\mathfrak{h}_{\text {lim }}+\mathfrak{a}_{Z}$. Recall the element $X_{I} \in \mathfrak{a}_{I} \cap \mathfrak{a}_{Z}^{-}$, and set for $s \in \mathbb{R}$

$$
a_{s, I}:=\exp \left(s X_{I}\right) \in A_{I} .
$$

Let $\widehat{Z}=G / \widehat{H}$. We first describe the basic structure of a standard compactification $\bar{Z}$ for $\widehat{Z}$. There exists a finite-dimensional real representation $V$ of $G$ with an $\widehat{H}$-fixed vector $v_{\widehat{H}}$ such that

$$
\begin{array}{r}
\widehat{Z} \longrightarrow \mathbb{P}(V), \\
g \cdot \hat{z}_{0} \longmapsto\left[g \cdot v_{\widehat{H}}\right],
\end{array}
$$

is an embedding and $\bar{Z}$ is the closure of $\widehat{Z}$ in the projective space $\mathbb{P}(V)$. Moreover, $\bar{Z}$ has the following properties:

(i) the limit $\hat{z}_{0, I}=\lim _{s \rightarrow \infty} a_{s, I} \cdot \hat{z}_{0}$ exists for every $I \subset S$, and the stabilizer $\widehat{H}_{I}$ of $\hat{z}_{0, I}$ is an algebraic group with Lie algebra $\hat{\mathfrak{h}}_{I}$;

(ii) $\bar{Z}$ contains the unique closed orbit $Y=G \cdot \hat{z}_{0, \varnothing}$. 
Note that $\widehat{H}_{I} \supset \widehat{H}_{I, 0}$. The inclusion can be proper, also if we choose $\widehat{H}=\widehat{H}_{0}$. This is the reason why in the first place we need to consider algebraic subgroups $\widehat{H}$ more general than $\widehat{H}_{0}$.

In the next step we explain the polar decomposition for $\widehat{Z}$ and derive from that a polar decomposition for $Z$.

In order to do that we recall the description of the open $P_{\min } \times \widehat{H}$ double cosets of $G$ from $[20, \S 2.4]$. We first treat the case of $\widehat{H}_{0}$. Every open double coset of $P_{\min } \times \widehat{H}_{0}$ has a representative of the form

$$
w=t h, \quad t \in T_{Z}=\exp \left(i \mathfrak{a}_{Z}\right) \text { and } h \in \widehat{H}_{\mathbb{C}, 0} .
$$

This presentation is unique in the sense that if $t^{\prime} h^{\prime}$ is another such representative of the same double coset, then there exist $f \in T_{Z} \cap \widehat{H}_{\mathbb{C}, 0}$ and $h^{\prime \prime} \in \widehat{H}_{0}$ such that $t^{\prime}=t f$ and $h^{\prime}=f^{-1} h h^{\prime \prime}$. We let

$$
\mathcal{F}=\left\{w_{1}, \ldots, w_{k}\right\} \subset G
$$

be a minimal set of representatives of the open $P_{\min } \times \widehat{H}_{0}$-cosets which are of the form (3.15).

The map $w \mapsto P_{\min } w \widehat{H}$ is surjective from $\mathcal{F}$ onto the set of open $P_{\min } \times \widehat{H}$ cosets in $G$. We let

$$
\widehat{\mathcal{F}}=\left\{\widehat{w}_{1}, \ldots, \widehat{w}_{m}\right\} \subset \mathcal{F}
$$

be a minimal set of representatives of these cosets. Note that every $\widehat{w} \in \widehat{\mathcal{F}}$ allows a presentation $\widehat{w}=t h$ as in (3.15), which is then unique in the sense that if $t^{\prime} h^{\prime} \in P_{\min } \widehat{w} \widehat{H}$ is another such representative, then $t^{\prime}=t f$ and $h^{\prime}=f^{-1} h h^{\prime \prime}$ for some $f \in T_{Z} \cap \widehat{H}_{\mathbb{C}}$ and $h^{\prime \prime} \in \widehat{H}$. We observe the following relations on the $T_{Z}$-parts:

$$
\left\{\hat{t}_{1}, \ldots, \hat{t}_{m}\right\}\left(T_{Z} \cap \widehat{H}_{\mathbb{C}}\right)=\left\{t_{1}, \ldots, t_{k}\right\}\left(T_{Z} \cap \widehat{H}_{\mathbb{C}}\right)
$$

With that notation, the polar decomposition for $\widehat{Z}=G / \widehat{H}$ is obtained as in [20, Theorem 5.13]:

$$
\widehat{Z}=\Omega A_{Z}^{-} \widehat{\mathcal{F}} \cdot \hat{z_{0}} \text {. }
$$

Here $A_{Z}^{-}=\exp \left(\mathfrak{a}_{Z}^{-}\right)$, and $\Omega \subset G$ is a compact subset which is of the form $\Omega=\mathcal{F}^{\prime \prime} K$ with $\mathcal{F}^{\prime \prime} \subset G$ a finite set.

For $G / \widehat{H}_{0}$ the polar decomposition (3.16) can be rephrased as $G=\Omega A_{Z}^{-} \mathcal{F} \widehat{H}_{0}$. From the fact that $\widehat{H}_{\mathbb{C}, 0}$ is connected, we infer

$$
\widehat{H}_{0}<N_{G}(H) .
$$


Let $\mathcal{F}^{\prime} \subset \widehat{H}_{0}$ be a minimal set of representatives of the finite group $\widehat{H}_{0} / H A_{Z, E}$ (observe that $H A_{Z, E}$ is the identity component of $\widehat{H}_{0}$ ). Note that $\mathcal{F}^{\prime}$ is in the normalizer of $H$ by (3.17). We then record the obvious decomposition

$$
\widehat{H}_{0}=A_{Z, E} \mathcal{F}^{\prime} H
$$

Note that, since $A_{Z, E}$ is connected, the open $P_{\min } \times H$ double cosets in $G$ are identical to the open $P_{\min } \times A_{Z, E} H$ double cosets. Hence $\mathcal{W}:=\mathcal{F} \mathcal{F}^{\prime} \subset G$ is a (not necessarily minimal) set of representatives for all open $P_{\min } \times H$-double cosets in $G$.

The next lemma guarantees that we can slide $A_{Z, E}$ past $\mathcal{W}$.

Lemma 3.5. Let $w \in \mathcal{W}$. Then there exist for all $a \in A_{Z, E}$ an element $h_{a} \in H$ such that $a^{-1} w a=w h_{a}$. In particular, $\mathcal{W} A_{Z, E} \subset A_{Z, E} \mathcal{W} H$.

Proof. Let $w=t h \in \mathcal{W}$, with $t \in T_{Z}$ and $h \in \widehat{H}_{\mathbb{C}, 0}$. For $a \in A_{Z, E}$ the element $a^{-1} w a$ represents the same open double $P_{\min } \times \widehat{H}_{0}$ coset as $w$. Further note that $a^{-1} w a=t a^{-1} h a$. We infer from the uniqueness of the presentation $w=t h$ (as a representative of an open $P_{\min } \times \widehat{H}_{0}$ double coset) that there exists an element $h_{a} \in \widehat{H}_{0}$ such that $a^{-1} h a=h h_{a}$. Note that $\widehat{H}_{\mathbb{C}, 0}=A_{Z, E, \mathbb{C}} H_{\mathbb{C}}$, and therefore we can decompose $h=b h_{1}$, with $b \in A_{Z, E, \mathbb{C}}$ and $h_{1} \in H_{\mathbb{C}}$. It follows that $a^{-1} h_{1} a=h_{1} h_{a}$. Hence $h_{a} \in \widehat{H}_{0} \cap H_{\mathbb{C}}$ and, as $h_{a}$ varies continuously with $a$, we deduce that it belongs to $H$.

Observe that $A_{Z, E} \subset A_{Z}^{-}$. Thus, putting (3.16), (3.18) and Lemma 3.5 together, we arrive at the following polar decomposition for $Z$ :

$$
Z=\Omega A_{Z}^{-} \mathcal{W} \cdot z_{0}
$$

Remark 3.6. Consider the case where $Z=G / H$ is a symmetric space as in Example 3.4. We choose $\mathfrak{a}$ such that it is $\tau$-stable and such that the (-1)-eigenspace $\mathfrak{a}_{p q}$ of $\tau$ on $\mathfrak{a}$ is maximal. Then $\mathfrak{a}_{Z}=\mathfrak{a}_{H}^{\perp}=\mathfrak{a}_{p q}$ and the set of $P_{\min } \times H$ open double cosets is naturally identified with the quotient of Weyl groups $W_{p q} / W_{H \cap K}$, where $W_{p q}=N_{K}\left(\mathfrak{a}_{p q}\right) / Z_{K}\left(\mathfrak{a}_{p q}\right)$ and $W_{H \cap K}=N_{H \cap K}\left(\mathfrak{a}_{p q}\right) / Z_{H \cap K}\left(\mathfrak{a}_{p q}\right)$ (see [33, Corollary 17]). Moreover, in this case (3.19) is valid with $\Omega=K$ (see [14, Theorem 4.1]).

For $g \in G$ we set $\mathfrak{h}_{g}:=\operatorname{Ad}(g) \mathfrak{h}$ and $H_{g}=g H g^{-1}$, and note that if $P_{\min } g H$ is open then $Z_{g}=G / H_{g}$ is a real spherical space. In particular this applies when $g \in \mathcal{W}$.

LEMmA 3.7. Let $w=t h \in \mathcal{W}$, with $t \in T_{Z}$ and $h \in \widehat{H}_{\mathbb{C}, 0}$. Then

$$
\mathfrak{h}_{w}=\mathfrak{l} \cap \mathfrak{h}+\mathcal{G}\left(T_{w}\right) \text {, }
$$

where $T_{w}: \overline{\mathfrak{u}} \rightarrow \mathfrak{u}+(\mathfrak{l} \cap \mathfrak{h})^{\perp}$ is a linear map with

$$
T_{w}\left(X_{-\alpha}\right)=\sum_{\beta} \varepsilon_{\alpha, \beta}(w) X_{\alpha, \beta}
$$

in the notation from (3.3), and where $\varepsilon_{\alpha, \beta}(w)=t^{\alpha+\beta} \in\{-1,1\}$. 
Proof. We first observe that

$$
\mathfrak{h}_{w}=\operatorname{Ad}(t) \mathfrak{h}_{\mathbb{C}} \cap \mathfrak{g} .
$$

Now, by (3.2), an arbitrary element $X \in \mathfrak{h}_{\mathbb{C}}$ can be uniquely written as

$$
X=X_{0}+\sum_{\alpha \in \Sigma_{\mathfrak{u}}} c_{\alpha}\left(X_{-\alpha}+\sum_{\beta \in \Sigma_{\mathfrak{u}} \cup\{0\}} X_{\alpha, \beta}\right),
$$

with $X_{0} \in(\mathfrak{l} \cap \mathfrak{h})_{\mathbb{C}}$ and with coefficients $c_{\alpha} \in \mathbb{C}$. Hence

$$
\operatorname{Ad}(t) X=X_{0}+\sum_{\alpha \in \Sigma_{\mathfrak{u}}} t^{-\alpha} c_{\alpha}\left(X_{-\alpha}+\sum_{\beta \in \Sigma_{\mathfrak{u}} \cup\{0\}} t^{\alpha+\beta} X_{\alpha, \beta}\right) .
$$

We conclude that $\operatorname{Ad}(t) X \in \mathfrak{g}$ if and only if $X_{0} \in \mathfrak{l} \cap \mathfrak{h}, c_{\alpha} t^{-\alpha} \in \mathbb{R}$ for all $\alpha$ and $t^{\alpha+\beta} \in \mathbb{R}$ for all $\alpha$ and $\beta$, that is, $t^{\alpha+\beta} \in\{-1,1\}$.

Corollary 3.8. Let $w \in \mathcal{W}$. Then

(1) $Q$ is the $Z_{w}$-adapted parabolic subgroup;

(2) $\mathfrak{a}_{Z}^{-}$is the compression cone for $Z_{w}$.

Proof. Immediate from Lemma 3.7.

\subsubsection{The sets $\mathcal{F}$ and $\mathcal{W}$ for the boundary degenerations}

Let $I \subset S$. We define $\widehat{H}_{I}$ as in (i), with the assumption $\widehat{H}=\widehat{H}_{0}$, and set

$$
\widehat{Z}_{I}:=G / \widehat{H}_{I}=G \cdot \hat{z}_{0, I} \cdot
$$

We wish to construct a set $\mathcal{F}_{I}$ of representatives of open $P_{\min } \times \widehat{H}_{I}$ double cosets in $G$, analogous to the previous set $\mathcal{F}$ for $P_{\min } \times \widehat{H}_{0}$. Recall that possibly $\widehat{H}_{I, 0} \varsubsetneqq \widehat{H}_{I}$.

Notice that the $\widehat{Z}_{I}$-adapted parabolic subgroup is $Q$ and that $L L_{\mathrm{n}} \subset L \cap \widehat{H}_{I}$. The local structure theorem for $\widehat{Z}_{I}$ then implies that $Q \times{ }_{L}\left(L / L \cap \widehat{H}_{I}\right) \rightarrow \widehat{Z}_{I}$ is an open immersion onto the $P_{\min }$-orbit $P_{\min } \cdot \hat{z}_{0, I}$. Moreover $A_{Z_{I}}=A_{Z} / A_{I}$. Realize $\mathfrak{a}_{Z, I} \subset \mathfrak{a}_{Z}$ via $\mathfrak{a}_{I}^{\perp_{\mathfrak{a}_{Z}}}$ and set $T_{Z_{I}}=\exp \left(i \mathfrak{a}_{Z_{I}}\right)$. Let $\widehat{\mathcal{F}}_{I}$ be a minimal set of representatives of the open $P_{\min } \times \widehat{H}_{I}$ double cosets which are of the form $\widehat{w}_{I}=\hat{t}_{I} \hat{h}_{I} \in \widehat{\mathcal{F}}_{I}$, with $\hat{t}_{I} \in T_{Z_{I}}$ and $\hat{h}_{I} \in \widehat{H}_{I, \mathbb{C}}$. As before, we also have a minimal set $\mathcal{F}_{I}$ of representatives for the open cosets for the smaller group $P_{\min } \times \widehat{H}_{I, 0}$, such that

$$
\widehat{\mathcal{F}}_{I} \subset \mathcal{F}_{I}=\left\{w_{1, I}, \ldots, w_{k_{I}, I}\right\},
$$

where $w_{j, I}=t_{j, I} h_{j, I}$ with $t_{j, I} \in T_{Z_{I}}$ and $h_{j, I} \in \widehat{H}_{I, \mathbb{C}, 0}$.

Finally, in analogy to $\mathcal{F}^{\prime}$, we choose $\mathcal{F}_{I}^{\prime}$ as a minimal set of representatives for $\widehat{H}_{I, 0} / H_{I} A_{I}$, and set $\mathcal{W}_{I}:=\mathcal{F}_{I} \mathcal{F}_{I}^{\prime}$. Then the polar decomposition of $Z_{I}$ is given by

$$
Z_{I}=\Omega A_{Z_{I}}^{-} \mathcal{W}_{I} \cdot z_{0, I}
$$

with $\Omega \subset G$ being a compact subset of the form $\mathcal{F}_{I}^{\prime \prime} K$ for a finite set $\mathcal{F}_{I}^{\prime \prime} \subset G$. 


\subsection{Relating $\mathcal{W}_{I}$ to $\mathcal{W}$}

We start with a general lemma.

Lemma 3.9. Let $Z=G / H$ be a real spherical space and $g \in G$ be such that $P_{\min } g H_{I}$ is open in $G$. Then there exists $s_{0}>0$ such that $P_{\min } g a_{s, I} H$ is open and equal to $P_{\min } g a_{s_{0}, I} H$ for all $s \geqslant s_{0}$.

Proof. If there were a sequence $s_{n}>0$ tending to infinity with $\mathfrak{p}+\operatorname{Ad}\left(g a_{s_{n}, I}\right) \mathfrak{h} \mp \mathfrak{g}$ for all $n$, then $\lim _{n \rightarrow \infty}\left(\mathfrak{p}+\operatorname{Ad}(g) \operatorname{Ad}\left(a_{s_{n}, I}\right) \mathfrak{h}\right)=\mathfrak{p}+\operatorname{Ad}(g) \mathfrak{h}_{I}$ would be a subspace of $\mathfrak{g}$ with positive codimension, which contradicts the assumption on $g$. Hence $P_{\min } g a_{s, I} H$ is open for all $s \geqslant s_{0}$, for some $s_{0}$. By continuity, this implies that the sets are equal.

Fix an element $w_{I} \in \mathcal{W}_{I}$ and observe that $P_{\min } w_{I} H_{I}$ is open in $G$. Lemma 3.9 then gives an element $w \in \mathcal{W}$ and an $s_{0}>0$ such that $P_{\min } w_{I} a_{s, I} H=P_{\min } w H$ for all $s \geqslant s_{0}$. We say that $w$ corresponds to $w_{I}$, but note that $w$ is not necessarily unique.

Lemma 3.10. Let $w_{I} \in \mathcal{W}_{I}$ and let $w \in \mathcal{W}$ correspond to $w_{I}$. With $s_{0}>0$ as above, there exist for each $s \geqslant s_{0}$ elements $u_{s} \in U, b_{s} \in A_{Z}, m_{s} \in M$ and $h_{s} \in H$, each depending continuously on $s \geqslant s_{0}$, such that

(1) $w_{I} a_{s, I}=u_{s} b_{s} m_{s} w h_{s}$.

Moreover,

(2) the elements $u_{s}$ and $b_{s}$ are unique and depend analytically on $s$;

(3) $\lim _{s \rightarrow \infty}\left(a_{s, I} b_{s}^{-1}\right)=\mathbf{1}$;

(4) $\lim _{s \rightarrow \infty} u_{s}=\mathbf{1}$

(5) $m_{s}$ can be chosen such that $\lim _{s \rightarrow \infty} m_{s}$ exists in $M$.

Proof. By Corollary 3.8, the map

$$
\begin{aligned}
&\left(U \times A_{Z} \times M\right) /\left(M \cap H_{w}\right) \longrightarrow P_{\min } w \cdot z_{0}, \\
&(u, a, m) \longmapsto u a m w \cdot z_{0},
\end{aligned}
$$

is a diffeomorphism (local structure theorem for $Z_{w}$ ). As $w_{I} a_{s, I} \in P_{\min } w \cdot z_{0}$ for $s \geqslant s_{0}$, this gives (1) and (2).

After enlarging $G$ to $G \times \mathbb{R}^{\times}$we can, via the affine cone construction (see [22, Corollary 3.8]), assume that $Z=G / H$ is quasi-affine.

Let us denote by $\Gamma$ the set (of equivalence classes) of finite-dimensional irreducible $H$-spherical and $K$-spherical representations. To begin with, we recall a few facts from $\S 3.1 .2$ and from [20].

For a representation $(\pi, V) \in \Gamma$ we denote its highest weight by $\lambda_{\pi} \in \mathfrak{a}^{*}$. Let $(\pi, V) \in \Gamma$ and $0 \neq v_{H} \in V$ be an $H$-fixed vector which we expand into a-eigenvectors:

$$
v_{H}=\sum_{\nu \in \Lambda_{\pi}} v_{-\lambda_{\pi}+\nu}
$$


Here $\Lambda_{\pi} \subset \mathbb{N}_{0} \Sigma_{u}$ is such that $-\lambda_{\pi}+\Lambda_{\pi}$ is the $\mathfrak{a}$-weight spectrum of $v_{H}$. Note that $v_{-\lambda_{\pi}}$ is a lowest-weight vector which is fixed by $M$.

As $\left.\Lambda_{\pi}\right|_{\mathfrak{a}_{Z}^{-}} \leqslant 0$ (see [20, Lemma 5.3]), we deduce that the limit

$$
v_{H, I}:=\lim _{s \rightarrow \infty} a_{s, I}^{\lambda_{\pi}} \pi\left(a_{s, I}\right) v_{H}
$$

exists. Moreover, with $\Lambda_{\pi, I}:=\left\{\nu \in \Lambda_{\pi}: \nu\left(X_{I}\right)=0\right\}$, we obtain that $v_{H, I}=\sum_{\nu \in \Lambda_{\pi, I}} v_{-\lambda_{\pi}+\nu}$. Note that $v_{H, I}$ is $H_{I}$-fixed.

Let $w=t h$ and $w_{I}=t_{I} h_{I}$, with our previous notation. From (1) we obtain

$$
a_{s, I}^{\lambda_{\pi}} \pi\left(t_{I} h_{I} a_{s, I}\right) v_{H}=a_{s, I}^{\lambda_{\pi}} \pi\left(u_{s} m_{s} b_{s} t\right) v_{H}
$$

and hence, by passing to the limit $s \rightarrow \infty$,

$$
\pi\left(t_{I}\right) v_{H, I}=\lim _{s \rightarrow \infty} a_{s, I}^{\lambda_{\pi}} \pi\left(u_{s} m_{s} b_{s} t\right) v_{H}
$$

Let $v^{*} \in V^{*}$ be a highest-weight vector in the dual representation, and apply it to (3.23). Since $v^{*}\left(v_{H}\right)=v^{*}\left(v_{H, I}\right)=v^{*}\left(v_{-\lambda_{\pi}}\right) \neq 0$, we get

$$
t_{I}^{-\lambda_{\pi}}=t^{-\lambda_{\pi}} \lim _{s \rightarrow \infty}\left(a_{s, I} b_{s}^{-1}\right)^{\lambda_{\pi}}
$$

and therefore $\lim _{s \rightarrow \infty}\left(a_{s, I} b_{s}^{-1}\right)^{\lambda_{\pi}}=1$. Since $Z$ is quasi-affine, it follows that $\left\{\lambda_{\pi}: \pi \in \Gamma\right\}$ spans $\mathfrak{a}_{Z}^{*}$ (see [22, Lemma 3.4], and hence (3).

We move on to the fourth assertion. We first show that $\left(u_{s}\right)_{s}$ is bounded in $U$ when $s \rightarrow \infty$. For that, let $X_{1}, \ldots, X_{n}$ be a basis for $\mathfrak{u}$ consisting of root vectors $X_{j}$ with associated roots $\alpha_{j}$. The map

$$
\begin{aligned}
\mathbb{R}^{n} & \longrightarrow U \\
\left(x_{1}, \ldots, x_{n}\right) & \longmapsto \exp \left(x_{n} X_{n}\right) \cdot \ldots \cdot \exp \left(x_{1} X_{1}\right),
\end{aligned}
$$

is a diffeomorphism. Let $\left(x_{1}(s), \ldots, x_{n}(s)\right)$ be the coordinate vector of $u_{s} \in U$, which we claim is bounded.

We fix an ordering of $\Sigma_{\mathfrak{u}}$ with the property that if a root $\alpha$ can be expressed as a sum of other roots $\beta$, then only roots $\beta \leqslant \alpha$ will occur. It suffices to show, for any given index $j$, that if $x_{i}(s)$ is bounded for all $i$ with $\alpha_{i}<\alpha_{j}$, then so is $x_{i}(s)$ for each $i$ with $\alpha_{i}=\alpha_{j}$.

We now fix $\pi$ such that it is regular, that is, the highest weight $\lambda=\lambda_{\pi}$ satisfies $\lambda\left(\alpha^{\vee}\right)>0$ for all $\alpha \in \Sigma_{\mathfrak{u}}$. Then the map $X \mapsto d \pi(X) v_{-\lambda}$ is injective from $\mathfrak{u}$ into $V$. 
We compare vectors of weight $-\lambda+\alpha_{j}$ on both sides of (3.23). On the left side we have $t_{I}^{-\lambda+\alpha_{j}} v_{-\lambda+\alpha_{j}}$ if $\alpha_{j} \in \Lambda_{\pi, I}$, and 0 otherwise. By applying the Taylor expansion of exp, we find on the other side

$$
\lim _{s \rightarrow \infty} a_{s, I}^{\lambda}\left(\sum_{m, \nu}\left(b_{s} t\right)^{-\lambda+\nu} \frac{x(s)^{m}}{m !} d \pi\left(X_{n}\right)^{m_{n}} \ldots d \pi\left(X_{1}\right)^{m_{1}} \pi\left(m_{s}\right) v_{-\lambda+\nu}\right),
$$

where the sum extends over all multi-indices $m=\left(m_{1}, \ldots, m_{n}\right)$ and all $\nu \in \Lambda_{\pi}$ for which $\alpha_{j}=\sum_{i=1}^{n} m_{i} \alpha_{i}+\nu$.

Notice that by (3) the product $a_{s, I}^{\lambda}\left(b_{s}\right)^{-\lambda+\nu}=\left(a_{s, I} b_{s}^{-1}\right)^{\lambda-\nu} a_{s, I}^{\nu}$ remains bounded when $s \rightarrow \infty$. Likewise, by our assumption on the index $j$, all the terms with $m_{i} \neq 0$ for some $i$ with $\alpha_{i} \neq \alpha_{j}$ (and hence $m_{i}=0$ for all $i$ with $\alpha_{i}=\alpha_{j}$ ) are bounded. The remaining terms are those of the form

$$
a_{s, I}^{\lambda}\left(b_{s} t\right)^{-\lambda} x_{i}(s) d \pi\left(X_{i}\right) v_{-\lambda}
$$

where $\alpha_{i}=\alpha_{j}$. It follows by linear independence that $x_{i}(s)$ is bounded for each of these $i$ as claimed, i.e. $\left(u_{s}\right)_{s}$ is bounded.

Finally, we show that $u_{s}$ converges to $\mathbf{1}$. Otherwise there exists $u \neq \mathbf{1}$ and a sequence $s_{k}$ of positive numbers tending to infinity such that $u_{k}:=u_{s_{k}} \rightarrow u$. We may assume in addition that $m_{k}:=m_{s_{k}}$ is convergent with a limit $m$. We apply (1) to $\hat{z}_{0} \in \widehat{Z}$ :

$$
w_{I} a_{s_{k}, I} \cdot \hat{z}_{0}=u_{k} m_{k} b_{k} t \cdot \hat{z}_{0}
$$

and take the limit

$$
t_{I} \cdot \hat{z}_{0, I}=u m t \cdot \hat{z}_{0, I}
$$

The local structure theorem for $\widehat{Z}_{I}=G / \widehat{H}_{I}$ then implies $u=\mathbf{1}$, which completes the proof of (4).

The proof of (4) shows as well that the limit $m$ of every converging subsequence of $m_{s}$ satisfies $t_{I} \cdot \hat{z}_{0, I}=m t \cdot \hat{z}_{0, I}$, and hence determines a unique element in $M /\left(M \cap \widehat{H}_{I}\right)$. Thus $\lim _{s \rightarrow \infty} m_{s}\left(M \cap \widehat{H}_{I}\right)$ exists. Notice that $\left(M \cap H_{w}\right)_{e}$ has finite index in $M \cap H_{I}$, as the Lie algebras of the two groups coincide. By continuity with respect to $s$, it follows that $m_{s}\left(M \cap H_{w}\right)_{e}$ converges in $M /\left(M \cap H_{w}\right)_{e}$. Now (5) follows by trivializing this bundle in a neighborhood of the limit point.

Remark 3.11. With the assumption and notation of the preceding lemma, let $m:=$ $\lim _{s \rightarrow \infty} m_{s}$. Then (3.24) implies the relation

$$
\left(\hat{\mathfrak{h}}_{I}\right)_{w_{I}}=\operatorname{Ad}(m)\left(\hat{\mathfrak{h}}_{w}\right)_{I},
$$


where $\left(\hat{\mathfrak{h}}_{w}\right)_{I}:=\left(\mathfrak{h}_{w}\right)_{I}+\mathfrak{a}_{I}$. To see this, first note that $\mathfrak{a}_{I} \subset\left(\hat{\mathfrak{h}}_{I}\right)_{w_{I}}$ by Lemma 3.5, and hence (by dimension) it suffices to show that

$$
\operatorname{Ad}(m)\left(\mathfrak{h}_{w}\right)_{I} \subset\left(\hat{\mathfrak{h}}_{I}\right)_{w_{I}}
$$

Let $X \in\left(\mathfrak{h}_{w}\right)_{I}$ and choose $X(s) \in \operatorname{Ad}\left(a_{s, I}\right) \mathfrak{h}_{w}$ with $X(s) \rightarrow X$ for $s \rightarrow \infty$. The fundamental vector field on $\widehat{Z}_{w}$ corresponding to $\operatorname{Ad}(m) X(s)$ has a zero at $m a_{s, I} w \cdot \hat{z}_{0}$, and from

$$
t \cdot \hat{z}_{0, I}=t \cdot \lim _{s \rightarrow \infty} a_{s, I} \cdot \hat{z}_{0}=\lim _{s \rightarrow \infty} a_{s, I} w \cdot \hat{z}_{0}
$$

we deduce that the fundamental vector field corresponding to $\operatorname{Ad}(m) X$ then has a zero at $m t \cdot \hat{z}_{0, I}$. Now, (3.25) follows from (3.24) and the fact that $\left(\widehat{H}_{I}\right)_{w_{I}}$ is the stabilizer of $t_{I} \cdot \hat{z}_{0, I}=w_{I} \cdot \hat{z}_{0, I}$.

\subsection{Unimodularity}

For a moment, let $G$ be a an arbitrary Lie group and $H<G$ be a closed subgroup. We call the homogeneous space $Z=G / H$ unimodular provided that $Z$ carries a $G$-invariant positive Borel measure, and recall that this is the case if and only if the attached modular character

$$
\begin{aligned}
\Delta_{Z}: H & \longrightarrow \mathbb{R}, \\
h & \longmapsto \frac{\left|\operatorname{det} \operatorname{Ad}_{\mathfrak{h}}(h)\right|}{\left|\operatorname{det} \operatorname{Ad}_{\mathfrak{g}}(h)\right|}=\left|\operatorname{det} \operatorname{Ad}_{\mathfrak{g} / \mathfrak{h}}(h)\right|^{-1},
\end{aligned}
$$

is trivial.

After these preliminaries, we return to our initial set-up of a real spherical space $Z=G / H$ and its boundary degenerations. In this context, we record the following result.

LEMma 3.12. Let $Z$ be a real spherical space which is unimodular. Then all boundary degenerations $Z_{I}$ are unimodular.

Proof. The fact that the map $X \mapsto \operatorname{tr}(\operatorname{ad} X)$ is trivial in $\mathfrak{h}^{*}$ is a closed condition on $d$-dimensional Lie subalgebras in $\mathfrak{g}$. Now apply (3.9).

\section{Levi-induced spherical spaces}

Let $Z=G / H$ be a real spherical space. Let $P<G$ be a parabolic subgroup and $P=G_{P} U_{P}$ a Levi decomposition. Then $G_{P} \simeq P / U_{P}$. We write

$$
\operatorname{pr}_{P}: P \longrightarrow G_{P}
$$


for the projection homomorphism. Define $H_{P}:=\operatorname{pr}_{P}(H \cap P)$ and set

$$
Z_{P}:=G_{P} / H_{P}
$$

Note that $H_{P}<G_{P}$ is an algebraic subgroup.

Proposition 4.1. The space $Z_{P}$ is real spherical.

Proof. Let $Q_{\min }<P$ be a minimal parabolic subgroup of $G$. According to [26], we have that the number of $Q_{\mathrm{min}}$-orbits in $Z$ is finite. In particular, we have that the number of $Q_{\text {min }}$-orbits in $P /(P \cap H) \subset Z$ is finite. Observe that $Q_{\min , P}:=\operatorname{pr}_{P}\left(Q_{\min }\right)$ is a minimal parabolic subgroup of $G_{P}$. It follows that the number of $Q_{\min , P}$-orbits in $Z_{P}$ is finite. In particular, there exist open orbits, i.e. $Z_{P}$ is real spherical.

We call $Z_{P}$ the Levi-induced real spherical space attached to $P$.

\subsection{Induced parabolics with respect to open $P$-orbits}

In the sequel we are only interested in parabolic subgroups containing the fixed minimal parabolic subgroup $P_{\min }$. We recall the parametrization of these. Recall that $\Sigma=\Sigma(\mathfrak{g}, \mathfrak{a}) \subset \mathfrak{a}^{*}$ is the root system attached to the pair $(\mathfrak{g}, \mathfrak{a})$. Let $\Sigma^{+} \subset \Sigma$ be the positive system attached to $N$, and $\Pi \subset \Sigma^{+}$be the associated set of simple roots. The parabolic subgroups $P \supset P_{\min }$ are in one-to-one correspondence with the subsets $F \subset \Pi$. The parabolic subgroup $P_{F}$ attached to $F \subset \Pi$ has Levi decomposition $P_{F}=G_{F} U_{F}$, where $G_{F}=Z_{G}\left(\mathfrak{a}_{F}\right)$ with

$$
\mathfrak{a}_{F}:=\{X \in \mathfrak{a}: \alpha(X)=0 \text { for all } \alpha \in F\},
$$

and

$$
\mathfrak{u}_{F}:=\bigoplus_{\alpha \in \Sigma^{+} \backslash\langle F\rangle} \mathfrak{g}^{\alpha} .
$$

In these formulas $\mathfrak{g}^{\alpha} \subset \mathfrak{g}$ is the root space attached to $\alpha \in \Sigma$ and $\langle F\rangle \subset \Sigma$ denotes the root system generated by $F$.

The space $\mathfrak{a}$ decomposes orthogonally as $\mathfrak{a}=\mathfrak{a}_{F} \oplus \mathfrak{a}^{F}$, with

$$
\mathfrak{a}^{F}:=\operatorname{span}\left\{\alpha^{\vee}: \alpha \in \Pi \backslash F\right\},
$$

where $\alpha^{\vee} \in \mathfrak{a}$ is the coroot associated with $\alpha$. Observe that $A^{F}$ is a maximal split torus of the semi-simple commutator group $\left[G_{F}, G_{F}\right]$, and that $P_{\min , \mathrm{F}}:=P_{\min } \cap G_{F}$ is a minimal parabolic subgroup of $G_{F}$ with unipotent radical $U^{F}$, where

$$
\mathfrak{u}^{F}:=\bigoplus_{\alpha \in\langle F\rangle^{+}} \mathfrak{g}^{\alpha} .
$$


Denote $\operatorname{pr}_{F}=\operatorname{pr}_{P_{F}}, H_{F}=H_{P_{F}}$ and

$$
Z_{F}:=Z_{P_{F}}=G_{F} / H_{F},
$$

the Levi-induced homogeneous space attached to $P_{F}$.

We write $F_{Q} \subset \Pi$ for the set which corresponds to $Q$. In the sequel we are particularly interested in those parabolic subgroups $P_{F}$ which contain $Q$, that is, for which $F \supset F_{Q}$. For later reference, we note that

$$
\mathfrak{g}^{\alpha} \subset \mathfrak{h}, \quad \alpha \in\left\langle F_{Q}\right\rangle .
$$

\subsection{Examples of induced spaces}

\subsubsection{Symmetric spaces}

Assume, as in Example 3.4, that $Z$ is a symmetric space. The $Z$-adapted parabolic subgroup $Q$ is $\tau \theta$-stable, and so are also all parabolic subgroups $P_{F} \supset Q$. In particular, we have

$$
P_{F} \cap H=G_{F} \cap H=H_{F}
$$

and $Z_{F}=G_{F} / H_{F}$ is a symmetric space, which embeds into $Z$.

\subsubsection{Triple spaces}

For a general real spherical space it is an unfortunate fact that basic properties of $Z$ are typically not inherited by $Z_{F}$. For example, if $Z$ is affine/unimodular/has trivial automorphism group, then one cannot expect the same for the induced space $Z_{F}$. This is all well illustrated in the basic example of triple spaces. Let $\mathcal{G}:=\mathrm{SO}_{e}(1, n)$ for $n \geqslant 2$ and set

$$
G:=\mathcal{G} \times \mathcal{G} \times \mathcal{G}
$$

Then

$$
H:=\Delta_{3}(\mathcal{G}):=\{(g, g, g): g \in \mathcal{G}\}
$$

is a real spherical subgroup of $G$. Let

$$
P_{\text {min }}:=\mathcal{P}_{1} \times \mathcal{P}_{2} \times \mathcal{P}_{3}
$$

be a minimal parabolic subgroup of $G$, that is, each $\mathcal{P}_{i}<\mathcal{G}$ is a minimal (and maximal) parabolic subgroup of $\mathcal{G}$. The condition that $H P_{\min } \subset G$ is open means that all $\mathcal{P}_{i}$ are pairwise different (see [11]). Note that $Q=P_{\min }$ in this case. Note that $\Sigma=A_{1} \times A_{1} \times A_{1}$, 
and thus $\Pi=\left\{\alpha_{1}, \alpha_{2}, \alpha_{3}\right\}$. There are six proper parabolic subgroups $P_{F}$ containing $Q$. For example if $|F|=1$, say $F=\left\{\alpha_{3}\right\}$, one has

$$
P_{\left\{\alpha_{3}\right\}}=\mathcal{P}_{1} \times \mathcal{P}_{2} \times \mathcal{G},
$$

whereas for $|F|=2$, say $F=\left\{\alpha_{2}, \alpha_{3}\right\}$, one has

$$
P_{\left\{\alpha_{2}, \alpha_{3}\right\}}:=\mathcal{P}_{1} \times \mathcal{G} \times \mathcal{G} .
$$

Let $A<P_{\text {min }}$ be a maximal split torus. Then $A=\mathcal{A}_{1} \times \mathcal{A}_{2} \times \mathcal{A}_{3}$. Further, we let $\mathcal{M}_{i}<\mathcal{P}_{i}$ be a maximal compact subgroup which commutes with $\mathcal{A}_{i}$. Denote by $p_{i}: \mathcal{P}_{i} \rightarrow \mathcal{M}_{i} \mathcal{A}_{i}$ the projection along $\mathcal{N}_{i}$. The real spherical subgroups $H_{F}$ for our above choices of $F$ are given by

$$
\begin{aligned}
H_{\left\{\alpha_{3}\right\}} & =\left\{\left(p_{1}(g), p_{2}(g), g\right): g \in \mathcal{P}_{1} \cap \mathcal{P}_{2}\right\} \simeq \mathcal{P}_{1} \cap \mathcal{P}_{2}, \\
H_{\left\{\alpha_{2}, \alpha_{3}\right\}} & =\left\{\left(m_{1} a_{1}, m_{1} a_{1} n_{1}, m_{1} a_{1} n_{1}\right): m_{1} a_{1} n_{1} \in \mathcal{M}_{1} \mathcal{A}_{1} \mathcal{N}_{1}\right\}=\Delta_{3}\left(\mathcal{M}_{1} \mathcal{A}_{1}\right) \Delta_{2}\left(\mathcal{N}_{1}\right) \simeq \mathcal{P}_{1}
\end{aligned}
$$

Of special interest is the case $\mathcal{G}=\operatorname{SO}_{e}(1,2) \simeq \operatorname{PSL}(2, \mathbb{R})$. Here, in the three cases with $|F|=1$, one has that $H_{F}$ is reductive (a split torus), while this is not the case for $|F|=2$. Even more, for $|F|=2$ the spaces $Z_{F}$ are not even unimodular and have nontrivial automorphism groups. We remark that the fine polar geometry of this example is described in [11], and that trilinear functionals related to $Z$ were studied by Bernstein and Reznikov [5].

One might think that there is always a Levi decomposition $P_{F}=G_{F}^{\prime} U_{F}$ for which one has $P_{F} \cap H<G_{F}^{\prime}$. The triple cases with $|F|=2$ show that this is not the case in general. Hence, unlike to the symmetric situation, we cannot expect to have embeddings $Z_{F} \hookrightarrow Z$ in general.

\subsection{Induced adapted parabolics}

For $F \supset F_{Q}$ we let

$$
Q_{F}=Q \cap G_{F}=\operatorname{pr}_{F}(Q),
$$

which is a parabolic subgroup of $G_{F}$. It has the Levi decomposition $Q_{F}=L_{F} U_{Q, F}$, where $L_{F}=L$ and $U_{Q, F}=U \cap G_{F}$.

LEMma 4.2. The following assertions hold:

(1) $Q_{F} H_{F}=P_{\min , \mathrm{F}} H_{F}$ is open in $G_{F}$;

(2) $\mathfrak{l} \cap \mathfrak{h}=\mathfrak{q}_{F} \cap \mathfrak{h}_{F}$;

(3) $Q_{F}$ is the $Z_{F}$-adapted parabolic subgroup of $G_{F}$ containing $P_{\min , F}$. 
Proof. As $Q \subset P_{F}$ and $\operatorname{pr}_{F}: P_{F} \rightarrow G_{F}$ is a homomorphism, we obtain

$$
Q_{F} H_{F}=\operatorname{pr}_{F}\left((Q H) \cap P_{F}\right)=\operatorname{pr}_{F}\left(\left(P_{\min } H\right) \cap P_{F}\right)=P_{\min , \mathrm{F}} H_{F} .
$$

This is an open set, since $\operatorname{pr}_{F}: P_{F} \rightarrow G_{F}$ is an open map. Further we note that the Lie algebra of $Q_{F}$ is given by

$$
\mathfrak{q}_{F}=\mathfrak{q} \cap \mathfrak{g}_{F}=\mathfrak{l}+\mathfrak{u}^{F} .
$$

We recall (3.2). It follows that

$$
\mathfrak{h} \cap \mathfrak{p}_{F}=\mathfrak{l} \cap \mathfrak{h}+\left\{\bar{X}+T(\bar{X}): \bar{X} \in \overline{\mathfrak{u}}^{F}\right\} .
$$

This in turn gives that

$$
\mathfrak{h}_{F}=\operatorname{pr}_{F}\left(\mathfrak{h} \cap \mathfrak{p}_{F}\right)=\mathfrak{l} \cap \mathfrak{h}+\left\{\bar{X}+\left(\operatorname{pr}_{F} \circ T\right)(\bar{X}): \bar{X} \in \overline{\mathfrak{u}}^{F}\right\} .
$$

The combination of (4.2) and (4.3) results in the second assertion. The last assertion now follows, as $L_{F, n}=L_{n} \subset H$ (see $\left.\S 2\right)$.

Observe that the lemma implies that $\mathfrak{a} \cap \mathfrak{h}=\mathfrak{a} \cap \mathfrak{h}_{F}$, and hence that there is an equality of real ranks

$$
\operatorname{rank}_{\mathbb{R}}(Z)=\operatorname{rank}_{\mathbb{R}}\left(Z_{F}\right) .
$$

Furthermore, $A_{Z_{F}}=A_{Z}$.

\subsection{Induced compression cones}

We are interested in the behaviour of the compression cone under induction. Note that there is a natural action of $A$ on $A_{Z}=A / A_{H}$.

Proposition 4.3. Let $F \supset F_{Q}$. Then

$$
A_{F} \cdot A_{Z}^{-}=A_{F} \cdot A_{Z}^{-}
$$

for the induced spherical space $Z_{F}=G_{F} / H_{F}$.

Proof. We shall prove that

$$
A_{Z}^{-} \subset A_{Z_{F}}^{-} \subset A_{F} \cdot A_{Z}^{-} .
$$

Let $(\pi, V)$ be a regular irreducible real $H$-semi-spherical representation as considered in (3.5). This induces a natural $H_{F}$-semi-spherical representation $\left(\pi_{Y}, Y\right)$ of $G_{F}$ as follows. 
Set $Y:=V / \mathfrak{u}_{F} V$. Clearly $Y$ is a $G_{F}$-module. Note that $w_{0}:=v_{0}+\mathfrak{u}_{F} V \in Y$ is a lowest weight vector, and hence generates an irreducible submodule, say $Y_{0}$ of $Y$. As

$$
V=\mathcal{U}(\mathfrak{u}) v_{0}=\mathcal{U}\left(\mathfrak{u}^{F}\right) \mathcal{U}\left(\mathfrak{u}_{F}\right) v_{0} \subset \mathcal{U}\left(\mathfrak{u}^{F}\right)\left(Y_{0}+\mathfrak{u}_{F} V\right),
$$

we conclude that $Y_{0}=Y$ is irreducible. As $\pi$ is regular with respect to $\bar{Q}$, we infer that $w_{0}$ is regular with respect to $\bar{Q}_{F}$. Likewise $w_{H}:=v_{H}+\mathfrak{u}_{F} V$ is an $H_{F}$-semi-spherical vector in $Y$. As

$$
V=\mathcal{U}(\mathfrak{g}) v_{H}=\mathcal{U}(\mathfrak{q}) v_{H},
$$

we conclude that $v_{H} \notin \mathfrak{u} V$. Since $\mathfrak{u}_{F} \subset \mathfrak{u}$, it follows that $w_{H} \neq 0$.

Now, if $X \in \mathfrak{a}_{\pi}^{--}$, then by (3.5)

$$
\lim _{t \rightarrow \infty}\left[\pi_{Y}(\exp (t X)) w_{H}\right]=\left[w_{0}\right] .
$$

This shows the first inclusion in (4.5).

In the construction from above, we realized $Y$ in a quotient of $V$, but it is also possible to realize it as a subspace. Set $\widetilde{Y}:=\mathcal{U}\left(\mathfrak{g}_{F}\right) v_{0}$. Then $\widetilde{Y}$ is an irreducible lowest weight module for $G_{F}$ with lowest weight $v_{0}$, and hence $\widetilde{Y} \simeq Y$. Let us describe an explicit isomorphism. Write $p: V \rightarrow Y$ for the $G_{F}$-equivariant projection. Then the restriction of $\tilde{p}:=\left.p\right|_{\tilde{Y}}$ establishes an isomorphism of $\tilde{p}: \widetilde{Y} \rightarrow Y$. Then $\widetilde{w}_{H}:=\tilde{p}^{-1}\left(w_{H}\right) \in \widetilde{Y}$ is a non-zero $H_{F}$-semi-spherical vector in $\widetilde{Y}$. Then $v_{H}=\widetilde{w}_{H}+\widetilde{w}_{H}^{\perp}$, with $\widetilde{w}_{H}^{\perp} \in \operatorname{ker} p=\mathfrak{u}_{F} V$. Let now $X \in \mathfrak{a}_{Z_{F}}^{-}$. By adding a suitable element $X^{\prime} \in \mathfrak{a}_{F}$ to $X$, we obtain that $\alpha\left(X+X^{\prime}\right)<0$ for all roots $\alpha$ of $\mathfrak{u}_{F}$. Hence

$$
\lim _{t \rightarrow \infty}\left[\pi\left(\exp \left(t\left(X+X^{\prime}\right)\right)\right) v_{H}\right]=\lim _{t \rightarrow \infty}\left[\pi\left(\exp \left(t\left(X+X^{\prime}\right)\right)\right) \widetilde{w}_{H}\right]=\left[v_{0}\right]
$$

and the second inclusion in (4.5) is established.

\subsection{Unimodularity issues under induction}

Let $P<G$ be a parabolic subgroup for which $P H$ is open.

Lemma 4.4. If $Z$ is unimodular then so is $P /(P \cap H)$.

Proof. As $P H$ is open, we can identify $P /(P \cap H)$ as an open subset of $Z$. The $G$-invariant measure on $Z$ then induces a $P$-invariant measure on $P /(P \cap H)$.

Next we observe the basic isomorphism

$$
Z_{P}=G_{P} / H_{P} \simeq P /(P \cap H) U_{P},
$$

which together with Lemma 4.4 allows us to compute the associated modular character $\Delta_{P}=\Delta_{Z_{P}}($ see $(3.26))$. 
LEMMA 4.5. Suppose that $Z$ is unimodular. Then

(1) The modular function for $Z_{P}=G_{P} / H_{P}$ is given by

$$
\Delta_{P}(h u)=\left|\operatorname{det} \operatorname{Ad}_{\mathfrak{u}_{P} / \mathfrak{u}_{P} \cap \mathfrak{h}}(h)\right|, \quad h \in P \cap H \text { and } u \in U_{P}
$$

(2) In particular, if $U_{P} \cap H=\{1\}$, then

$$
\Delta_{P}(h)=\left|\operatorname{det} \operatorname{Ad}_{\mathfrak{u}_{P}}(h)\right|, \quad h \in H_{P} .
$$

Proof. Since the modular function is trivial on the nilpotent group $U_{P}$, this follows from the exact sequence

$$
0 \longrightarrow \mathfrak{u}_{P} /\left(\mathfrak{u}_{P} \cap \mathfrak{h}\right) \longrightarrow \mathfrak{p} /(\mathfrak{p} \cap \mathfrak{h}) \longrightarrow \mathfrak{p} /\left(\mathfrak{p} \cap \mathfrak{h}+\mathfrak{u}_{P}\right) \longrightarrow 0
$$

of $(P \cap H)$-modules.

\section{Wave-front spaces and interlaced spherical subgroups}

The following notation will be used throughout. Recall the set of spherical roots

$$
S:=\left\{\sigma_{1}, \ldots, \sigma_{s}\right\} \subset \mathfrak{a}_{Z}^{*}
$$

such that

$$
\mathfrak{a}_{Z}^{-}=\left\{X \in \mathfrak{a}_{Z}: \sigma_{j}(X) \leqslant 0 \text { for } 1 \leqslant j \leqslant s\right\} .
$$

We let $\omega_{1}, \ldots, \omega_{s} \in \mathfrak{a}_{Z}$ be the dual basis to $S$, i.e. we require the following conditions:

- $\sigma_{i}\left(\omega_{j}\right)=\delta_{i j}, 1 \leqslant i, j \leqslant s$;

- $\omega_{i} \perp \mathfrak{a}_{Z, E}$.

This gives us the following coordinates of $\mathfrak{a}_{Z}$ :

$$
\begin{aligned}
\mathbb{R}^{s} \times \mathfrak{a}_{Z, E} & \longrightarrow \mathfrak{a}_{Z}, \\
(t, X) & \longmapsto \sum_{j=1}^{s} t_{j} \omega_{j}+X,
\end{aligned}
$$

with $\mathfrak{a}_{Z}^{-}$corresponding to pairs $(t, X)$ with $t_{j} \leqslant 0$. Moreover,

$$
\mathfrak{a}_{I}=\operatorname{span}\left\{\omega_{j}: j \notin I\right\}+\mathfrak{a}_{Z, E}, \quad I \subset S .
$$




\subsection{Wave-front spaces}

Let

$$
\mathfrak{a}^{-}:=\{X \in \mathfrak{a}: \alpha(X) \leqslant 0 \text { for all } \alpha \in \Pi\}
$$

be the closure of the negative Weyl chamber. Then the projection to $\mathfrak{a}_{Z}$ along $\mathfrak{a}_{H}$ maps $\mathfrak{a}^{-}$into $\mathfrak{a}_{Z}^{-}$. We recall that $Z$ is called wave-front provided the projection is onto, that is, provided $\mathfrak{a}_{Z}^{-}=\left(\mathfrak{a}^{-}+\mathfrak{a}_{H}\right) / \mathfrak{a}_{H}$.

Example 5.1. (a) All symmetric spaces are wave-front.

(b) The Gross-Prasad spaces (2.4) and (2.5) are wave-front.

(c) The triple space (see $\S 4.2 .2$ )

$$
G / H=(\mathrm{SL}(2, \mathbb{R}) \times \mathrm{SL}(2, \mathbb{R}) \times \mathrm{SL}(2, \mathbb{R})) / \mathrm{SL}(2, \mathbb{R})
$$

is wave-front.

(d) $\left(\mathfrak{s o}(3,4), G_{2}(\mathbb{R})\right)$ is wave-front.

(e) The series (2.1) and (2.2) are not wave-front.

A simple, but important feature of wave-front spaces is the fact which we record in the next lemma.

Lemma 5.2. If $G / H$ is wave-front then so is $G / H_{I} A_{I}$ for all $I \subset S$.

Proof. It follows from Proposition 3.2 (3) and (5) and from equation (5.2) that the wave-front-ness of $G / H_{I} A_{I}$ amounts to $\mathfrak{a}_{Z}^{-}+\mathfrak{a}_{I}+\mathfrak{a}_{H}=\mathfrak{a}^{-}+\mathfrak{a}_{I}+\mathfrak{a}_{H}$.

We continue by collecting a few but important facts of wave-front spaces. Recall from (3.8) that $S \subset \mathbb{Q} \geqslant 0[\Pi]$, and thus every $\sigma \in S$ has a unique presentation $\sigma=\sum_{\alpha \in \Pi} n_{\alpha} \alpha$ with $n_{\alpha} \in \mathbb{Q} \geqslant 0$. Accordingly, we define the support of $\sigma$ by

$$
\operatorname{supp}(\sigma):=\left\{\alpha \in \Pi: n_{\alpha}>0\right\} .
$$

We denote by $\left\{\omega_{\beta}^{\prime}\right\}_{\beta \in \Pi}$ the basis of $\mathfrak{a}$ dual to $\Pi$, i.e. $\alpha\left(\omega_{\beta}^{\prime}\right)=\delta_{\alpha, \beta}$. For every $\sigma \in S$ we let $\Pi_{\sigma} \subset \Pi$ be the set of $\alpha$ for which $\mathbb{R}^{+} \omega_{\alpha}^{\prime}+\mathfrak{a}_{H}=\mathbb{R}^{+} \omega_{\sigma}$. The following is then a reformulation of the definition of wave-front.

Lemma 5.3. For a real spherical space $Z$ the following conditions are equivalent:

(1) $Z$ is wave-front;

(2) $\Pi_{\sigma} \neq \varnothing$ for all $\sigma \in S$.

Furthermore, for each $\sigma \in S$,

$$
\Pi_{\sigma}=\operatorname{supp}(\sigma) \backslash \bigcup_{\sigma^{\prime} \neq \sigma} \operatorname{supp}\left(\sigma^{\prime}\right) .
$$


Proof. It follows from the assumption of wave-front that the extremal rays of the compression cone $\mathfrak{a}_{Z}^{-}$are generated by extremal rays of $\mathfrak{a}^{-}$. Hence $\Pi_{\sigma} \neq \varnothing$. Together with $\left(\mathfrak{a}^{-}+\mathfrak{a}_{H}\right) / \mathfrak{a}_{H} \subset \mathfrak{a}_{Z}^{-}$, we thus obtain the equivalence of (1) and (2). Let $\sigma \in S$. It follows from the definition that $\alpha \in \Pi_{\sigma}$ if and only if $\sigma\left(\omega_{\alpha}^{\prime}\right)>0$ and $\sigma^{\prime}\left(\omega_{\alpha}^{\prime}\right)=0$ for all $\sigma^{\prime} \neq \sigma$. Since $\alpha \in \operatorname{supp}\left(\sigma^{\prime}\right)$ if and only if $\sigma^{\prime}\left(\omega_{\alpha}^{\prime}\right)>0$, we obtain (5.3).

Lemma 5.4. Suppose that $Z$ is wave-front. Then $\Pi_{\sigma} \not \subset F_{Q}$ for all $\sigma \in S$.

Proof. (Cf. [34, Proof of Lemma 2.7.2].) We argue by contradiction and assume there exists $\sigma \in S$ with $\Pi_{\sigma} \subset F_{Q}$. Let $\alpha \in \Pi_{\sigma}$. Then $\alpha \in F_{Q}$, and hence $\alpha^{\vee} \in \mathfrak{a}_{H}$ by (4.1). Thus $\left\langle\alpha, \sigma^{\prime}\right\rangle=0$ for all $\sigma^{\prime} \in S$. Moreover, for $\sigma^{\prime} \neq \sigma$ and all $\beta \in \operatorname{supp}\left(\sigma^{\prime}\right)$ we have $\beta \neq \alpha$ by (5.3), and hence $\langle\alpha, \beta\rangle \leqslant 0$. Hence

$$
\alpha \perp \operatorname{supp}\left(\sigma^{\prime}\right), \quad \alpha \in \Pi_{\sigma} \text { and } \sigma^{\prime} \in S \backslash\{\sigma\} .
$$

Let $\sigma=\sum_{\alpha \in \Pi} n_{\alpha} \alpha=\sigma_{1}+\sigma_{2}$, where

$$
\sigma_{1}:=\sum_{\alpha \in \Pi_{\sigma}} n_{\alpha} \alpha \quad \text { and } \quad \sigma_{2}:=\sum_{\alpha \in \operatorname{supp}(\sigma) \backslash \Pi_{\sigma}} n_{\alpha} \alpha \text {. }
$$

With (5.3) and (5.4) we conclude now $\left\langle\alpha, \sigma_{2}\right\rangle=0$ for all $\alpha \in \Pi_{\sigma}$. As $\langle\alpha, \sigma\rangle=0$, we thus have $\left\langle\alpha, \sigma_{1}\right\rangle=0$ for all $\alpha \in \Pi_{\sigma}$. Hence $\left\langle\sigma_{1}, \sigma_{1}\right\rangle=0$, which contradicts $\Pi_{\sigma} \neq \varnothing$. This proves the lemma.

\subsection{Interlaced subgroups}

Let $P<G$ be a parabolic subgroup. We say that $H$ is interlaced by $P$ if

$$
U_{P} \subset H \subset P
$$

where $U_{P}$ is the unipotent radical of $P$. Note that, with $\bar{P}=\theta(P)$, it follows that $P H$ is open if $H$ is interlaced by $\bar{P}$.

Observe also that, if $H$ is interlaced by $P$, then $H_{P}=G_{P} \cap H$ and the Levi induced spherical space is $Z_{P}=G_{P} / H_{P} \simeq P / H$.

We now show that $H_{I}$ is non-trivially interlaced for all $I \nsubseteq S$ in case $Z$ is wave-front. With notation from above, we observe that we can use

$$
X_{I}=-\sum_{j \notin I} \omega_{j}
$$

for the element $X$ in (3.9). 
Assume that $Z$ is wave-front. To $I \subset S$ we now attach a minimal set $F=F_{I} \subset \Pi$ such that $F_{Q} \subset F$ and $H_{I}$ is interlaced by $\bar{P}_{F}$. We define $F_{I}$ to be the complement of $J_{I} \subset \Pi$, where

$$
J_{I}:=\bigcup_{\sigma \notin I}\left(\Pi_{\sigma} \backslash F_{Q}\right) .
$$

Proposition 5.5. Assume that $Z$ is wave-front. Let $I \subset S$ and $F=F_{I}$ be as defined above. Then there exists an element $Y_{I} \in \mathfrak{a}_{F}$ such that $Y_{I}+\mathfrak{a}_{H}=X_{I}$ and $\alpha\left(Y_{I}\right)<0$ for all $\alpha \in \Sigma_{\mathfrak{u}} \backslash\langle F\rangle$. In particular,

$$
\overline{\mathfrak{p}}_{F}=\mathfrak{m}+\mathfrak{a}+\bigoplus_{\substack{\alpha \in \Sigma \\ \alpha\left(Y_{I}\right) \leqslant 0}} \mathfrak{g}^{\alpha}
$$

and $G_{F}=Z_{G}\left(Y_{I}\right)$.

Proof. It follows from Lemma 5.4 and the definition of $\Pi_{\sigma}$ that the coset

$$
\sum_{\alpha \in \Pi_{\sigma} \backslash F_{Q}} \omega_{\alpha}^{\prime}+\mathfrak{a}_{H}
$$

is a positive multiple of $\omega_{\sigma}$ for each $\sigma \notin I$. Thus, for suitable constants $c_{\alpha}>0$, the element $Y_{I}=-\sum_{\alpha \in J_{I}} c_{\alpha} \omega_{\alpha}^{\prime}$ has the desired property.

Corollary 5.6. Let $F=F_{I} \subset \Pi$ be as above. Then $\langle I\rangle=\mathbb{Q} \geqslant 0[F] \cap\langle S\rangle, \mathfrak{a}_{I}=\mathfrak{a}_{F}+\mathfrak{a}_{H}$ and

$$
\left(G_{F} \cap H\right)_{e} \bar{U}_{F} \subset H_{I} \subset \bar{P}_{F} .
$$

In particular, $H_{I}$ is interlaced by $\bar{P}_{F}$.

Proof. The first two statements follow immediately from Proposition 5.5. It also follows that

$$
\alpha+\beta \in\langle I\rangle \quad \text { if and only if } \alpha, \beta \in\langle F\rangle
$$

for all $\alpha \in \Sigma_{\mathfrak{u}}$ and $\beta \in \Sigma_{\mathfrak{u}} \cup\{0\}$ with $\alpha+\beta \in \mathbb{N}_{0}[S]$.

As $F_{Q} \subset F$, we have $\mathfrak{l} \mathfrak{h} \subset \mathfrak{g}_{F}$. It then follows from (5.7) and the descriptions of $\mathfrak{h}$ and $\mathfrak{h}_{I}$ by means of the maps $T$ and $T_{I}$ that

$$
\mathfrak{g}_{F} \cap \mathfrak{h}+\overline{\mathfrak{u}}_{F} \subset \mathfrak{h}_{I} \subset \overline{\mathfrak{p}}_{F}
$$

This implies (5.6).

Remark 5.7. The property of being interlaced may also hold in cases where $Z$ is not wave-front. For example, the spherical subgroup $\bar{N}$ is interlaced by $P_{\min }$, but $Z=G / \bar{N}$ is not wave-front. 


\section{Power series expansions of generalized matrix coefficients on the compression cone}

Given a Harish-Chandra module $V$ for the pair $(\mathfrak{g}, K)$, we write $V^{\infty}$ for its unique smooth moderate growth Fréchet completion, and $V^{-\infty}$ for the strong dual of $V^{\infty}$. Both $V^{\infty}$ and $V^{-\infty}$ are $G$-modules. With a pair $(v, \eta) \in V^{\infty} \times V^{-\infty}$ we associate the generalized matrix coefficient

$$
m_{v, \eta}(g):=\eta\left(g^{-1} \cdot v\right), \quad g \in G
$$

which is a smooth function on $G$.

Let $Z=G / H$ be a real spherical space. We say that $V$ is $H$-spherical provided that $\left(V^{-\infty}\right)^{H} \neq\{0\}$. We recall (see [27, Theorem 3.2]) that

$$
\operatorname{dim}\left(V^{-\infty}\right)^{H} \leqslant \operatorname{dim}(V /(\mathfrak{l} \cap \mathfrak{h}+\overline{\mathfrak{u}}) V)<\infty .
$$

The group $A_{Z, E}$ naturally acts on the vector space $\left(V^{-\infty}\right)^{H}$. Note, however, that the action need not be semi-simple. In the sequel we only consider $\eta \in\left(V^{-\infty}\right)^{H}$, which are eigenvectors of $A_{Z, E}$.

In this case, we call $(V, \eta)$ an $H$-spherical pair. We then regard $m_{v, \eta}$ as an element of $C^{\infty}(G / H)$.

Remark 6.1. For our objective, namely to understand tempered representations, the assumption that $\eta$ is an $A_{Z, E}$-eigenvector is no loss of generality. In order to justify that we recall the abstract Plancherel theorem for the left regular action $L$ of $G$ on $L^{2}(Z)$ :

$$
L^{2}(Z) \simeq \int_{\hat{G}}^{\oplus} \mathcal{M}_{\pi} \otimes \mathcal{H}_{\pi} d \mu(\pi)
$$

with $\mathcal{M}_{\pi} \subset\left(\mathcal{H}_{\pi}^{-\infty}\right)^{H}$, the multiplicity space equipped with some inner product (see [21, $\S 5.1]$ for the notation). Our interest lies in those linear forms $\eta$ which belong to $\mathcal{M}_{\pi}$ and the corresponding matrix coefficients $m_{v, \eta} \in L^{2}(Z)$ obtained from (6.1) with $v \in \mathcal{H}_{\pi}^{\infty}$.

If $\mu_{Z}$ denotes the Haar-measure on $Z$, then there exists a positive character $\psi$ of $A_{Z, E}$, the restriction of the modular function $\hat{\Delta}$ of $(3.26)$ for $\widehat{Z}=G / H A_{Z, E}$, such that $\mu_{Z}(E a)=\psi(a) \mu_{Z}(E)$ for all measurable sets $E \subset Z$ and $a \in A_{Z, E}$. Hence, we obtain a unitary representation $R$ of $A_{Z, E}$ by

$$
(R(a) f)(z)=\sqrt{\psi(a)} f(z a), \quad f \in L^{2}(Z), z \in Z \text { and } a \in A_{Z, E}
$$

As the representations $R$ and $L$ commute, we obtain from (6.1) for $\mu$-almost all $\pi$ that the natural action of $A_{Z, E}$ on $\left(V^{-\infty}\right)^{H}$ restricts to a unitary action on $\mathcal{M}_{\pi}$. In particular, 
this action on $\mathcal{M}_{\pi}$ is semi-simple. Moreover, if $\eta \in \mathcal{M}_{\pi}$ is an eigenvector with eigenvalue $a^{\chi}$, then $f=m_{v, \eta}$ is an eigenvector for $R(a)$ with eigenvalue $\psi(a)^{1 / 2} a^{\chi}$. Hence,

$$
e^{\operatorname{Re} \chi}=\hat{\Delta}(a)^{-1 / 2}
$$

for all $\chi \in \mathfrak{a}_{Z, E, \mathbb{C}}^{*}$ appearing in the spectrum of the $\mathfrak{a}_{Z, E}$-module $\mathcal{M}_{\pi}$.

Recall the set $\mathcal{W}=\mathcal{F F}^{\prime}$, where $\mathcal{F}^{\prime} \subset \widehat{H}_{0}$ is a set of representatives of the component group $\widehat{H}_{0} / H A_{Z, E}$. For $w \in \mathcal{W}$ we set $\eta_{w}:=w \cdot \eta=\eta\left(w^{-1} \cdot\right)$, and observe that $\eta_{w}$ is $H_{w}$-fixed. We thus have an isomorphism

$$
\begin{aligned}
&\left(V^{-\infty}\right)^{H} \longrightarrow\left(V^{-\infty}\right)^{H_{w}}, \\
& \eta \longmapsto \eta_{w} .
\end{aligned}
$$

We recall that $L \cap H<H_{w}$ for all $w \in \mathcal{W}$. Observe the obvious identity

$$
m_{v, \eta}\left(g w \cdot z_{0}\right)=m_{v, \eta_{w}}\left(g \cdot z_{w}\right), \quad g \in G,
$$

where $z_{w}$ denotes the origin of the homogeneous space $Z_{w}=G / H_{w}$.

Lemma 6.2. Let $\eta \in\left(V^{-\infty}\right)^{H}$ be an $A_{Z, E}$-eigenvector corresponding to a character $\chi \in \mathfrak{a}_{Z, E, \mathbb{C}}^{*}$, i.e. $a \cdot \eta=a^{\chi} \eta$ for $a \in A_{Z, E}$. Then, for all $w \in \mathcal{W}$,

$$
a \cdot \eta_{w}=a^{\chi} \eta_{w}, \quad a \in A_{Z, E} .
$$

Proof. Recall from Lemma 3.5 that $a w=w a h$ for some $h \in H$. From this, the assertion follows.

From this lemma, we obtain the identity

$$
m_{v, \eta}\left(a b w \cdot z_{0}\right)=a^{\chi} m_{v, \eta}\left(b w \cdot z_{0}\right)
$$

for all $a \in A_{Z, E}, b \in A_{Z}$ and $w \in \mathcal{W}$.

In the sequel, we realize $\mathfrak{a}_{Z}$ inside of $\mathfrak{a}$ as $\mathfrak{a}_{H}^{\perp}$. Let $a_{0} \in A$ be such that $a_{0} A_{H}$ lies in the interior of $A_{Z}^{-}$. It follows from [27, Lemma 5.1] that we can choose this element $a_{0}$ such that, in addition, there is a relatively compact open neighbourhood $U_{A}$ of $\mathbf{1}$ in $A_{Z}$, with

$$
\mathfrak{k}+\operatorname{Ad}(a) \mathfrak{h}_{w}+\mathfrak{a}_{Z}=\mathfrak{g}
$$

for all $a \in a_{0} U_{A} \cdot A_{Z}^{-}$and $w \in \mathcal{W}$. What we have fixed so far are the tori $A$ and $A_{Z}$. These are invariant under conjugation by $a_{0}$, and hence we are free to replace $K$ by $\operatorname{Ad}\left(a_{0}^{-1}\right) K$. We may thus assume that (6.5) holds for all $a \in U_{A} \cdot A_{Z}^{-}$. We note that such a change 
leaves the neighborhood $U_{A}$ unchanged. Moreover, we still have the freedom of making further replacements of this type, but now only by elements from $A_{Z}^{-}$.

What we actually use is the conjugated form

$$
\operatorname{Ad}(a)^{-1} \mathfrak{k}+\mathfrak{a}_{Z}+\mathfrak{h}_{w}=\mathfrak{g},
$$

and we express elements from the Lie algebra (and via Poincaré-Birkhoff-Witt also elements from $\mathcal{U}(\mathfrak{g}))$ via this decomposition. We then let $\mathcal{U}(\mathfrak{g})$ act on smooth functions on $G$ via right differentiation. Functions on $Z_{w}=G / H_{w}$ will be considered as functions on $G$ which are right $H_{w}$-invariant. The functions of our concern are the smooth matrix coefficients $g \mapsto m_{v, \eta_{w}}(g)$ when restricted to functions on $U_{A} \cdot A_{Z}^{-}$.

\subsection{Holomorphic decompositions of the universal enveloping algebra}

The fact that matrix coefficients $m_{v, \eta}$ admit power series expansions when restricted to $A^{-} A_{H} / A_{H} \subset A_{Z}^{-}$rests on several decomposition theorems for the universal algebra $\mathcal{U}(\mathfrak{g})$ of $\mathfrak{g}$. These results were in provided in $[27, \S 5]$, and are parallel to the special case where $Z$ is a group (see [16, Chapter VIII, $\S 7$ and $\S 8]$ ).

We develop here the framework for a theory, which extends the results from [27] by allowing the expansions to take place on the full compression cone $A_{Z}^{-}$.

Recall the coordinates (5.1) of $\mathfrak{a}_{Z}$ and fix a basis $\nu_{1}, \ldots, \nu_{e}$ of $\mathfrak{a}_{Z, E}^{*}$. We write $D$ for the open unit disc in $\mathbb{C}$ and write $D^{\times}=D \backslash\{0\}$. Let $\mathcal{D}=D^{s} \times\left(\mathbb{C}^{*}\right)^{e}$ and $\mathcal{D}^{\times}=\left(D^{\times}\right)^{r} \times\left(\mathbb{C}^{*}\right)^{e}$. For $r>1$ we realize $A_{Z}^{-}$in $r \mathcal{D}^{\times}$via the map

$$
\begin{aligned}
A_{Z}^{-} & \longrightarrow r \mathcal{D}^{\times}, \\
& a \longmapsto\left(a^{\sigma_{1}}, \ldots, a^{\sigma_{s}}, a^{\nu_{1}}, \ldots, a^{\nu_{e}}\right) .
\end{aligned}
$$

It is no loss of generality to assume that $U_{A}$ and $r$ are chosen such that

$$
r \mathcal{D} \cap\left(\mathbb{R}_{>0}\right)^{n}=U_{A} \cdot A_{Z}^{-} .
$$

We write $\mathcal{O}(\mathcal{D})$ for the ring of holomorphic functions on $\mathcal{D}$ and $\mathcal{O}_{1}(\mathcal{D})$ for the subring of functions which are independent of the variable from $\left(\mathbb{C}^{*}\right)^{e}$.

Let $\left(W_{l}\right)_{l}$ be a basis for $\mathfrak{h}_{\text {lim }}=\mathfrak{l} \cap \mathfrak{h}+\overline{\mathfrak{u}}$ such that either $W_{l} \in \mathfrak{l} \cap \mathfrak{h}$ or $W_{l}=X_{-\alpha} \in \mathfrak{g}^{-\alpha}$ for some $\alpha \in \Sigma_{\mathfrak{u}}$. Further, let $\left(V_{k}\right)_{k}$ be a basis for $\mathfrak{a}_{Z}$ and $\left(U_{j}\right)_{j}$ be a linear independent set in $\mathfrak{k}$ which complements $\left(W_{l}, V_{k}\right)_{l, k}$ to a basis for $\mathfrak{g}$.

For $a \in A_{Z}$ we set $W_{l}(a):=W_{l}$ if $W_{l} \in \mathfrak{h} \cap \mathfrak{l}$, and, with the notation of (3.3),

$$
W_{l}(a):=X_{-\alpha}+\sum_{\beta} a^{\alpha+\beta} X_{\alpha, \beta}=a^{\alpha} \operatorname{Ad}(a)\left(X_{-\alpha}+T\left(X_{-\alpha}\right)\right)
$$


if $W_{l}=X_{-\alpha}$. Then $\left(W_{l}(a)\right)_{l}$ is a basis of $\operatorname{Ad}(a) \mathfrak{h}$ for all $a \in A_{Z}$, and the transition between $\left(W_{l}(a b)\right)_{l}$ and $\left(\operatorname{Ad}(b) W_{l}(a)\right)_{l}$ is by a diagonal matrix depending only on $b \in A_{Z}$. In particular, $W_{l}(a)=W_{l}(a b)$ for $b \in A_{Z, E}$.

Now note the following, as a consequence of our normalization (3.7) of $S$ : The set of vectors $\left(W_{l}(a), V_{k}, U_{j}\right)_{l, k, j}$ is expressable in terms of the basis $\left(W_{l}, V_{k}, U_{j}\right)_{l, k, j}$ through a transition matrix of the form $\mathbf{1}+P(a)$, where each entry of $P(a)$ extends to a polynomial in $\mathcal{O}_{1}(r \mathcal{D})$ without constant term. It follows that there exists $a_{0} \in A_{Z}^{-}$such that this matrix is invertible for all $a \in a_{0} U_{A} A_{Z}^{-}$, and such that the entries of the inverse matrix again belong to $\mathcal{O}_{1}(r \mathcal{D})$ as functions of $a_{0}^{-1} a$.

Lemma 6.3. There exists $a_{0} \in A_{Z}^{-}$with the following property. For every $X \in \mathfrak{g}$ there exist functions $f_{j}, g_{k}, h_{l} \in \mathcal{O}_{1}(r \mathcal{D})$ such that

$$
X=\sum_{j} f_{j}(a) U_{j}+\sum_{k} g_{k}(a) V_{k}+\sum_{l} h_{l}(a) W_{l}\left(a_{0} a\right)
$$

for all $a \in U_{A} \cdot A_{Z}^{-}$. Moreover, the values at zero of these functions are given by

$$
X=\sum_{j} f_{j}(0) U_{j}+\sum_{k} g_{k}(0) V_{k}+\sum_{l} h_{l}(0) W_{l} .
$$

In particular, $g_{k}(0)=0$ for all $k$ if $X \in \mathfrak{u}$.

Proof. This is clear by the preceeding remarks. The statement for $X \in \mathfrak{u}$ follows from the fact that $\mathfrak{u} \subset \mathfrak{k}+\mathfrak{h}_{\text {lim }}=\operatorname{span}\left\{U_{j}, W_{l}\right\}$.

Using induction on the degree, an immediate consequence of the preceding lemma is the following.

Corollary 6.4. (See [27, Lemma 5.3]) For every $u \in \mathcal{U}(\mathfrak{g})$ there exist $\mathbf{U}_{j} \in \mathcal{U}(\mathfrak{k})$, $\mathbf{V}_{j} \in \mathcal{U}\left(\mathfrak{a}_{Z}\right), \mathbf{W}_{j}(a) \in \mathcal{U}(\operatorname{Ad}(a) \mathfrak{h})$ and $f_{j} \in \mathcal{O}_{1}(r \mathcal{D})$ such that

$$
u=\sum_{j=1}^{p} f_{j}\left(a_{0}^{-1} a\right) \mathbf{U}_{j} \mathbf{V}_{j} \mathbf{W}_{j}(a), \quad a \in a_{0} U_{A} \cdot A_{Z}^{-} .
$$

Moreover, each $\mathbf{W}_{j}$ is a product of $W_{i}$ 's, as a function of a, and

$$
\operatorname{deg}\left(\mathbf{U}_{j}\right)+\operatorname{deg}\left(\mathbf{V}_{j}\right)+\operatorname{deg}\left(\mathbf{W}_{j}(a)\right) \leqslant \operatorname{deg}(u) .
$$

For every $n \in \mathbb{N}_{0}$ we denote by $\mathcal{U}(\mathfrak{g})_{n}$ the subspace of $\mathcal{U}(\mathfrak{g})$ consisting of elements of degree at most $n$.

We use $\mathcal{Z}(\mathfrak{g})$, the center of $\mathcal{U}(\mathfrak{g})$, to replace the $\mathbf{V}_{j}$ 's in the decomposition (6.7). The formal statement is as follows. 
LEMma 6.5. There exists a finite-dimensional subspace $\mathcal{Y} \subset \mathcal{U}\left(\mathfrak{a}_{Z}\right)$ with the following property: For all $n \in \mathbb{N}_{0}$ there exists an element $a_{n} \in A_{Z}^{-}$such that for every $u \in \mathcal{U}(\mathfrak{g})_{n}$ there exist $\mathbf{U}_{j} \in \mathcal{U}(\mathfrak{k}), \mathbf{Y}_{j} \in \mathcal{Y}, z_{j} \in \mathcal{Z}(\mathfrak{g}), \mathbf{W}_{j}(a) \in \mathcal{U}(\operatorname{Ad}(a) \mathfrak{h})$ and $f_{j} \in \mathcal{O}_{1}(r \mathcal{D})$ such that

$$
u=\sum_{j=1}^{p} f_{j}\left(a_{n}^{-1} a\right) \mathbf{U}_{j} \mathbf{Y}_{j} \mathbf{W}_{j}(a) z_{j}, \quad a \in a_{n} U_{A} \cdot A_{Z}^{-} .
$$

Moreover, each $\mathbf{W}_{j}$ is a product of $W_{i}$ 's, as a function of a.

Proof. The proof, which goes by induction on the index $n$, is analogous to that of [27, Lemma 5.5]. As explained there, we can reduce to the case where $u=X v$, with $X \in \mathfrak{u}$ and $v \in \mathcal{U}(\mathfrak{g})_{n-1}$ (note that, by [27, equation (5.9)], we have $T_{m^{\prime \prime}} \in \mathfrak{h} \cap \mathfrak{l}$ for the element denoted like that, and hence this element is a fixed linear combination of the $W_{l}(a)$ 's for all $a$ ). In fact, it is more convenient to consider $u=v X$, which is equivalent as the induction hypothesis applies to $[X, v]$. We use Lemma 6.3 and obtain, for $a \in a_{0} U_{A} \cdot A_{Z}^{-}$,

$$
u=\sum_{j} f_{j}\left(a_{0}^{-1} a\right) v U_{j}+\sum_{k} g_{k}\left(a_{0}^{-1} a\right) v V_{k}+\sum_{l} h_{l}\left(a_{0}^{-1} a\right) v W_{l}(a) .
$$

The terms with $W_{l}$ obtain the desired form (for any $a_{n} \in a_{n-1} A_{Z}^{-}$) immediately from the induction hypothesis applied to $v$. By replacing $v U_{j}$ with $U_{j} v$, which we are allowed to do by the induction hypothesis, we can say the same for the terms with $U_{j}$. Finally, since $X \in \mathfrak{u}$, we have from Lemma 6.3 that $g_{k}(0)=0$ for all $k$. This allows us to use the argument in the final lines of the proof of [27, Lemma 5.5], and obtain (6.8) with an appropriate $a_{n}$.

The main conclusion then is the following.

Proposition 6.6. Let $u \in \mathcal{U}\left(\mathfrak{a}_{Z}\right)_{n}$ and let $a_{n} \in A_{Z}^{-}$be as in the preceding lemma. Then, there exist $\mathbf{U}_{j} \in \mathcal{U}\left(\operatorname{Ad}\left(a_{n}\right)^{-1} \mathfrak{k}\right), \mathbf{Y}_{j} \in \mathcal{Y}, z_{j} \in \mathcal{Z}(\mathfrak{g})$ and $f_{j} \in \mathcal{O}_{1}(r \mathcal{D})$ such that

$$
u=\sum_{j=1}^{p} f_{j}(a)\left(\operatorname{Ad}(a)^{-1} \mathbf{U}_{j}\right) \mathbf{Y}_{j} z_{j} \bmod \mathcal{U}(\mathfrak{g}) \mathfrak{h}, \quad a \in U_{A} \cdot A_{Z}^{-}
$$

Proof. Let $a \in U_{A} \cdot A_{Z}^{-}$. Then

$$
u=\sum_{j=1}^{p} f_{j}(a) \mathbf{U}_{j} \mathbf{Y}_{j} \mathbf{W}_{j}\left(a_{n} a\right) z_{j}
$$

by (6.8). Apply $\operatorname{Ad}\left(a_{n} a\right)^{-1}$ and observe that $\operatorname{Ad}\left(a_{n} a\right)^{-1} u=u$.

Remark 6.7. It is clear that Proposition 6.6 holds as well if $\mathfrak{h}$ is replaced by $\mathfrak{h}_{w}$ for $w \in \mathcal{W}$ 
By a hypersurface in $A_{Z}$ we understand a level set of a non-zero real analytic function on $A_{Z}$. Notice that a hypersurface is a closed subset of measure zero.

Then there is the following counterpart of Proposition 6.6 which does not depend on the filtration degree $n$ but carries no information on the coefficients.

Lemma 6.8. There exists a countable union of hypersurfaces $\mathcal{S} \subset A_{Z}$ such that

$$
\mathcal{U}(\mathfrak{g})=\mathcal{U}\left(\operatorname{Ad}(a)^{-1} \mathfrak{k}\right) \mathcal{Y} \mathcal{Z}(\mathfrak{g}) \mathcal{U}(\mathfrak{h}), \quad a \in A_{Z} \backslash \mathcal{S} .
$$

Proof. The proof of $[27$, Lemma 5.5] shows that for every $n \in \mathbb{N}$ there exists a finite union of hypersurfaces $\mathcal{S}_{n} \subset A_{Z}$ such that

$$
\mathcal{U}(\mathfrak{g})_{n} \subset \mathcal{U}\left(\operatorname{Ad}(a)^{-1} \mathfrak{k}\right) \mathcal{Y} \mathcal{Z}(\mathfrak{g}) \mathcal{U}(\mathfrak{h}) \quad a \in A_{Z} \backslash \mathcal{S}_{n} .
$$

We let now $n_{0}:=1+\max \{\operatorname{deg} Y: Y \in \mathcal{Y}\}$. As described in (6.5), we are free to replace $K$ by $\operatorname{Ad}\left(a_{0}^{-1}\right) K$ without disturbing other choices. We do this with the above element $a_{n_{0}}$. Then we obtain that (6.9) holds for all $u \in \mathcal{U}(\mathfrak{a})_{n_{0}}$ with $\mathbf{U}_{j} \in \mathcal{U}(\mathfrak{k})$. Further, Lemma 6.8 allows us to request in addition that

$$
\mathcal{U}(\mathfrak{g})=\mathcal{U}(\mathfrak{k}) \mathcal{Y} \mathcal{Z}(\mathfrak{g}) \mathcal{U}\left(\left(\mathfrak{h}_{w}\right)_{I}\right)
$$

for all $I \subset S$ and $w \in \mathcal{W}$.

We recapitulate that we have shown that there exists a Cartan involution for which (6.5) holds for all $a \in U_{A} \cdot A_{Z}^{-}$, for which (6.9) is valid for $u \in \mathcal{U}(\mathfrak{a})_{n_{0}}$ with $\mathbf{U}_{j} \in \mathcal{U}(\mathfrak{k})$, and for which (6.11) holds for all $I \subset S$ and $w \in \mathcal{W}$. We shall refer to the corresponding maximal compact subgroup $K$ as regular.

We recall that, in case $\mathfrak{h}=\mathfrak{n}$, the decomposition (6.11) reduces to the CasselmanOsborne lemma ([36, Proposition 3.7.1]), and that it implies the following finiteness result (see [1, Proposition 4.2]).

Lemma 6.9. With a regular choice of $K$, every Harish-Chandra module $V$ is finitely generated as $\mathcal{U}\left(\left(\mathfrak{h}_{w}\right)_{I}\right)$-module, for all $w \in \mathcal{W}$ and $I \subset S$. In particular, $H_{0}\left(V,\left(\mathfrak{h}_{w}\right)_{I}\right)=$ $V /\left(\mathfrak{h}_{w}\right)_{I} V$ is finite-dimensional.

We now fix once and for all a Cartan involution for which $K$ is regular.

\subsection{Power series expansions for $K$-finite vectors}

Following [10] and [2], we developed in [27, §5] a theory of power series expansions for $K$ finite matrix coefficients $m_{v, \eta_{w}}$, which we briefly summarize. Attached to an $H$-spherical pair $(V, \eta)$, there exists a number $d \in \mathbb{N}$ and for each $v \in V$ a finite set

$$
\mathcal{E}=\left\{\Lambda_{1}, \ldots, \Lambda_{l}\right\} \subset \mathfrak{a}_{Z, \mathbb{C}}^{*}
$$


such that for every $w \in \mathcal{W}$ one has an expansion

$$
m_{v, \eta}\left(a w \cdot z_{0}\right)=\sum_{j=1}^{l} \sum_{\alpha \in \mathbb{N}_{0}[S]} a^{\Lambda_{j}+\alpha} q_{\alpha, j, w}(\log a)
$$

with absolute convergence for all $a \in U_{A} A_{Z}^{-}$. The expansion (6.12) is derived in analogy to [27, Corollary 5.7] when we use Proposition 6.6 (with the regular choice of $\mathfrak{k}$ ), and Remark 6.7 instead of [27, Lemma 5.5]. Here we also used that $\eta$ is an $A_{Z, E}$-eigenvector to separate off the $A_{Z, E}$-behavior of $m_{v, \eta_{w}}$ in advance (see (6.4)), i.e.

$$
\left.\Lambda_{j}\right|_{\mathfrak{a}_{Z, E}}=\chi, \quad 1 \leqslant j \leqslant l .
$$

We can arrange that no mutual difference between two elements from $\mathcal{E}$ belongs to $\mathbb{Z}[S]$. The individual terms in (6.12) are then unique.

The set of exponents of $v$ along $A_{Z}^{-}$is then the set

$$
\Xi_{v}=\left\{\xi \in \mathfrak{a}_{Z, \mathbb{C}}^{*}: \xi=\Lambda_{j}+\alpha \text { for some } \alpha, j \text { and } w \text { with } q_{\alpha, j, w} \neq 0\right\}
$$

and the set of leading exponents is

$$
\mathcal{E}_{\text {lead }, v}=\left\{\xi \in \Xi_{v}: \xi-\alpha \notin \Xi_{v} \text { for all } \alpha \in \mathbb{N}_{0}[S] \backslash\{0\}\right\} \text {. }
$$

Let $\mathfrak{a} \oplus \mathfrak{b} \subset \mathfrak{g}$ be a full Cartan subalgebra, and let $W_{\mathbb{C}}$ denote the corresponding Weyl group for the root system of $(\mathfrak{a} \oplus \mathfrak{b})_{\mathbb{C}}$ in $\mathfrak{g}_{\mathbb{C}}$. As in [16, Theorem 8.33] (see also [2, Theorem 2.4 and Proposition 4.1]) we find, in case $V$ admits an infinitesimal character, that

$$
\mathcal{E}_{\text {lead }, v} \subset\left\{\left.u \Lambda\right|_{\mathfrak{a}_{Z}}: u \in W_{\mathbb{C}}\right\}+\varrho_{P},
$$

where $\Lambda \in(\mathfrak{a} \oplus \mathfrak{b})_{\mathbb{C}}^{*}$ is the infinitesimal character of $V$. As every Harish-Chandra module has a finite composition series, we conclude that $\mathcal{E}_{\text {lead }, v}$ is finite.

Notice that the set on the right side of the inclusion (6.14) is independent of $v$. We define

$$
\Xi:=\bigcup_{v} \Xi_{v}
$$

and

$$
\mathcal{E}_{\text {lead }}:=\left\{\xi \in \Xi: \xi-\alpha \notin \Xi \text { for all } \alpha \in \mathbb{N}_{0}[S] \backslash\{0\}\right\}
$$

It follows that

$$
\mathcal{E}_{\text {lead }} \subset\left\{\left.u \Lambda\right|_{\mathfrak{a}_{Z}}: u \in W_{\mathbb{C}}\right\}+\varrho_{P},
$$


and in particular it is a finite set. Now

$$
\Xi \subset \mathcal{E}_{\text {lead }}+\mathbb{N}_{0}[S]
$$

and the expansion (6.12) reads

$$
m_{v, \eta}\left(a w \cdot z_{0}\right)=\sum_{\xi \in \Xi} a^{\xi} q_{\eta, v, \xi, w}(\log a),
$$

with polynomials $q_{\eta, v, \xi, w}$ of degree $\leqslant d$ which depend linearly on $\eta$ and $v$. To simplify notation, we shall write $q_{\xi, w}$ instead of $q_{\eta, v, \xi, w}$, whenever it is clear from the context that $\eta$ and $v$ have been fixed. From (6.3) we see that

$$
q_{\eta_{w}, v, \xi, 1}=q_{\eta, v, \xi, w}
$$

for all $w \in \mathcal{W}$. Note that all $\sigma \in S$ vanish on $\mathfrak{a}_{Z, E}$, and hence, by (6.13),

$$
\left.\xi\right|_{\mathfrak{a}_{Z, E}}=\chi, \quad \xi \in \Xi
$$

To the pair $(V, \eta)$ we attach now an element $\Lambda_{V, \eta} \in \mathfrak{a}_{Z}^{*}$ as follows. Recalling (5.1), we let

$$
\Lambda_{V, \eta}\left(\omega_{j}\right):=\min _{\xi \in \Xi} \operatorname{Re} \xi\left(\omega_{j}\right)=\min _{\lambda \in \mathcal{E}_{\text {lead }}} \operatorname{Re} \lambda\left(\omega_{j}\right)
$$

and define

$$
\left.\Lambda_{V, \eta}\right|_{\mathfrak{a}_{Z, E}}=\operatorname{Re} \chi .
$$

Remark 6.10. The exponent $\Lambda_{V}$ defined in [24, Theorem 5.8] does not depend on the particular $\eta$. The exponent $\Lambda_{V, \eta}$ defined here is an invariant of the pair $(V, \eta)$.

\subsection{Upper and lower bounds on matrix coefficients}

We regard $\Lambda_{V, \eta}$ as an element in $\mathfrak{a}^{*}$ trivial on $\mathfrak{a}_{H}$. Hence (6.17) yields, after possibly shrinking $U_{A}$, for all $v \in V$ a constant $C_{v}>0$ such that

$$
\left|m_{v, \eta}\left(a w \cdot z_{0}\right)\right| \leqslant C_{v}(1+\|\log a\|)^{d} a^{\Lambda_{V, \eta}}, \quad a \in U_{A} \cdot A_{Z}^{-},
$$

for all $w \in \mathcal{W}$.

A sharper upper bound is possible along rays in $A_{Z}^{-}$. By (5.1) and (6.19)-(6.21), we find for $a \in A_{Z}^{-}$that

$$
\mathbf{w}_{\eta}(a):=\max _{\xi \in \Xi} a^{\operatorname{Re} \xi} \leqslant a^{\Lambda_{V, \eta}}, \quad a \in A_{Z}^{-} .
$$


Let $\mathbf{S}\left(\mathfrak{a}_{Z}^{-}\right)$be the intersection of the unit sphere with $\mathfrak{a}_{Z}^{-}$, then

$$
\mathbf{w}_{\eta}(\exp (t X))=e^{t \lambda_{X}}, \quad t \geqslant 0
$$

where

$$
\lambda_{X}:=\max _{\xi \in \Xi} \operatorname{Re} \xi(X), \quad X \in \mathbf{S}\left(\mathfrak{a}_{Z}^{-}\right) .
$$

Observe also that $\mathbf{w}_{\eta}$ is continuous and a weight on $A_{Z}^{-}$, that is, for all compact subsets $C_{A} \subset A_{Z}^{-}$there exists a constant $c>0$ such that

$$
\frac{1}{c} \mathbf{w}_{\eta}(a) \leqslant \mathbf{w}_{\eta}(b a) \leqslant c \mathbf{w}_{\eta}(a), \quad a \in A_{Z}^{-} \text {and } b \in C_{A} .
$$

Furthermore, let

$$
\Xi_{X}=\left\{\xi \in \Xi: \operatorname{Re} \xi(X)=\lambda_{X}\right\}
$$

and define

$$
d_{X}=\max _{\substack{v \in V \\ \xi \in \Xi_{X} \\ w \in \mathcal{W}}} \operatorname{deg}\left(t \mapsto q_{v, \xi, w}(t X)\right)
$$

and

$$
\mathbf{d}_{\eta}(\exp (t X)):=t^{d_{X}}, \quad t \geqslant 0
$$

Lemma 6.11. Let $X \in \mathbf{S}\left(\mathfrak{a}_{Z}^{-}\right)$. For each $v \in V$ there exists a constant $C=C_{v, X}>0$ such that

$$
\left|m_{v, \eta}\left(\exp (t X) w \cdot z_{0}\right)\right| \leqslant C \mathbf{w}_{\eta}(\exp (t X)) \mathbf{d}_{\eta}(\exp (t X))
$$

for all $t \geqslant 1$ and $w \in \mathcal{W}$.

Proof. With (6.17) we write

$$
m_{v, \eta}\left(a_{t} w \cdot z_{0}\right)=\sum_{\xi \in \Xi_{X}} a_{t}^{\xi} q_{v, \xi, w}(t X)+\sum_{\xi \in \Xi \backslash \Xi_{X}} a_{t}^{\xi} q_{v, \xi, w}(t X)
$$

where $a_{t}=\exp (t X)$. The polynomials $q_{v, \xi, w}$ in the first sum have degree at most $d_{X}$, and hence the asserted bound follows for this part. In the second sum the polynomials may have higher degrees (up to $d$ ), but as the exponentials are bounded by $e^{\lambda_{X}^{\prime}}$ for some $\lambda_{X}^{\prime}<\lambda_{X}$, this term is in fact of the form $o\left(e^{t \lambda_{X}}\right)$ for $t \rightarrow \infty$.

The bound in (6.24) is essentially optimal. The formal statement will be given in Proposition 6.13. However, we first need a lemma. 
Lemma 6.12. Let $X \in \mathbf{S}\left(\mathfrak{a}_{Z}^{-}\right)$and let $\lambda_{X}$ and $d_{X}$ be defined as above. Then the set

$$
\Lambda=\left\{\xi(X): \xi \in \Xi_{X}\right\} \subset \lambda_{X}+i \mathbb{R}
$$

is finite. Then for each $Y_{0} \in \mathfrak{a}_{Z}$ we have

$$
\operatorname{deg}\left(t \mapsto q_{v, \xi, w}\left(Y_{0}+t X\right)\right) \leqslant d_{X}
$$

for all $v \in V, \xi \in \Xi_{X}$ and $w \in \mathcal{W}$, and there exist $\lambda \in \Lambda, v \in V$ and $w \in \mathcal{W}$ such that

$$
\operatorname{deg}\left(t \mapsto \sum_{\substack{\xi \in \Xi \\ \xi(X)=\lambda}} e^{\xi\left(Y_{0}\right)} q_{v, \xi, w}\left(Y_{0}+t X\right)\right)=d_{X} .
$$

Proof. We start with a general remark. Given $Y \in \mathfrak{a}_{Z}$ and a differentiable function $f$ on $\mathfrak{a}_{Z}$, we let $Y f$ be the derivative of $f$ with respect to $Y$. Then the coefficients of $m_{v, \eta}$ and $m_{Y v, \eta}$ are related by

$$
e^{\xi} q_{Y v, \xi, w}=-Y\left[e^{\xi} q_{v, \xi, w}\right] .
$$

Let $\xi \in \Xi_{X}$ and $w \in \mathcal{W}$. For each $v \in V$ we consider

$$
e^{\xi(Y)} q_{v, \xi, w}(Y+t X)
$$

as an analytic function of $Y \in \mathfrak{a}_{Z}$ into the space of polynomials in $t$ of degree $\leqslant d$. The value of this function at $Y=0$ belongs to the subspace of polynomials of degree $\leqslant d_{X}$, and by (6.27) the same is valid for all its derivatives at zero. It follows that it has degree $\leqslant d_{X}$ for all $Y$. This proves (6.25).

Likewise, for $\lambda \in \Lambda$ and $w \in \mathcal{W}$ we consider for each $v \in V$

$$
e^{-t \lambda(X)} \sum_{\substack{\xi \in \Xi \\ \xi(X)=\lambda}} e^{\xi(Y+t X)} q_{v, \xi, w}(Y+t X)
$$

as an analytic function of $Y \in \mathfrak{a}_{Z}$ into the space of polynomials in $t$ of degree $\leqslant d_{X}$. If (6.26) fails to hold, then (6.28) is a polynomial in $t$ of degree $<d_{X}$ for every $\lambda, v$ and $w$, and for $Y=Y_{0}$.

Then, again by (6.27), this will be the case also for the derivatives at $Y=Y_{0}$, and hence (6.28) has degree $<d_{X}$ at every $Y$. By the linear independence of the involved exponential polynomials of $Y$, this implies that the individual terms $q_{v, \xi, w}(Y+t X)$ also must have degree $<d_{X}$ at every $Y$, and hence in particular at $Y=0$. A contradiction with the definition of $d_{X}$ is then reached. 
Proposition 6.13. Assume that $\eta \neq 0$. Let $Y_{0} \in \mathfrak{a}_{Z}^{-}$and $X \in \mathbf{S}\left(\mathfrak{a}_{Z}^{-}\right)$. Then, there exist $w \in \mathcal{W}, v \in V$, a constant $C=C_{Y_{0}, X}>0$, an $\varepsilon>0$ and a sequence $t_{1}<t_{2}<\ldots$ with $t_{n} \rightarrow \infty$ such that

$$
\left|m_{v, \eta_{w}}\left(\exp \left(Y_{0}+t X\right) \cdot z_{w}\right)\right| \geqslant C \mathbf{w}_{\eta}(\exp (t X)) \mathbf{d}_{\eta}(\exp (t X))
$$

for all $t \in\left(t_{n}-\varepsilon, t_{n}+\varepsilon\right)$ and all $n \in \mathbb{N}$.

Proof. Consider the function

$$
F_{v, w}(t):=\sum_{\xi \in \Xi_{X}} q_{v, \xi, w}\left(Y_{0}+t X\right) e^{\xi\left(Y_{0}+t X\right)} .
$$

As in the proof of Lemma 6.11, we have

$$
m_{v, \eta}\left(\exp \left(Y_{0}+t X\right) w \cdot z_{0}\right)=F_{v, w}(t)+o\left(e^{t \lambda_{X}}\right)
$$

as $t \rightarrow \infty$

Let $\Lambda \subset \lambda_{X}+i \mathbb{R}$ be as in Lemma 6.12. Then

$$
F_{v, w}(t)=e^{t \lambda_{X}} \sum_{\lambda \in \Lambda} e^{i t \operatorname{Im} \lambda} \sum_{\substack{\xi \in \Xi \\ \xi(X)=\lambda}} e^{\xi\left(Y_{0}\right)} q_{v, \xi, w}\left(Y_{0}+t X\right) .
$$

For a one-variable polynomial $p(t)=\sum_{j=0}^{N} a_{j} t^{j}$ we denote by $p(t)_{j}=a_{j}$ the $j$ th coefficient. With $v$ and $w$ as in the final conclusion of Lemma 6.12, we set

$$
c_{\lambda}:=\sum_{\substack{\xi \in \Xi \\ \xi(X)=\lambda}} e^{\xi\left(Y_{0}\right)} q_{v, \xi, w}\left(Y_{0}+t X\right) d_{X}
$$

for $\lambda \in \Lambda$, and note that then $c_{\lambda} \neq 0$ for some $\lambda$. If we let

$$
F_{v, w}^{\mathrm{top}}(t):=e^{t \lambda_{X}} t^{d_{X}} \sum_{\lambda \in \Lambda} c_{\lambda} e^{i t \operatorname{Im} \lambda}
$$

then it follows from Lemma 6.14 below that

$$
\limsup _{t \rightarrow \infty}\left|e^{-t \lambda_{X}} t^{-d_{X}} F_{v, w}^{\text {top }}(t)\right|^{2} \neq 0 .
$$

Moreover, since the derivative of $\sum_{\lambda \in \Lambda} c_{\lambda} e^{i t \operatorname{Im} \lambda}$ is bounded above, we deduce the existence of positive constants $C$ and $\varepsilon$, and a sequence $t_{1}<t_{2}<\ldots$ with $t_{n} \rightarrow \infty$ such that

$$
\left|F_{v, w}^{\mathrm{top}}(t)\right| \geqslant C e^{t \lambda_{X}} t^{d_{X}}
$$

for all $\left|t-t_{n}\right|<\varepsilon$. Now (6.29) follows from (6.30).

The following lemma is shown in [36, Appendix 4.A.1.2(1)].

Lemma 6.14. Let $u_{1}, \ldots, u_{m} \in \mathbb{R}$ be distinct real numbers and let $c_{1}, \ldots, c_{m} \in \mathbb{C}$. Then

$$
\sum_{j=1}^{m}\left|c_{j}\right|^{2} \leqslant \limsup _{s \rightarrow \infty}\left|\sum_{j=1}^{m} c_{j} e^{i u_{j} s}\right|^{2} \text {. }
$$




\section{Quantitative upper bounds for generalized matrix coefficients}

The goal of this section is to achieve more quantitative versions of (6.22) and (6.24) in which the $v$-dependent constant $C$ is replaced by a continuous norm on $V$. For this, we shall need to impose on $G / H$ that it is either absolutely spherical or wave-front.

A norm $p$ on the Harish-Chandra module $V$ is called $G$-continuous, provided that the Banach completion $V_{p}$ of $V$ defines a Banach representation of $G$ with $V_{p}^{K \text {-fin }} \simeq_{\mathfrak{g}, K} V$ (see $[4, \S 2.2])$. It is a consequence of the Casselman embedding theorem that $G$-continuous norms exist.

Fix a basis $X_{1}, \ldots, X_{n}$ of $\mathfrak{g}$. Given a $G$-continuous norm $p$ on $V$, we define the $k$ th Sobolev norm $p_{k}$ of $p$ by

$$
p_{k}(v):=\sum_{m_{1}+\ldots+m_{n} \leqslant k} p\left(X_{1}^{m_{1}} \cdot \ldots \cdot X_{n}^{m_{n}} v\right), \quad v \in V,
$$

and note that $p_{k}$ is $G$-continuous as well. The following two statements are variants of the Casselman-Wallach globalization theorem (see [4]):

- For any two $G$-continuous norms $p$ and $q$ one has $V_{p}^{\infty} \simeq V_{q}^{\infty}$.

- For any two $G$-continuous norms $p$ and $q$ there exist $k \in \mathbb{N}$ and $C>0$ such that $p \leqslant C q_{k}$.

We use the notation $V^{\infty}:=V_{p}^{\infty}$, for any $G$-continuous norm $p$, and call $V^{\infty}$ the Casselman-Wallach globalization of $V$.

\subsection{The absolutely spherical case}

In the following lemma we assume that the orbit set $P_{\min , \mathbb{C}} \backslash G_{\mathbb{C}} / H_{\mathbb{C}}$ is finite. The condition that $P_{\min } \backslash G / H$ is finite is equivalent to $G / H$ being real spherical (see [26]), but finiteness of $P_{\text {min, }, \mathbb{C}} \backslash G_{\mathbb{C}} / H_{\mathbb{C}}$ is known to be a stronger condition (see [31, Remark 7]). It is fulfilled for absolutely spherical spaces, since then $B_{\mathbb{C}} \backslash G_{\mathbb{C}} / H_{\mathbb{C}}$ is finite for every Borel subgroup $B_{\mathbb{C}}$.

Lemma 7.1. Suppose that $P_{\min , \mathbb{C}} \backslash G_{\mathbb{C}} / H_{\mathbb{C}}$ is finite. Then the space $\mathfrak{h} V^{\infty}$ is closed in $V^{\infty}$ for every Harish-Chandra module $V$. In particular, if $Z$ is absolutely spherical, then $\mathfrak{h}_{I} V^{\infty}$ is closed in $V^{\infty}$ for each boundary degeneration $\mathfrak{h}_{I}$ of $\mathfrak{h}$.

Proof. The first statement is a result from [1]. For the second statement, we observe that $Z_{I}$ is absolutely spherical whenever $Z$ is (see Remark 3.3).

TheOrem 7.2. Assume that $Z$ is absolutely spherical. Let $(V, \eta)$ be a spherical pair and $\Omega \subset G$ be a compact subset.

(1) For every $X \in \mathbf{S}\left(\mathfrak{a}_{Z}^{-}\right)$there exists a $G$-continuous norm $q$ such that

$$
\left|m_{v, \eta}\left(\omega \exp (t X) w \cdot z_{0}\right)\right| \leqslant q(v) e^{t \lambda_{X}} t^{d_{X}}, \quad t \geqslant 1,
$$


for every $v \in V^{\infty}, \omega \in \Omega$ and $w \in \mathcal{W}$.

(2) Let $\mu:=\sum_{\sigma \in S} \sigma \in \mathfrak{a}_{Z}^{*}$. Then for all $\varepsilon>0$ there exists a $G$-continuous norm $q$ such that

$$
\left|m_{v, \eta}\left(\omega a w \cdot z_{0}\right)\right| \leqslant q(v) a^{\Lambda_{V, \eta}-\varepsilon \mu}, \quad a \in A_{Z}^{-},
$$

for every $v \in V^{\infty}, \omega \in \Omega$ and $w \in \mathcal{W}$.

Proof. For a $G$-continuous norm and a compact subset $\Omega \subset G$, there exists a constant $C>0$ such that $p(\omega v) \leqslant C p(v)$ holds for all $\omega \in \Omega$ and all $v \in V^{\infty}$. In view of $m_{g v, \eta}(z)=$ $m_{v, \eta}\left(g^{-1} z\right)$ for $g \in G$, this reduces the statements in (1) and (2) to $\omega=\mathbf{1}$. Likewise, with (6.18) and Corollary 3.8, we can reduce to $w=\mathbf{1}$ by applying that case to $\eta_{w}$.

(1) Let $X \in \mathbf{S}\left(\mathfrak{a}_{Z}^{-}\right)$be given and let $I:=\{\sigma \in S: \sigma(X)=0\}$. Recall then that $X \in \mathfrak{a}_{I}$ and that $\mathfrak{a}_{I}$ normalizes $\mathfrak{h}_{I}$. We infer from Lemma 7.1 that $\mathfrak{h}_{I} V^{\infty} \subset V^{\infty}$ is closed. In particular, the dual of $U:=V^{\infty} / \mathfrak{h}_{I} V^{\infty}$ is $\left(V^{-\infty}\right)^{H_{I}}$ and hence finite-dimensional. Thus $U$ is a finite-dimensional $\mathfrak{a}_{I}$-module.

To simplify the exposition, we pretend that all root spaces $\mathfrak{g}^{\alpha}$ are 1-dimensional. Fix a basis of root vectors $\left(X_{-\alpha}\right)_{\alpha}$ of $\overline{\mathfrak{u}}$, and set $Y_{-\alpha}:=X_{-\alpha}+T_{I}\left(X_{-\alpha}\right) \in \mathfrak{h}_{I}$. Further, let $Y_{j}$ be a basis for $\mathfrak{l} \cap \mathfrak{h}$. Then, the $Y_{-\alpha}$ together with the $Y_{j}$ form a basis for the real spherical subalgebra $\mathfrak{h}_{I}$. Note that

$$
\operatorname{Ad}(a) Y_{-\alpha}=a^{-\alpha} Y_{-\alpha}, \quad a \in A_{I}
$$

From the fact that $\mathfrak{h}_{I} V^{\infty}$ is closed in $V^{\infty}$, we also infer the following. Let $p$ be a $G$-continuous norm on $V^{\infty}$. Then, by the open mapping theorem, there exists a $G$ continuous norm $q$ such that every $v \in \mathfrak{h}_{I} V^{\infty}$ admits a presentation

$$
v=\sum_{\alpha} Y_{-\alpha} u_{\alpha}+\sum_{j} Y_{j} u_{j},
$$

where $u_{j}, u_{\alpha} \in V^{\infty}$ and

$$
\sum_{\alpha} p\left(u_{\alpha}\right)+\sum_{j} p\left(u_{j}\right) \leqslant q(v)
$$

After these preliminaries, we now begin the proof. As in the beginning of the proof of [24, Theorem 3.2], we begin with a crude estimate. There exist a $G$-continuous norm $p$ and a constant $\mu \in \mathbb{R}$ such that

$$
\left|m_{v, \eta}\left(\exp (t X) \cdot z_{0}\right)\right| \leqslant e^{\mu t} p(v), \quad v \in V^{\infty} \text { and } t \geqslant 0 .
$$

Let $\lambda_{0}=\lambda_{0}(X)$ be the infimum of the set of all $\mu$ for which such an estimate is valid for some $G$-continuous norm $p$. 
In the first step we consider vectors $v \in \mathfrak{h}_{I} V^{\infty}$ and let $v=\sum_{\alpha} Y_{-\alpha} u_{\alpha}+\sum Y_{j} u_{j}$ be a presentation as above. Then we compute with (7.1), for all $a \in A_{I}$,

$$
m_{v, \eta}\left(a \cdot z_{0}\right)=\sum_{\alpha} \eta\left(a^{-1} Y_{-\alpha} u_{\alpha}\right)=\sum_{\alpha} a^{\alpha} \eta\left(Y_{-\alpha} a^{-1} u_{\alpha}\right) .
$$

Note that $Y_{-\alpha}+\sum_{\alpha+\beta \notin\langle I\rangle} X_{\alpha, \beta} \in \mathfrak{h}$. Hence

$$
m_{v, \eta}\left(a \cdot z_{0}\right)=-\sum_{\alpha} \sum_{\alpha+\beta \notin\langle I\rangle} a^{\alpha} \eta\left(X_{\alpha, \beta} a^{-1} u_{\alpha}\right)=-\sum_{\alpha} \sum_{\alpha+\beta \notin\langle I\rangle} a^{\alpha+\beta} \eta\left(a^{-1} X_{\alpha, \beta} u_{\alpha}\right) .
$$

Notice that $a^{\alpha+\beta} \leqslant 1$ if $a \in A_{Z}^{-}$. We now specialize to $a=\exp (t X)$ with $t \geqslant 0$. Let

$$
c=c(X):=\max _{\alpha+\beta \notin\langle I\rangle}(\alpha+\beta)(X),
$$

and note that $c<0$, as $X$ determines $I$.

Let $p_{1}$ be a first order Sobolev norm of $p$, and let $q_{1}$ be related to $p_{1}$ as in (7.2). Then, by (7.3) and (7.4),

$$
\left|m_{v, \eta}\left(\exp (t X) \cdot z_{0}\right)\right| \leqslant e^{t(c+\mu)} \sum_{\alpha, \beta} p\left(X_{\alpha, \beta} u_{\alpha}\right) \leqslant C e^{t(\mu+c)} \sum_{\alpha} p_{1}\left(u_{\alpha}\right) \leqslant C e^{t(\mu+c)} q_{1}(v),
$$

where $C>0$ is a constant determined from the coordinates of $X_{\alpha, \beta}$ in terms of the basis for $\mathfrak{g}$ used in the Sobolev norm.

This brings us to the following improved estimate for elements from $\mathfrak{h}_{I} V^{\infty}$. For each $p$ and $\mu$ satisfying (7.3) there exists a $G$-continuous norm $p^{\prime}$ such that

$$
\left|m_{v, \eta}(\exp (t X))\right| \leqslant e^{t(\mu+c)} p^{\prime}(v), \quad t \geqslant 0 \text { and } v \in \mathfrak{h}_{I} V^{\infty} .
$$

Let $\mathcal{E}=\mathcal{E}_{X}$ be the spectrum of $X$ on $U$. We write $U=\bigoplus_{\lambda \in \mathcal{E}} U[\lambda]$ for the decomposition into generalized $X$-eigenspaces, and correspondingly $u=\sum_{\lambda} u_{\lambda}$ for $u \in U$. For $v \in V^{\infty}$ let $[v]=v+\mathfrak{h}_{I} V^{\infty}$ be its equivalence class in $U$. Let

$$
V^{\infty}[\lambda]=\left\{v \in V^{\infty}:[v] \in U[\lambda]\right\}
$$

for $\lambda \in \mathcal{E}$.

Let $\lambda \in \mathcal{E}$ and $v \in V^{\infty}[\lambda]$. Define

$$
v_{0}:=v \quad \text { and } \quad v_{i}:=(X-\lambda) v_{i-1}, \quad i=1,2, \ldots
$$

Let $d$ be the last value of $i$ for which $\left[v_{i}\right] \neq 0$. Then $X$ is represented by a lower triangular Jordan matrix on the invariant subspace $\mathbb{C}^{d+1} \simeq \operatorname{span}_{\mathbb{C}}\left\{\left[v_{0}\right], \ldots,\left[v_{d}\right]\right\} \subset U[\lambda]$. We denote by $B$ the transpose of that matrix. It follows from the definition of $v_{i}$ that

$$
p\left(v_{i}\right) \leqslant C p_{i}(v), \quad i=0,1, \ldots,
$$


for all $v \in V^{\infty}$, with a constant $C>0$ which depends on the coordinates of $X-\lambda$ in terms of the basis for $\mathfrak{g}$ used in the Sobolev norm $p_{i}$.

Now consider the $\mathbb{C}^{d+1}$-valued functions

$$
F(t)=\left(m_{v_{0}, \eta}(\exp (t X)), \ldots, m_{v_{d}, \eta}(\exp (t X))\right)
$$

and

$$
R(t)=-\left(0, \ldots, 0, m_{w, \eta}(\exp (t X)),\right.
$$

where $w=v_{d+1} \in \mathfrak{h}_{I} V^{\infty}$. Then $F$ satisfies the ordinary differential equation

$$
F^{\prime}(t)=-B F(t)+R(t)
$$

The general solution formula then gives

$$
F(t)=e^{-t B} F(0)+e^{-t B} \int_{0}^{t} e^{s B} R(s) d s .
$$

From (7.6) and (7.7) we infer that for each $\mu>\lambda_{0}$ there exists a $G$-continuous norm $p$, independent of $v$, such that

$$
|F(t)| \leqslant p(v) \max \left\{(1+t)^{d} e^{-\operatorname{Re} \lambda t}, e^{(\mu+c / 2) t}\right\} .
$$

In particular, this estimate applies to $m_{v, \eta}$.

We conclude that for each pair $(p, \mu)$ satisfying (7.3), there exists a $G$-continuous norm $p^{\prime \prime}$ such that

$$
\left|m_{v, \eta}(\exp (t X))\right| \leqslant \max \left\{e^{-t \operatorname{Re} \lambda}(1+t)^{d}, e^{(\mu+c / 2) t}\right\} p^{\prime \prime}(v), \quad t \geqslant 0,
$$

for all $\lambda \in \mathcal{E}_{X}$ and all $v \in V^{\infty}[\lambda]$. Here $d+1$ is determined as the maximal possible length of a Jordan block of $X$ on $U[\lambda]$.

Before we move on, we recall the following standard fact from functional analysis. Let $E$ be a Fréchet space and $F \subset E$ be a closed subspace, then $E / F$ is a Fréchet space. Moreover, if $\left\{p^{n}\right\}_{n}$ is a sequence of semi-norms which define the topology on $E$, then $\left[p^{n}\right](v+F):=\inf _{w \in F} p^{n}(v+w)$ is a family of semi-norms which defines the topology on $E / F$.

Our previous discussion shows that for every $G$-continuous norm $p$ on $V$ there is a $G$-continuous norm $q$ such that for all $v \in V^{\infty}$ we find $v_{\lambda} \in V^{\infty}[\lambda]$ with $\left[v_{\lambda}\right]=[v]_{\lambda}$ and

$$
p\left(v_{\lambda}\right) \leqslant q(v), \quad \lambda \in \mathcal{E} .
$$


The continuity expressed by this will be used to combine estimates for $v \in V^{\infty}[\lambda]$ with different $\lambda \in \mathcal{E}$ into an estimate for all $v \in V^{\infty}$.

We shall now split $\mathcal{E}$ into the disjoint union

$$
\mathcal{E}=\mathcal{E}_{-} \cup \mathcal{E}_{0} \cup \mathcal{E}_{+}
$$

of elements $\lambda$ with $-\operatorname{Re} \lambda<\lambda_{0},-\operatorname{Re} \lambda=\lambda_{0}$ and $-\operatorname{Re} \lambda>\lambda_{0}$, respectively.

For $\lambda \in \mathcal{E}_{-}$we obtain from (7.9) that there exists a $G$-continuous norm $p$ such that

$$
\left|m_{v, \eta}(\exp (t X))\right| \leqslant e^{\nu t} p(v), \quad t \geqslant 0 \text { and } v \in V^{\infty}[\lambda]
$$

for some $\nu<\lambda_{0}$.

Let $\lambda \in \mathcal{E}_{+} \cup \mathcal{E}_{0}$ and let $\mu+\frac{1}{2} c<\lambda_{0}<\mu$. Then it follows from (7.6) that the integral $\int_{0}^{\infty} e^{s B} R(s) d s$ is absolutely convergent. Hence,

$$
c^{\infty}=c^{\infty}(v):=\lim _{t \rightarrow \infty} e^{t B} F(t)=F(0)+\int_{0}^{\infty} e^{s B} R(s) d s \in \mathbb{C}^{d+1}
$$

exists and satisfies $\left|c^{\infty}(v)\right| \leqslant p(v)$ for a $G$-continuous norm $p$. The solution formula (7.8) can then be rewritten as

$$
F(t)=e^{-t B} c^{\infty}-e^{-t B} \int_{t}^{\infty} e^{s B} R(s) d s
$$

Moreover, we also obtain from (7.6) that

$$
\left|e^{-t B} \int_{t}^{\infty} e^{s B} R(s) d s\right|=\left|\int_{0}^{\infty} e^{s B} R(s+t) d s\right| \leqslant C p(v) e^{(\mu+c / 2) t}
$$

for a constant $C>0$.

In particular, the estimate (7.13) applies to $\mid m_{v, \eta}\left(\exp (t X) \mid\right.$ when $c^{\infty}=0$ and yields

$$
\left|m_{v, \eta}(\exp (t X))\right| \leqslant e^{(\mu+c / 2) t} p(v), \quad t \geqslant 0 .
$$

It follows that $c^{\infty}$ cannot be zero for all $\lambda \in \mathcal{E}_{0} \cup \mathcal{E}_{+}$and all $v \in V^{\infty}[\lambda]$, as in that case we would reach from (7.11) and (7.14) a contradiction with the definition of $\lambda_{0}$.

Let now $v \in V^{\infty}[\lambda]$ be such that $c^{\infty} \neq 0$, say $c^{\infty}=\left(c_{0}^{\infty}, \ldots, c_{k}^{\infty}, 0, \ldots, 0\right)$ with $c_{k}^{\infty} \neq 0$. Then

$$
\left|e^{-t B} c^{\infty}\right| \sim c_{k}^{\infty} e^{-t \operatorname{Re} \lambda} \frac{t^{k}}{k !}
$$

for $t$ large. Hence, with (7.12) and (7.13), for all $v \in V^{\infty}[\lambda]$ we obtain

$$
\left|m_{v, \eta}(\exp (t X))\right| \leqslant e^{-t \operatorname{Re} \lambda}(1+t)^{d_{\lambda}} p(v), \quad t \geqslant 0 .
$$


Here $d_{\lambda}$ is the maximal possible value of $k$ among all $v \in V^{\infty}[\lambda]$. In addition, we have for some $v \in V^{\infty}[\lambda]$ a non-trivial lower bound

$$
\left|m_{v, \eta}(\exp (t X))\right| \geqslant C_{0} t^{d_{\lambda}} e^{-t \operatorname{Re} \lambda}
$$

for $C_{0}>0$ and $t$ sufficiently large. It follows that $-\operatorname{Re} \lambda \leqslant \lambda_{0}$, and hence $\lambda \in \mathcal{E}_{0}$. In particular, we conclude that (7.14) applies to all $\lambda \in \mathcal{E}_{+}$.

By combining (7.11) for $\lambda \in \mathcal{E}_{-},(7.14)$ or (7.15) for $\lambda \in \mathcal{E}_{0}$ and (7.14) for $\lambda \in \mathcal{E}_{+}$, and using (7.10), we see that

$$
\left|m_{v, \eta}(\exp (t X))\right| \leqslant e^{t \lambda_{0}}(1+t)^{d_{0}} p(v), \quad t \geqslant 0
$$

for all $v \in V^{\infty}$, where $d_{0}$ is the maximal value of $d_{\lambda}$ for $\lambda \in \mathcal{E}_{0}$. Moreover, by (7.16),

$$
\left|m_{v, \eta}(\exp (t X))\right| \geqslant C_{0} t^{d_{0}} e^{t \lambda_{0}}
$$

for some $v \in V^{\infty}$. We conclude from (6.24) that $C_{0} t^{d_{0}} e^{t \lambda_{0}} \leqslant C t^{d_{X}} e^{t \lambda_{X}}$, and thus (7.17) implies the statement in (1). Note that then $\lambda_{0}=\lambda_{X}$ by Proposition 6.13.

(2) Recall that $\left|m_{v, \eta}\left(a b w \cdot z_{0}\right)\right|=\left|m_{v, \eta}\left(a w \cdot z_{0}\right)\right| b^{\Lambda_{V, \eta}}$ for all $b \in A_{Z, E}, a \in A_{Z}$ and $w \in \mathcal{W}$. Hence we may assume that $\mathfrak{a}_{Z, E}=0$ for our purpose.

For $\delta>0$ and $I \nsubseteq S$ we set

$$
\left.\mathbf{S}_{\delta}\left(\mathfrak{a}_{Z}^{-} \cap \mathfrak{a}_{I}\right):=\left\{X \in \mathbf{S}\left(\mathfrak{a}_{Z}^{-}\right) \cap \mathfrak{a}_{I}:-\alpha(X) \geqslant \delta \text { for all } \alpha \in S \backslash I\right)\right\}
$$

Then, for each $I \neq S$ and each $\delta>0$, we obtain an estimate as in (1) with a $G$-continuous norm $q=q_{\delta}$ which is uniform for all $X \in \mathbf{S}_{\delta}\left(\mathfrak{a}_{Z}^{-} \cap \mathfrak{a}_{I}\right)$. This is because in the proof of (1) only the constant $c$ from (7.5) enters in the quantification of $q$. Indeed, $q$ was constructed from the initial estimate (7.3), which is valid for all $X$, in a number of steps each of which lowered the exponent $\mu$ by $\frac{1}{2} c$. In each step a new norm $p^{\prime}$ was constructed from a previous one $p$, and this construction can be done independently of $X$, as long as it remains in the bounded set $S\left(\mathfrak{a}_{Z}^{-}\right)$. Since the constant $\mu$ in the initial estimate (7.3) can be chosen independently of $X$, and since $\lambda_{0}(X)=\lambda_{X}$ is uniformly bounded below by continuity, the maximal number of steps depends only on $c$.

Further, for $|S \backslash I|=1$, i.e. $\mathfrak{a}_{I}$ is 1-dimensional, we note that $\mathbf{S}_{\delta}\left(\mathfrak{a}_{Z}^{-} \cap \mathfrak{a}_{I}\right)=\mathbf{S}\left(\mathfrak{a}_{Z}^{-}\right) \cap \mathfrak{a}_{I}$ if $\delta$ is small enough. Hence, in this case, we have a-priori estimates which are uniform for all $X \in \mathbf{S}\left(\mathfrak{a}_{Z}^{-}\right) \cap \mathfrak{a}_{I}$. The proof then proceeds by induction on $\operatorname{dim} \mathfrak{a}_{I}$. We explain the proof for the final step where $X \in \mathbf{S}\left(\mathfrak{a}_{Z}^{-}\right) \cap \mathfrak{a}_{I}$ for $\mathfrak{a}_{I}=\mathfrak{a}_{Z}$. By the induction hypothesis, we then have uniform estimates for all $X \in \mathbf{S}\left(\mathfrak{a}_{Z}^{-}\right) \cap \mathfrak{a}_{I}$ with $|I| \geqslant 1$.

Let $\delta>0$ be fixed. It will be described at the end of the proof how it is chosen from the given $\varepsilon$. As explained above, we have a uniform estimate for $X \in \mathbf{S}_{\delta}\left(\mathfrak{a}_{Z}^{-}\right)$. For the 
remaining $X \in \mathbf{S}\left(\mathfrak{a}_{Z}^{-}\right)$, we have $-\delta<\alpha(X) \leqslant 0$ for at least one $\alpha \in S$, and hence we can write $X=X_{1}+X_{2}$ where $X_{1} \in S\left(\mathfrak{a}_{Z}^{-}\right) \cap \mathfrak{a}_{I}$ for some $I \subset S$ with $|I| \geqslant 1$ and $X_{2} \in \mathfrak{a}_{Z}$ is small relative to $\delta$, i.e. $\left\|X_{2}\right\|<C \delta$ with a constant $C$ independent of $\delta$.

Observe that $m_{v, \eta}\left(\exp (t X) \cdot z_{0}\right)=m_{\exp \left(t X_{2}\right) v, \eta}\left(\exp \left(t X_{1}\right) \cdot z_{0}\right)$, and thus from (1) we obtain that, for $t \geqslant 1$,

$$
\left|m_{v, \eta}\left(\exp (t X) \cdot z_{0}\right)\right| \leqslant q\left(\exp \left(t X_{2}\right) v\right) e^{t \lambda_{X_{1}}} t^{d_{X_{1}}} .
$$

It follows from (6.23) that $\lambda_{X_{1}} \leqslant \Lambda_{V, \eta}\left(X_{1}\right)$. Since $q$ is $G$-continuous, we have

$$
q\left(\exp \left(t X_{2}\right) v\right) \leqslant e^{t\left\|X_{2}\right\| c_{q}} q(v)
$$

where $c_{q}>0$ is a constant depending on $q$. Hence (2) follows if

$$
\left|\Lambda_{V, \eta}\left(X_{2}\right)\right|+c_{q}\left\|X_{2}\right\|<-\varepsilon \mu(X),
$$

and this can be attained uniformly with a proper choice of $\delta$, since $-\mu$ is bounded below on $S\left(\mathfrak{a}_{Z}^{-}\right)$.

Remark 7.3. The proof of (1) shows that $\lambda_{X}$ is minus the real part of an eigenvalue of $X$ on the finite-dimensional $\mathfrak{a}_{I}$-module $U=V^{\infty} /\left(\mathfrak{h}_{I}\right)_{w} V^{\infty}$ for some $w \in \mathcal{W}$.

Remark 7.4. In the group case $Z=(G \times G) / G \simeq G$, the existence of the norm $q$ in Theorem $7.2(2)$ makes the statement considerably stronger than the corresponding result in [36, Theorem 4.3.5], because it implies a bound for matrix coefficients $m_{\tilde{v}, v}$ on $G$, which is simultaneously uniform with respect to the two smooth vectors $v$ and $\tilde{v}$. We refer to [24, Theorem 5.8], where the generalization is obtained for symmetric spaces and subsequently explicated for the group case.

\subsection{The wave-front case}

For this case, we replace the smooth globalization $V^{\infty}$ by the analytic globalization $V^{\omega} \subset V^{\infty}$. We briefly recall its construction (see [21, Remark 6.6]). Let $p$ be any $G$ continuous norm on $V$. Then there is a family of analytic norms (not necessarily $G$ continuous) $\left\{p_{\varepsilon}\right\}_{\varepsilon>0}$ on $V$, such that $V^{\omega}=\underline{\lim } V^{\varepsilon}$ with $V^{\varepsilon}$ the completion of $V$ with respect to $p_{\varepsilon}$. These norms feature the following properties:

(a) for $\varepsilon \leqslant \varepsilon^{\prime}$ one has $p_{\varepsilon} \leqslant p_{\varepsilon^{\prime}}$ and continuous inclusion $V^{\varepsilon^{\prime}} \rightarrow V^{\varepsilon}$;

(b) for each compact set $\Omega \subset G$ and $\varepsilon>0$ there exist $0<\varepsilon^{\prime} \leqslant \varepsilon$ and a constant $C$ such that $p_{\varepsilon^{\prime}}(g \cdot v) \leqslant C p_{\varepsilon}(v)$ for all $v \in V$ and $g \in \Omega$;

(c) for each $G$-continuous norm $q$ and $\varepsilon>0$, there exists a constant $C$ such that $q \leqslant C p_{\varepsilon}$. 
Let $F \subset \Pi$ and let $P_{F}=G_{F} U_{F}$ be the associated parabolic subgroup as described in $\S 4$. For any Harish-Chandra module $V$ we recall that $V / \overline{\mathfrak{u}}_{F} V$ is a Harish-Chandra module for the pair $\left(\mathfrak{g}_{F}, K_{F}\right)$, and as such has an analytic globalization $\left(V / \overline{\mathfrak{u}}_{F} V\right)^{\omega}$ as $G_{F}$-representation.

Lemma 7.5. Let $V$ be a Harish-Chandra module. Then $\overline{\mathfrak{u}}_{F} V^{\omega}$ is closed in $V^{\omega}$. In particular, $V^{\omega} / \overline{\mathfrak{u}}_{F} V^{\omega}=\left(V / \overline{\mathfrak{u}}_{F} V\right)^{\omega}$.

Proof. Since the analytic globalization $V^{\omega}$ coincides with the minimal globalization of Kashiwara-Schmid, this follows from the version of the Casselman comparison theorem established in [6, Theorem 1] or [9, Theorem 1.3].

TheOrem 7.6. Assume that $Z$ is wave-front. Let $V$ be a Harish-Chandra module, $(V, \eta)$ be a spherical pair, and $p$ be a G-continuous norm on $V$. Then there exists a constant $d \in \mathbb{N}$ such that the following holds for each compact subset $\Omega \subset G$ and each $X \in \mathbf{S}\left(\mathfrak{a}_{Z}^{-}\right)$: for every $\varepsilon>0$ there exists a constant $C>0$ such that

$$
\left|m_{v, \eta}\left(\omega a \exp (t X) w \cdot z_{0}\right)\right| \leqslant C p_{\varepsilon}(v) e^{t \lambda_{X}} t^{d_{X}} a^{\Lambda_{V, \eta}}(1+\|\log a\|)^{d}
$$

for all $v \in V, t \geqslant 1, a \in A_{Z}^{-}, \omega \in \Omega$ and $w \in \mathcal{W}$.

Proof. By using the property (b) for the norms $p_{\varepsilon}$, we reduce to $\omega=\mathbf{1}$ as in the proof of Theorem 7.2. Likewise we can assume that $w=1$.

Each element $X \in \mathbf{S}\left(\mathfrak{a}_{Z}^{-}\right)$determines a set $I:=\{\sigma \in S \mid \sigma(X)=0\}$. As we assume that $Z$ is wave-front we obtain a parabolic $\mathfrak{p}_{F}=\mathfrak{p}_{F_{I}}$ from Proposition 5.5. In particular, there exists an element $Y \in \mathfrak{a}^{-}$such that $Y+\mathfrak{a}_{H}=X$ and $F=\{\alpha \in \Pi \mid \alpha(Y)=0\}$. Moreover, for every $\sigma=\alpha+\beta \in \mathcal{M}$ with $\alpha \in \Sigma_{\mathfrak{u}} \backslash\langle F\rangle$ we record

$$
(\alpha+\beta)(X)=(\alpha+\beta)(Y)<0 .
$$

The proof is quite analogous to the one for Theorem 7.2(1). We confine ourselves to the steps where it differs. The main difference consists of replacing $U=V^{\infty} / \mathfrak{h}_{I} V^{\infty}$ by $U:=V^{\omega} / \overline{\mathfrak{u}}_{F} V^{\omega}$, and applying Lemma 7.5 in place of Lemma 7.1. We observe that as $U$ is a Harish-Chandra module for $\mathfrak{g}_{F}$, it admits a finite decomposition into generalized eigenspaces for $\mathfrak{a}_{F}$.

As before, we pretend that all root spaces $\mathfrak{g}^{\alpha}$ are 1-dimensional and fix a basis of root vectors $\left(X_{-\alpha}\right)_{\alpha}$ of $\overline{\mathfrak{u}}_{F}$. The expression (7.1) is just replaced by the corresponding expression for $\operatorname{Ad}(a) X_{-\alpha}$, where $a \in A$.

The inequality (7.2) has the following analytic counterpart. For all $\varepsilon>0$ there exist $\varepsilon^{\prime}>0$ and a constant $C_{\varepsilon}>0$ such that every $v \in \overline{\mathfrak{u}}_{F} V$ admits a presentation $v=\sum_{\alpha} X_{-\alpha} u_{\alpha}$, with $u_{\alpha} \in V$ and

$$
\sum_{\alpha} p_{\varepsilon^{\prime}}\left(u_{\alpha}\right) \leqslant C_{\varepsilon} p_{\varepsilon}(v)
$$


This follows from Lemma 7.5, by the argument of [21, Remark 6.6] (note that $\varepsilon$ and $\varepsilon^{\prime}$ need to be interchanged in (6.5) of that remark).

We replace the initial estimate (7.3) by the following, which is derived from it with property (c) for the norms $p_{\varepsilon}$. For some $\lambda \in \mathfrak{a}_{Z}^{*}$, some $\mu \in \mathbb{R}$ and all $\varepsilon^{\prime}>0$ there exist a constant $C$ such that

$$
\left|m_{v, \eta}\left(a \exp (t X) \cdot z_{0}\right)\right| \leqslant C e^{t \mu} a^{\lambda} p_{\varepsilon^{\prime}}(v), \quad t \geqslant 0 \text { and } a \in A_{Z}^{-}
$$

Let $v \in \overline{\mathfrak{u}}_{F} V$. With (3.3) and the above presentation of $v$ we obtain, as in (7.4),

$$
m_{v, \eta}\left(a \cdot z_{0}\right)=-\sum_{\alpha} \sum_{\beta} a^{\alpha+\beta} \eta\left(a^{-1} X_{\alpha, \beta} u_{\alpha}\right)
$$

for all $a \in A_{Z}$. Here $\alpha+\beta \in \mathcal{M}$, and hence $a^{\alpha+\beta} \leqslant 1$ if $a \in A_{Z}^{-}$. In addition $\alpha \notin\langle F\rangle$, and hence, by (7.18), there exists $c<0$ such that $(\alpha+\beta)(X) \leqslant c$ for all terms in (7.21).

From the crude estimate (7.20) we then obtain

$$
\left|m_{v, \eta}\left(a \exp (t X) \cdot z_{0}\right)\right| \leqslant C a^{\lambda} e^{t(\mu+c)} \sum_{\alpha, \beta} p_{\varepsilon^{\prime}}\left(X_{\alpha, \beta} u_{\alpha}\right), \quad t \geqslant 0 \text { and } a \in A_{Z}^{-} .
$$

Since $p_{\varepsilon^{\prime}}$ is analytic, there exists $C^{\prime}>0$ such that $p_{\varepsilon^{\prime}}\left(X_{\alpha, \beta} u\right) \leqslant C^{\prime} p_{\varepsilon^{\prime}}(u)$ for all $u \in V$, and from (7.19) we finally derive the following improved estimate, analoguous to (7.6).

For each $\mu$ satisfying (7.20) and for each $\varepsilon>0$ there exists a constant $C$ such that

$$
\left|m_{v, \eta}\left(a \exp (t X) \cdot z_{0}\right)\right| \leqslant C a^{\lambda} e^{t(\mu+c)} p_{\varepsilon}(v), \quad t \geqslant 0 \text { and } a \in A_{Z}^{-}
$$

for all $v \in \overline{\mathfrak{u}}_{F} V$.

Following the previous proof, with $\mathcal{E}=\mathcal{E}_{X}$ now denoting the spectrum of $X$ on the $\mathfrak{a}_{F}$-finite module $U=V / \overline{\mathfrak{u}}_{F} V$, we arrive at the bound

$$
\left|m_{v, \eta}\left(a \exp (t X) \cdot z_{0}\right)\right| \leqslant C p_{\varepsilon}(v) a^{\lambda} e^{t \lambda_{X}} t^{d_{X}}
$$

for all $t \geqslant 1, \omega \in \Omega, w \in \mathcal{W}$ and $a \in A_{Z}^{-}$. Using the coordinates from (5.1) for $\mathfrak{a}_{Z}^{-}$, we can now iterate with the sequence $X=-\omega_{1}, \ldots,-\omega_{s}$ and optimize $\lambda$ to $\Lambda_{V, \eta}$ at the cost of a possible logarithmic term.

\section{Discrete series}

All $\eta \in\left(V^{-\infty}\right)^{H}$ to be considered in the sequel are requested to be $A_{Z, E}$-eigenvectors, say $a \cdot \eta=a^{\chi} \eta$. Recall from (6.21) that then $\left.\Lambda_{V, \eta}\right|_{\mathfrak{a}_{Z, E}}=\operatorname{Re} \chi$. 
For $Z$ unimodular and for an irreducible Harish-Chandra module $V$ we introduced in [21, Definition 5.3] the notion of a $Z$-tempered pair $(V, \eta)$, and showed in [21, Proposition 5.5] that this condition is satisfied by almost all pairs that contribute to the Plancherel decomposition of $Z$. With [21] Remark $5.4(V, \eta)$ is $Z$-tempered if and only if for all $v \in V$ there exists a constant $C_{v}>0$ and an $m \in \mathbb{N}$ such that

$$
\left|m_{v, \eta}\left(\omega a w \cdot z_{0}\right)\right| \leqslant C_{v} a^{\varrho_{Q}}(1+\|\log a\|)^{m}
$$

for all $\omega \in \Omega, w \in \mathcal{W}$ and $a \in A_{Z}^{-}$. Here $\varrho_{Q}:=\varrho_{\mathfrak{u}} \in \mathfrak{a}_{Z}^{*}$ is defined by $2 \varrho_{Q}(X)=\operatorname{tr} \operatorname{ad}_{\mathfrak{u}}(X)$, and $\Omega$ denotes a compact set for which (3.16) is valid.

Remark 8.1. In (8.1) we can replace the constant $C_{v}$ by $q(v)$, where $q$ is a $G$ continuous norm (see [21, Definition 5.3]). Hence the notion of a tempered pair $(V, \eta)$ only depends on $V^{\infty}$ and not on the particular choice of the maximal compact subgroup $K$.

We now give a criterion for temperedness in terms of $\Lambda_{V, \eta}$. Let us first note that, by (6.4) and (6.21), the temperedness (8.1) implies

$$
\left.\left(\Lambda_{V, \eta}-\varrho_{Q}\right)\right|_{\mathfrak{a}_{Z, E}}=0 .
$$

THEOREM 8.2. Let $Z$ be a unimodular real spherical space and $(V, \eta)$ be an $H$ spherical pair. Then (1) implies (2) for the following assertions:

(1) $(V, \eta)$ is tempered;

(2) $\left.\left(\Lambda_{V, \eta}-\varrho_{Q}\right)\right|_{\mathfrak{a}_{Z}^{-}} \leqslant 0$.

Moreover, if $Z$ is wave-front, (1) and (2) are equivalent.

Proof. It is immediate from Theorem 7.6 that (2) implies (1) when $Z$ is wave-front. For the converse we use the lower bound in Proposition 6.13 with $Y_{0}=0$ and $X=-\omega_{j}$, together with (8.2) and

$$
\lambda_{-\omega_{j}}=\Lambda_{V, \eta}\left(-\omega_{j}\right), \quad 1 \leqslant j \leqslant s .
$$

Recall the coordinates (5.1) of $\mathfrak{a}_{Z}$. In the context of Theorem 8.2, we shall say that the pair $(V, \eta)$ satisfies the strong inequality if

$$
\left(\Lambda_{V, \eta}-\varrho_{Q}\right)\left(\omega_{j}\right)>0, \quad 1 \leqslant j \leqslant s,
$$

holds, together with (8.2). This terminology is motivated by the relation of (8.3) to square integrability, which will be shown in Theorem 8.5. For that we need to recall some results on half-densities.

Remark 8.3. The strong inequality implies temperedness for spaces which are absolutely spherical or wave-front. In the latter case this is part of Theorem 8.2. Using Theorem 7.2 instead of Theorem 7.6 in the above proof, the assertion follows in the absolutely spherical case as well. 


\subsection{Densities on a homogeneous space}

In this section we consider a closed subgroup $H$ of a Lie group $G$ and the associated homogeneous space $Z=G / H$. In (3.26) we attached to $Z$ the modular character $\Delta_{Z}=$ $\left|\operatorname{det} \operatorname{Ad}_{\mathfrak{g} / \mathfrak{h}}\right|^{-1}$ on $H$.

Let $p=\operatorname{dim} Z$, fix $0 \neq \Omega \in \bigwedge^{p}(\mathfrak{g} / \mathfrak{h})^{*}$ and observe that

$$
\operatorname{Ad}^{*}(h)|\Omega|=\Delta_{Z}(h)|\Omega| \text {. }
$$

We view $\Lambda^{p}(\mathfrak{g} / \mathfrak{h})^{*}$ as a subspace of $\Lambda^{p} \mathfrak{g}^{*}$ and extend $\Omega$ to a $G$-invariant differential $p$-form on $G$ via

$$
\Omega(g)\left(d \lambda_{g}(\mathbf{1}) X_{1}, \ldots, d \lambda_{g}(\mathbf{1}) X_{p}\right)=\Omega\left(X_{1}, \ldots, X_{p}\right)
$$

for $X_{1}, \ldots, X_{p} \in \mathfrak{g}$ and with $\lambda_{g}(x)=g x$ for $x \in G$, the left displacement by $g$ on $G$.

Given a character $\xi$ of $H$, we set

$$
C^{\infty}(G ; \xi):=\left\{f \in C^{\infty}(G): f(\cdot h)=\xi(h)^{-1} f(\cdot) \text { for } h \in H\right\} .
$$

We write $C_{c}^{\infty}(G ; \xi)$ for the subspace of $C^{\infty}(G ; \xi)$ which consists of functions which are compactly supported modulo $H$.

Now observe that, if $f \in C^{\infty}\left(G ; \Delta_{Z}\right)$, then the density

$$
|\Omega|_{f}(g)\left(d \lambda_{g}(\mathbf{1}) X_{1}, \ldots, d \lambda_{g}(\mathbf{1}) X_{p}\right)=f(g)|\Omega|\left(X_{1}, \ldots, X_{p}\right)
$$

factors to a smooth density on $Z$ and every smooth density on $Z$ is of this type. In other words there is a natural identification

$$
\begin{aligned}
C^{\infty}\left(G ; \Delta_{Z}\right) & \longrightarrow \operatorname{Dens}^{\infty}(Z), \\
f & \longmapsto|\Omega|_{f},
\end{aligned}
$$

with the space Dens ${ }^{\infty}(Z)$ of smooth densities of $Z$. This identification is observed to be $G$-equivariant.

In a moment we shall need certain spaces of smooth half-densities, which are defined as follows. A character $\xi: H \rightarrow \mathbb{C}^{*}$ for which

$$
|\xi|=\Delta_{Z}^{1 / 2}
$$

will be called a normalized unitary character. For such characters we define on $C_{c}^{\infty}(G ; \xi)$ by

$$
\langle f, h\rangle:=\int_{Z}|\Omega|_{f \bar{h}}
$$

an inner product, which is preserved by the left regular action of $G$. We denote by $L^{2}(Z ; \xi)$ the Hilbert completion of $C_{c}^{\infty}(G ; \xi)$ with respect to this inner product, and note that it is a unitary $G$-module for the left regular representation. With the terminology of [29], it is the representation induced by the unitary character $\Delta_{Z}^{-1 / 2} \xi$ of $H$.

With that, we can define the $\xi$-twisted discrete series for $G / H$ as the set of (equivalence classes of) irreducible representations of $G$ which embed into $L^{2}(Z ; \xi)$. 


\subsection{A criterion for discrete series}

Let us return to the set-up from before $\S 8.1$, with $Z=G / H$ being real spherical and unimodular. By a slight change from previous notation, we denote in this section by $\widehat{H}$ the connected Lie subgroup of $G$ with Lie algebra $\hat{\mathfrak{h}}$. Likewise we set $\widehat{Z}=G / \widehat{H}$. Unlike $Z$, the space $\widehat{Z}$ is not necessarily unimodular. We write $\hat{\Delta}$ for the modular function of $\widehat{Z}$.

Lemma 8.4. Let $h a \in \widehat{H}$, with $a \in A_{Z, E}$ and $h \in H$. Then

$$
\hat{\Delta}(h a)=a^{-2 \varrho_{Q}} \text {. }
$$

Proof. Since $Z$ is unimodular and $H$ is a normal subgroup of $\widehat{H}$, we deduce that $H \subset \operatorname{ker} \hat{\Delta}$. Hence

$$
\hat{\Delta}(h a)=\left|\operatorname{det} \operatorname{Ad}_{\mathfrak{g} / \hat{\mathfrak{h}}}(a)\right|^{-1} .
$$

Finally observe that $\mathfrak{h}$ is isomorphic to $(\mathfrak{l} \cap \mathfrak{h})+\overline{\mathfrak{u}}$ as an $A_{Z, E}$-module. The assertion follows.

We extend the character $e^{-\chi}$ of $A_{Z, E}$ to a character $\xi$ of $\widehat{H}$, which is trivial on $H$, and note that it then follows from $a \cdot \eta=e^{\chi} \eta$ that $m_{v, \eta} \in C^{\infty}(\widehat{Z} ; \xi)$. It follows from Lemma 8.4 that $\xi$ is normalized unitary if and only if $\operatorname{Re} \chi=\varrho_{Q}$ (compare also (6.2)).

THEOREM 8.5. Let $Z$ be unimodular, $V$ be a Harish-Chandra module and $\eta \in\left(V^{-\infty}\right)^{H}$ be an element with eigencharacter $e^{\chi}$ on $A_{Z, E}$. Let $\xi=e^{-\chi}$. Then (i) implies (ii) for the following assertions:

(i) $\xi$ is normalized unitary and $m_{v, \eta} \in L^{2}(\widehat{Z} ; \xi)$ for all $v \in V$;

(ii) $(V, \eta)$ satisfies the strong inequality (see (8.3)),

Moreover, if $Z$ is absolutely spherical or wave-front, (i) and (ii) are equivalent.

It is a consequence of (i) that (the completion of) $V$ belongs to the $\xi$-twisted discrete series of $\widehat{Z}$.

Proof. Assume (ii). It follows from (8.2) that $\operatorname{Re} \chi=\Lambda_{V, \eta}=\varrho_{Q}$ on $\mathfrak{a}_{Z, E}$. Hence $\xi$ is normalized unitary and $|\Omega|_{v}:=|\Omega|_{\left|m_{v, \eta}\right|^{2}}$ is a density on $\widehat{Z}$ for each $v \in V$. We need to show that it is integrable. Recall from (3.19), applied to $\widehat{Z}=G / \widehat{H}$, that

$$
\widehat{Z}=\mathcal{F}^{\prime \prime} K A_{\widehat{Z}}^{-} \mathcal{W} \cdot \hat{z}_{0},
$$

where $\mathcal{F}^{\prime \prime}$ is a finite set. Keep in mind that $A_{\widehat{Z}}^{-}=A_{Z}^{-} / A_{Z, E}$ and that we realize $A_{\widehat{Z}} \subset A_{Z}$. Let $M_{H}=\left[Z_{K \cap H}\left(A_{\widehat{Z}}\right)\right]_{e}$ and note that $M_{H} \mathcal{W} \subset \mathcal{W} \hat{H}$. Hence, the the polar map

$$
\Phi: \mathcal{F}^{\prime \prime} \times K / M_{H} \times A_{\widehat{Z}}^{-} \times \mathcal{W} \longrightarrow \widehat{Z}
$$

associated with (8.5) is defined. We note that

- $\Phi$ has generically invertible differential;

- $\Phi$ has generically finite fibers, 
and that the second item follows from the first, as $\Phi$ is, up to finite cover, the restriction of a dominant algebraic map between complex varieties of the same dimension. Let $|\omega|_{v}:=\Phi^{*}\left(|\Omega|_{v}\right)$ be the pull-back of the density $|\Omega|_{v}$. It follows from the observations above that $|\omega|_{v}$ is integrable if and only if $|\Omega|_{v}$ is. Let us now compute this pull-back explicitely. For a subspace $U \subset \mathfrak{g}$ we fix a basis $u_{1}, \ldots, u_{p}$ and set $\omega_{U}=u_{1} \wedge \ldots \wedge u_{p} \in \bigwedge^{p} \mathfrak{g}$. Let $U$ be the orthogonal complement of $\mathfrak{m}_{H}$ in $\mathfrak{k}$. Define a function $J$ on $A_{\widehat{Z}}$ by

$$
J(a)\left|\omega_{\mathfrak{g}}\right|=\left|\omega_{U} \wedge \omega_{\mathfrak{a}_{\widehat{Z}}} \wedge\left[\operatorname{Ad}(a) \omega_{\hat{\mathfrak{h}}}\right]\right| .
$$

Then there exists a constant $C>0$ (depending on the normalization of measures) such that

$$
|\omega|_{v}\left(w^{\prime}, k M_{H}, a, w\right)=C\left|m_{v, \eta}\left(w^{\prime} k a w \cdot z_{0}\right)\right|^{2} J(a)|d|(a)|d|\left(k M_{H}\right)
$$

for $w^{\prime} \in \mathcal{F}^{\prime \prime}$ and $w \in \mathcal{W}$, where $|d|\left(k M_{H}\right)$ and $|d|(a)$ are the Haar densities on $K / M_{H}$ and $A_{\widehat{Z}}$, respectively. We deduce from (8.6) and (3.11) that $J(a) \leqslant C a^{-2 \varrho Q}$ for all $a \in A_{\widehat{Z}}^{-}$.

If $Z$ is absolutely spherical or wave-front, the integrability of $|\omega|_{v}$ now follows from Theorem 7.2 (2) and Theorem 7.6. Hence (i) is valid in that case. Assume (i). We argue by contradiction and assume that (8.3) fails, i.e. there exists $\omega_{j}$ such that

$$
\left(\Lambda_{V, \eta}-\varrho_{Q}\right)\left(\omega_{j}\right) \leqslant 0 .
$$

It suffices to show that $|\Omega|_{v}$ is not integrable for some $v$, when pulled back to $K \times A_{\widehat{Z}}^{-}$as in the first part of the proof. Suppose that $v \in V$ generates an irreducible $K$-module $V_{\tau}$ with dimension $d_{\tau}$. Then Schur-orthogonality implies that

$$
\int_{K / M_{H}}\left|m_{v, \eta}\left(k a w \cdot z_{0}\right)\right|^{2} d\left(k M_{H}\right) \geqslant \frac{1}{d_{\tau}}\left|m_{v, \eta}\left(a w \cdot z_{0}\right)\right|^{2},
$$

and therefore

$$
\int\left|\omega_{v}\right| \geqslant \frac{C}{d_{\tau}} \int_{A_{\bar{Z}}^{-}}\left|m_{v, \eta}\left(a w \cdot z_{0}\right)\right|^{2} J(a) d a .
$$

Next, we need a lower bound on the Jacobian function $J(a)$. We employ again (8.6) and (3.11), and deduce for $a_{0} \in A_{\widehat{Z}}^{-}$sufficiently far from walls that

$$
J\left(a_{0} \exp \left(-t \omega_{j}\right)\right) \geqslant C e^{2 t \varrho_{Q}\left(\omega_{j}\right)}
$$

for all $t \geqslant 0$ and a positive constant $C$. Hence it follows from (8.7), Proposition 6.13 and the Fubini theorem that $|\Omega|_{v}$ is not square integrable for some $v$.

Remark 8.6. In case $Z$ is wave-front or absolutely spherical, the above theorem shows that if $(V, \eta)$ satisfies the strong inequality, then this is also the case if we replace $V$ by the space of $K$-fixed vectors in $V^{\infty}$ for any other regular choice of $K$. 


\subsection{The interlaced case}

Recall that $H$ is said to be interlaced by $P$ if $U_{P} \subset H \subset P$, and that in this case $Z_{P}=$ $G_{P} / H_{P} \simeq P / H$ for the Levi-induced spherical space.

Lemma 8.7. If $H$ is interlaced by $P$ and $V$ embeds into $L^{2}(Z, \xi)$ for some normalized unitary character $\xi$ with trivial restriction to $U_{P}$, then there exists a twisted discrete series $\sigma$ of $Z_{P}$ such that $V$ embeds into the representation of $G$ unitarily induced from $\sigma \otimes 1$ of $P=G_{P} U_{P}$.

Proof. We may assume that $V$ is irreducible. Note that $L^{2}(Z, \xi)$ is unitary equivalent with the induced representation $\operatorname{Ind}_{H}^{G}\left(\xi \otimes \Delta_{Z}^{-1 / 2}\right)$. Induction by stages (see [29]) implies that this is unitary equivalent with $\operatorname{Ind}_{P}^{G} \operatorname{Ind}_{H}^{P}\left(\xi \otimes \Delta_{Z}^{-1 / 2}\right)$. Since $\xi$ is trivial on $U_{P}$, the representations $\operatorname{Ind}_{H}^{P}\left(\xi \otimes \Delta_{Z}^{-1 / 2}\right)$ and $\operatorname{Ind}_{H_{P}}^{G_{P}}\left(\left.\xi\right|_{H_{P}} \otimes \Delta_{Z}^{-1 / 2}\right) \otimes 1$ of $P=G_{P} U_{P}$ are unitary equivalent. Let

$$
\xi_{P}=\left.\xi\right|_{H_{P}} \otimes \Delta_{Z}^{-1 / 2} \otimes \Delta_{Z_{P}}^{1 / 2}
$$

Then $L^{2}\left(Z_{P}, \xi_{P}\right) \simeq \operatorname{Ind}_{H_{P}}^{G_{P}}\left(\xi \otimes \Delta_{Z}^{-1 / 2}\right)$. Hence $V$ embeds into the unitary representation $\operatorname{Ind}_{P}^{G}\left(L^{2}\left(Z_{P}, \xi_{P}\right) \otimes 1\right)$, and this implies that it embeds in $\operatorname{Ind}_{P}^{G}(\sigma \otimes 1)$ for some irreducible subrepresentation $\sigma$ of $L^{2}\left(Z_{P}, \xi_{P}\right)$.

\section{The tempered spectrum of $Z$}

Let $(V, \eta)$ be a spherical pair. In this section we use the power series expansions of $m_{v, \eta}$ from $\S 6$ to define maps

$$
\begin{gathered}
\left(V^{-\infty}\right)^{H} \longrightarrow\left(V^{-\infty}\right)^{\left(H_{w}\right)_{I}} \\
\eta \longmapsto \eta_{I}^{\mu, w}
\end{gathered}
$$

where $\mu$ runs through certain elements of $\mathcal{E}$, the leading exponents of the $m_{v, \eta}$. These maps can be thought of as Radon transforms for a single spherical representationanalytically a Radon transform would be the map which takes a rapidly decaying function on $G / H$ to a function on $G /\left(H_{w}\right)_{I}$ by integrating out fibers $\left(H_{w}\right)_{I} /\left(H \cap\left(H_{w}\right)_{I}\right)$. The coefficients of the power series expansion of $m_{v, \eta}$ are indexed by $\mathcal{E} \times \mathbb{N}_{0}[S]$. Roughly speaking, discarding all the coefficients not belonging to $\{\mu\} \times \mathbb{N}_{0}[I]$ and evaluating the partial expansion at $w \in \mathcal{W}$ produces an $\left(\mathfrak{h}_{w}\right)_{I}$-invariant linear functional $\eta_{I}^{\mu, w}$ on $V$ (see Lemma 9.2). The difficult part is then to see that $\eta_{I}^{\mu, w}$ is continuous, i.e. extends continuously to $V^{\infty}$. Under the assumption that $Z$ is wave-front or absolutely spherical, this is achieved in Lemma 9.4 using the sharp bounds from $\S 7$. Since $A_{I}$ acts on the finitedimensional space $\left(V^{-\infty}\right)^{\left(\mathfrak{h}_{w}\right)_{I}}$, we obtain a finite-dimensional $A_{I}$-module $W$-generated 
by $\eta_{I}^{\mu, w}$. Appropriate $A_{I}$-eigenvectors which we call $\eta$-optimal, then give the tempered embedding theorem (Theorem 9.11) for a tempered pair $(V, \eta)$.

\subsection{Leading coefficient maps}

Let $(V, \eta)$ be an $H$-spherical pair as considered in $\S 6$, and let $\Xi \subset \mathfrak{a}_{Z, \mathbb{C}}^{*}$ be the associated set of exponents (see (6.15)). We shall attach a set of leading coefficient maps

$$
\mathcal{L}_{I}: V \longrightarrow C^{\infty}\left(U_{A} A_{Z}^{-}\right)
$$

to each subset $I \subset S$.

Let $\Xi_{I}=\left\{\left.\xi\right|_{\mathfrak{a}_{I}}: \xi \in \Xi\right\}$ be the set of exponents along $A_{I}$. In analogy with $\mathcal{E}_{\text {lead }}$, we let $\mathcal{E}_{\text {lead }, I}$ consist of those exponents $\mu \in \Xi_{I}$ for which $\mu-\left.\sigma\right|_{\mathfrak{a}_{I}} \notin \Xi_{I}$ for all $\sigma \in \mathbb{N}_{0}[S] \backslash\langle I\rangle$ (see $(6.16))$.

Then

$$
\varnothing \neq\left.\mathcal{E}_{\text {lead }, I} \subset \mathcal{E}_{\text {lead }}\right|_{\mathfrak{a}_{I}}
$$

and

$$
\Xi_{I} \subset \mathcal{E}_{\text {lead }, I}+\left.\mathbb{N}_{0}[S]\right|_{\mathfrak{a}_{I}}=\mathcal{E}_{\text {lead }, I}+\mathbb{N}_{0}\left[\left.(S \backslash I)\right|_{\mathfrak{a}_{I}}\right] .
$$

For each $\mu \in \mathcal{E}_{\text {lead }, I}$ and each $w \in \mathcal{W}$ we define

$$
\mathcal{L}_{I}^{\mu, w}(v)(a):=\sum_{\substack{\left.\xi \in \Xi \\ \xi\right|_{\mathfrak{a}_{I}}=\mu}} q_{v, \xi, w}(\log a) a^{\xi}
$$

for $v \in V$ and $a \in U_{A} A_{Z}^{-}$, where $q_{v, \xi, w}$ is defined by (6.17).

Lemma 9.1. Let $\mu \in \mathcal{E}_{\text {lead }, I}$ and $w \in \mathcal{W}$. The linear map $\mathcal{L}_{I}^{\mu, w}: V \rightarrow C^{\infty}\left(U_{A} A_{Z}^{-}\right)$is $\mathfrak{a}_{Z}$-equivariant.

Proof. This follows from the uniqueness of the coefficients in the asymptotic expansions of $m_{v, \eta}\left(a w \cdot z_{0}\right)$ and $\mathcal{L}_{I}^{\mu, w}(v)(a)$ (see also (6.27)).

Denote by $V^{*}$ the algebraic dual of $V$ and define $\mathbf{L}_{I}^{\mu, w}: A_{I} \rightarrow V^{*}$ by

$$
\mathbf{L}_{I}^{\mu, w}(a)(v):=\mathcal{L}_{I}^{\mu, w}(v)(a) .
$$

Notice that

$$
\mathbf{L}_{I}^{\mu, w}(a)(v)=a^{\mu} p_{v, \mu, w}(\log a), \quad a \in A_{I},
$$

for a polynomial $p_{v, \mu, w}$ on $\mathfrak{a}_{I}$.

Lemma 9.2. Let $\mu \in \mathcal{E}_{\text {lead }, I}$ and $w \in \mathcal{W}$. Then $\operatorname{im} \mathbf{L}_{I}^{\mu, w} \subset\left(V^{*}\right)^{\left(\mathfrak{h}_{w}\right)_{I}}$. 
Proof. For the proof we assume first that $w=\mathbf{1}$ is trivial. Recall that, for the linear operator $T_{I}: \overline{\mathfrak{u}} \rightarrow(\mathfrak{l} \cap \mathfrak{h})^{\perp_{\mathfrak{l}}} \oplus \mathfrak{u}$ given by $(3.10)$,

$$
\mathfrak{h}_{I}=(\mathfrak{l} \cap \mathfrak{h})+\left\{\bar{X}+T_{I}(\bar{X}): \bar{X} \in \overline{\mathfrak{u}}\right\} .
$$

First, it is clear that $\left.m_{X v, \eta}\right|_{A_{Z}^{-}}=0$ for all $X \in \mathfrak{h} \cap \mathfrak{l}=\mathfrak{h}_{I} \cap \mathfrak{l}$. This readily implies that $\mathbf{L}_{I}^{\mu, \mathbf{1}}(a) \in\left(V^{*}\right)^{\mathfrak{l} \cap \mathfrak{h}}$ for all $a \in A_{I}$. Next, for $\alpha \in \Sigma_{\mathfrak{u}}$ and $X_{-\alpha} \in \mathfrak{g}^{-\alpha}$, we note that

$$
T\left(X_{-\alpha}\right)-T_{I}\left(X_{-\alpha}\right)=\sum_{\alpha+\beta \notin\langle I\rangle} X_{\alpha, \beta},
$$

with the notation of (3.3) and (3.10).

Let $X=X_{-\alpha}+T_{I}\left(X_{-\alpha}\right) \in \mathfrak{h}_{I}$ and note that, since $T_{I}\left(X_{-\alpha}\right)$ is a sum of root vectors $X_{\alpha, \beta} \in \mathfrak{g}^{\beta}$ with $\alpha+\beta \in\langle I\rangle$, we have $\operatorname{Ad}(a) X=a^{-\alpha} X$ for $a \in A_{I}$. Hence, for $v \in V$,

$$
m_{X \cdot v, \eta}\left(a \cdot z_{0}\right)=\eta\left(a^{-1} \cdot X \cdot v\right)=a^{\alpha} \eta\left(X a^{-1} \cdot v\right) .
$$

Let $Y=T\left(X_{-\alpha}\right)-T_{I}\left(X_{-\alpha}\right) \in \mathfrak{u}$. Then $X+Y \in \mathfrak{h}$, and for $a \in A_{I}$ we obtain from above

$$
m_{X \cdot v, \eta}\left(a \cdot z_{0}\right)=-a^{\alpha} \eta\left(Y a^{-1} \cdot v\right)=-a^{\alpha} m_{(\operatorname{Ad}(a) Y) \cdot v, \eta}\left(a \cdot z_{0}\right) .
$$

Inserting the root vector expansion (9.2), we obtain

$$
m_{X \cdot v, \eta}\left(a \cdot z_{0}\right)=-\sum_{\alpha+\beta \notin\langle I\rangle} a^{\alpha+\beta} m_{X_{\alpha, \beta} \cdot v, \eta}\left(a \cdot z_{0}\right) .
$$

By inserting for the matrix coefficients on the right-hand side of (9.3) their expansions according to $(6.17)$, we see that on $A_{I} \cap U_{A} A_{Z}^{-}$the function $m_{X \cdot v, \eta}\left(a \cdot z_{0}\right)$ allows an expansion with exponents which are of the form $\left.\xi\right|_{\mathfrak{a}_{I}}$ with $\xi=\xi^{\prime}+\sigma$, where $\xi^{\prime} \in \Xi$ and $\sigma=\alpha+\beta \in \mathbb{N}_{0}[S] \backslash\langle I\rangle$. By the definition of $\mathcal{E}_{\text {lead }, I}$ we get $\left.\xi\right|_{\mathfrak{a}_{I}} \neq \mu$, and hence we conclude that $\mathbf{L}_{I}^{\mu, \mathbf{1}}(a)(X v)=0$ for $a \in A_{I}$. Finally, the case of an arbitrary $w$ proceeds along the same lines in view of the description of $\mathfrak{h}_{w}$ in (3.20).

Let $W$ be a finite-dimensional vector space and $\mu \in\left(\mathfrak{a}_{I}\right)_{\mathbb{C}}^{*}$. Then we call $f: A_{I} \rightarrow W$ a $\mu$-polynomial map provided that for all $\lambda \in W^{*}$ there exists a polynomial $p_{\lambda}$ on $\mathfrak{a}_{I}$ such that $(\lambda \circ f)(a)=a^{\mu} p_{\lambda}(\log a)$ for all $a \in A_{I}$. Notice that there is a natural action of $A_{I}$ on the space of all $\mu$-polynomial maps, and accordingly we obtain an action of $\mathcal{U}\left(\mathfrak{a}_{I}\right)$. The following lemma is then straightforward from the definitions.

Lemma 9.3. Let $f: A_{I} \rightarrow W$ be a $\mu$-polynomial map.

(1) If $f \neq 0$, then there exists $u \in \mathcal{U}\left(\mathfrak{a}_{I}\right)$ such that $u \cdot f \neq 0$ and $(u \cdot f)(a)=a^{\mu}(u \cdot f)(\mathbf{1})$ for all $a \in A_{I}$.

(2) If in addition $W_{0} \subset W$ is a subspace with $\operatorname{im} f \subset W_{0}$, then $\operatorname{im}(u \cdot f) \subset W_{0}$ for all $u \in \mathcal{U}\left(\mathfrak{a}_{I}\right)$. 
We now apply this to the specific situation where $W=\left(V^{*}\right)^{\left(\mathfrak{h}_{w}\right)_{I}}$. Observe that $W \simeq$ $\left(V /\left(\mathfrak{h}_{w}\right)_{I} V\right)^{*}$ is a finite-dimensional $A_{I}$-module by Lemma 6.9, and that in Lemmas 9.1 and 9.2 we have established that $\mathbf{L}_{I}^{\mu, w}: A_{I} \rightarrow W$ is an $A_{I}$-equivariant $\mu$-polynomial map. There are two invariant subspaces which are in our interest: $\left(V^{-\infty}\right)^{\left(\mathfrak{h}_{w}\right)_{I}} \subset\left(V^{-\omega}\right)^{\left(\mathfrak{h}_{w}\right)_{I}}$. We already know from Lemma 9.3 that there exists an element $u \in \mathcal{U}\left(\mathfrak{a}_{I}\right)$ such that $u \cdot \mathbf{L}_{I}^{\mu, w}$ transforms as a character. Since we want to keep track of certain symmetries, we need to explicitly construct $u$ via an iterative procedure. This will be done in (9.12). To prepare for that, let $X \in \mathfrak{a}_{I}$ be such that $\sigma(X)<0$ for all $\sigma \in S \backslash I$ and sufficiently generic such that $\operatorname{Im} \lambda(X) \neq \operatorname{Im} \lambda^{\prime}(X)$ for all pairs $\lambda \neq \lambda^{\prime}$ in $\mathcal{E}_{\text {lead }, I}$ with $\operatorname{Re} \lambda(X)=\operatorname{Re} \lambda^{\prime}(X)$. Recall from $\S 6.3$ the constants $\lambda_{X}$ and $d_{X}$. Then $\operatorname{Re} \xi(X) \leqslant \lambda_{X}$ for all $\xi \in \Xi$, and for each $\xi \in \Xi$ with $\operatorname{Re} \xi(X)=\lambda_{X}$ we have $\left.\xi\right|_{\mathfrak{a}_{I}} \in \mathcal{E}_{\text {lead }, I}$. By Lemma 6.12 , the polynomial $t \mapsto q_{v, \xi, w}(Y+t X)$ has degree at most $d_{X}$ for all $Y \in \mathfrak{a}_{I}$. Hence, for each $\mu \in \mathcal{E}_{\text {lead }, I}$ with $\operatorname{Re} \mu(X)=\lambda_{X}$, an assignment

$$
\mathbf{L}_{I, X}^{\mu, w}: A_{I} \longrightarrow\left(V^{*}\right)^{\left(\mathfrak{h}_{w}\right)_{I}}
$$

is defined by

$$
\mathbf{L}_{I, X}^{\mu, w}(a)(v)=\lim _{t \rightarrow \infty} e^{-\mu(t X)} t^{-d_{X}} \mathbf{L}_{I}^{\mu, w}(a \exp (t X))(v) .
$$

Lemma 9.4. Let $X \in \mathfrak{a}_{I}$ be as above and $\mu \in \mathcal{E}_{\text {lead }, I}$ be such that $\operatorname{Re} \mu(X)=\lambda_{X}$. Suppose that $Z$ is either absolutely spherical or wave-front. Then, the following assertions hold for the $\mu$-polynomial map $\mathbf{L}_{I}^{\mu, w}: A_{I} \rightarrow\left(V^{*}\right)^{\left(\mathfrak{h}_{w}\right)_{I}}$ :

(1) $\operatorname{im} \mathbf{L}_{I, X}^{\mu, w} \subset\left(V^{-\infty}\right)^{\left(\mathfrak{h}_{w}\right)_{I}}$ for all $w$ and $\mu$ as above;

(2) $\mathbf{L}_{I, X}^{\mu, w}(\mathbf{1}) \neq 0$ for some $w$ and $\mu$.

Proof. (1) Suppose first that $Z$ is absolutely spherical. Fix $a \in A_{I}$ and $v \in V$. It follows from (6.30) that

$$
m_{v, \eta}\left(\exp (t X) a w \cdot z_{0}\right)=\sum_{\substack{\mu \in \mathcal{E}_{\text {lead }, I} \\ \operatorname{Re} \mu(X)=\lambda_{X}}} \mathbf{L}_{I}^{\mu, w}(a \exp (t X))(v)+o\left(e^{\lambda_{X} t}\right) .
$$

With $\eta_{\mu}:=\mathbf{L}_{I, X}^{\mu, w}(a)$, we then deduce from Theorem $7.2(1)$ and (9.4) that

$$
\left|\sum_{\substack{\mu \in \mathcal{E}_{\text {lead }, I} \\ \operatorname{Re} \mu(X)=\lambda_{X}}} e^{i \operatorname{Im} \mu(X) t} \eta_{\mu}(v)+R_{v}(t)\right| \leqslant q(v)
$$

for all $t \geqslant 1$, with remainder $R_{v}(t) \rightarrow 0$ for $t \rightarrow \infty$ and with a $G$-continuous norm $q$ on $V$. As we have chosen $X$ generic enough, we can use the oscillatory argument from [36, Lemma 4A.1.2, fact (1)] to deduce that

$$
\sum_{\mu}\left|\eta_{\mu}(v)\right|^{2} \leqslant \limsup _{t \rightarrow \infty}\left|\sum_{\mu} e^{i \operatorname{Im} \mu(X) t} \eta_{\mu}(v)\right|^{2},
$$


and hence $\left|\eta_{\mu}(v)\right| \leqslant q(v)$ for each $\mu$. This proves (1) for $Z$ absolutely spherical.

If $Z$ is wave-front, then we proceed similarly as above, but use Theorem 7.6 instead of Theorem 7.2(1) while carrying an additional $A_{Z}$-variable along: We first obtain as above for $b \in A_{I}$ that $\eta_{b}:=\mathbf{L}_{I, X}^{\mu, w}(b) \in\left(V^{-\omega}\right)^{\left(\mathfrak{h}_{w}\right)_{I}}$. Let $W_{\mu} \subset\left(V^{-\omega}\right)^{\left(\mathfrak{h}_{w}\right)_{I}}$ be the finite-dimensional space generated by $\operatorname{im} \mathbf{L}_{I, X}^{\mu, w}$. Observe that the action of $A_{I}$ on $W_{\mu}$ is by a single Jordan block corresponding to $\mu$. Hence, with the notions of Theorem 7.6 , we find a $d \in \mathbb{N}$ such that

$$
\left|m_{v, \eta_{b}}\left(\omega a w^{\prime} \cdot z_{w, I}\right)\right| \leqslant C_{\varepsilon} p_{\varepsilon}(v) b^{\mu} a^{\Lambda_{V, \eta}}(1+\|\log a\|+\|\log b\|)^{d}
$$

for all $v \in V, a \in A_{Z}^{-}, b \in A_{I}, \omega \in \Omega, w^{\prime} \in \mathcal{W}$ and all $\varepsilon>0$. It follows from [21, Lemma 3.1 and Proposition 3.4] that there exists a weight $\mathbf{w}: Z_{w, I} \rightarrow \mathbb{R}^{+}$such that

$$
\mathbf{w}\left(a b w^{\prime} \cdot z_{w, I}\right) \geqslant b^{\mu} a^{\Lambda_{V, \eta}}(1+\|\log a\|+\|\log b\|)^{d}
$$

for all $a \in A_{Z}^{-}, b \in A_{I}$ and $w^{\prime} \in \mathcal{W}$. Note that $A_{Z_{w, I}}^{-}=A_{I} A_{Z}^{-}$. In particular, employing the polar decomposition for $Z_{w, I}$, it follows that

$$
q(v):=\sup _{z \in Z_{w, I}}\left|m_{v, \eta_{b}}(z)\right| \mathbf{w}^{-1}(z)<\infty, \quad v \in V^{\omega} .
$$

We claim that $q$ is a $G$-continuous norm on $V$. To see that, we first note that the $G$-action on the Banach space $E=L^{\infty}\left(Z, \mathbf{w}^{-1} d z\right)$ is by locally equicontinuous operators (see [4, Lemma 2.3]), and hence the $G$-continuous vectors $E_{c} \subset E$ form a $G$-stable closed subspace of $E$, i.e. the action of $G$ on $E_{c}$ defines a Banach representation. Now $V \subset E_{c}$, as $V$ consists even of smooth vectors. Hence, $q$ is a $G$-continuous norm on $V$. Since $\left|\eta_{b}(v)\right| \leqslant \mathbf{w}\left(z_{w, I}\right) q(v)$ for all $v \in V$, we deduce that $\eta_{b} \in\left(V^{-\infty}\right)^{\left(\mathfrak{h}_{w}\right)_{I}}$.

To establish (2), we note that for each $\lambda \in \lambda_{X}+i \mathbb{R}$,

$$
e^{-\lambda t} \sum_{\substack{\mu \in \mathcal{E}_{\text {lead }, I} \\ \mu(X)=\lambda}} \mathbf{L}_{I}^{\mu, w}(\exp (t X))(v)=\sum_{\substack{\xi \in \Xi \\ \xi(X)=\lambda}} q_{v, \xi, w}(t X) .
$$

It follows from Lemma 6.12 that this polynomial in $t$ has degree $d_{X}$ for some $\lambda \in \lambda_{X}+i \mathbb{R}$ and some $v$ and $w$. Hence, $\mathbf{L}_{I}^{\mu, w}(\exp (t X))(v)$ has degree $d_{X}$ for some $\mu \in \mathcal{E}_{\text {lead }, I}$ with $\mu(X)=\lambda$. This implies that $\mathbf{L}_{I, X}^{\mu, w}(\mathbf{1}) \neq 0$.

We can equally implement $\mathbf{L}_{I, X}^{\mu, w}$ by a differential operator. To see that, we define for $Y \in \mathfrak{a}_{I}$ and a differentiable function $f$ on $A_{I}$,

$$
\partial_{Y} f(a):=(Y \cdot f)(a)=\left.\frac{d}{d t}\right|_{t=0} f(a \exp (t Y)) .
$$

Further, for $\gamma \in \mathfrak{a}_{I, \mathbb{C}}^{*}$, we define a first-order operator

$$
\partial_{\gamma, Y}:=e^{\gamma(\log (\cdot))} \circ \partial_{Y} \circ e^{-\gamma(\log (\cdot))}=\partial_{Y}-\gamma(Y) .
$$

The following lemma is then immediate. 
Lemma 9.5. Let $\mu$ and $w$ be as in (9.4). Then

$$
\left(\partial_{\mu, X}\right)^{d_{X}} \mathbf{L}_{I}^{\mu, w}=d_{X} ! \mathbf{L}_{I, X}^{\mu, w}
$$

For the rest of this article we assume that $Z$ is either absolutely spherical or wavefront.

Lemma 9.6. Let $w_{I} \in \mathcal{W}_{I}$, let $w \in \mathcal{W}$ correspond to $w_{I}$ as in Lemma 3.10, and let $\mu \in \mathcal{E}_{\text {lead }, I}$ with $\operatorname{Re} \mu(X)=\lambda_{X}$. There exists $m_{w} \in M$ such that

$$
m_{w} w_{I} \cdot \mathbf{L}_{I, X}^{\mu, \mathbf{1}}(a)=\mathbf{L}_{I, X}^{\mu, w}(a), \quad a \in A_{I}
$$

Proof. We give the proof for $Z$ absolutely spherical - the proof for $Z$ wave-front is analogous.

At first, let $w \in \mathcal{W}$ be arbitrary and $a_{s}=\exp (s X)$. Let $C_{I} \subset A_{I}$ be a compact subset. Then (9.5) implies that

$$
\sum_{\substack{\nu \in \mathcal{E}_{\text {lead }, I} \\ \operatorname{Re} \nu(X)=\operatorname{Re} \mu(X)}} e^{s(\nu-\mu)(X)} \mathbf{L}_{I, X}^{\nu, w}(a)=s^{-d_{X}} a_{s}^{-\mu}\left(a a_{s} \cdot \eta_{w}\right)+o\left(\frac{1}{s}\right) .
$$

The $o(1 / s)$ has to be understood in the following way: Let $q=q(X)$ be the $G$-continuous norm from Theorem $7.2(1)$ and $q^{*}$ be its dual norm. Then (9.6) means that

$$
\lim _{s \rightarrow \infty} q^{*}\left(s^{-d_{X}} a_{s}^{-\mu}\left(a a_{s} \cdot \eta_{w}\right)-\sum_{\substack{\nu \in \mathcal{E}_{\text {lead }, I} \\ \operatorname{Re} \nu(X)=\operatorname{Re} \mu(X)}} e^{s(\nu-\mu)(X)} \mathbf{L}_{I, X}^{\nu, w}(a)\right)=0
$$

uniformly for $a \in C_{I}$.

Moreover, note that Lemma 9.4 implies that

$$
\sup _{\substack{a \in C_{I} \\ s \in \mathbb{R}}} q^{*}\left(\sum_{\substack{\nu \in \mathcal{E}_{\operatorname{lead}, I} \\ \operatorname{Re} \nu(X)=\operatorname{Re} \mu(X)}} e^{s(\nu-\mu)(X)} \mathbf{L}_{I, X}^{\nu, w}(a)\right)<\infty .
$$

Now let $w$ correspond to $w_{I}$. From Lemma 3.10 we obtain that $w_{I} a_{s}=u_{s} m_{s} b_{s} w h_{s}$ with $u_{s} \in U, m_{s} \in M, b_{s} \in A_{Z}$ and $h_{s} \in H$ such that $u_{s} \rightarrow \mathbf{1}$ and $b_{s} a_{s}^{-1} \rightarrow \mathbf{1}$ for $s \rightarrow \infty$. Moreover, we may assume that $\left\{m_{s}\right\}_{s \geqslant s_{0}}$ is convergent, say with limit $m_{w}^{-1} \in M$. Then

$$
a_{s} w \cdot \eta=a_{s} b_{s}^{-1} m_{s}^{-1} u_{s}^{-1} w_{I} a_{s} \cdot \eta
$$

If we thus set $g_{s}:=a_{s} b_{s}^{-1} m_{s}^{-1} u_{s}^{-1}$, then $\left\{g_{s}\right\}_{s \geqslant s_{0}}$ is convergent to $m_{w}$ and

$$
a_{s} w \cdot \eta=g_{s} w_{I} a_{s} \cdot \eta
$$


From (9.7) we have

$$
\lim _{s \rightarrow \infty} q^{*}\left(s^{-d_{X}} a_{s}^{-\mu}\left(a a_{s} \cdot \eta\right)-\sum_{\substack{\nu \in \mathcal{E}_{\text {lead }, I} \\ \operatorname{Re} \nu(X)=\operatorname{Re} \mu(X)}} e^{s(\nu-\mu)(X)} \mathbf{L}_{I, X}^{\nu, \mathbf{1}}(a)\right)=0
$$

uniformly for $a \in C_{I}$. As $q^{*}$ is $G$-continuous and $\mathbf{L}_{I, X}^{\nu, \mathbf{1}}(a)$ is $H_{I}$-invariant, we hence obtain

$$
\lim _{s \rightarrow \infty} q^{*}\left(s^{-d_{X}} a_{s}^{-\mu}\left(w_{I} h a a_{s}\right) \cdot \eta-\sum_{\substack{\nu \in \mathcal{E}_{\text {lead }, I} \\ \operatorname{Re} \nu(X)=\operatorname{Re} \mu(X)}} e^{s(\nu-\mu)(X)}\left[\mathbf{L}_{I, X}^{\nu, \mathbf{1}}(a)\right]_{w_{I}}\right)=0
$$

uniformly for $h$ in compact subsets of $H_{I}$ and $a \in C_{I}$. Now note that there exists $h_{a} \in H_{I}$, depending continuously on $a \in A_{I}$, such that $w_{I} h_{a} a=a w_{I}$ (see Lemma 3.5). Hence, we get

$$
\lim _{s \rightarrow \infty} q^{*}\left(s^{-d_{X}} a_{s}^{-\mu}\left(a w_{I} a_{s}\right) \cdot \eta-\sum_{\substack{\nu \in \mathcal{E}_{\text {lead }, I} \\ \operatorname{Re} \nu(X)=\operatorname{Re} \mu(X)}} e^{s(\nu-\mu)(X))}\left[\mathbf{L}_{I, X}^{\nu, \mathbf{1}}(a)\right]_{w_{I}}\right)=0
$$

uniformly for $a \in C_{I}$. Further by applying $g_{s}$, which in the limit commutes with $a$, we deduce, with (9.8) and the $G$-continuity of $q^{*}$, that

$$
\lim _{s \rightarrow \infty} q^{*}\left(s^{-d_{X}} a_{s}^{-\mu}\left(a g_{s} w_{I} a_{s}\right) \cdot \eta-\sum_{\substack{\nu \in \mathcal{E}_{\text {lead }, I} \\ \operatorname{Re} \nu(X)=\operatorname{Re} \mu(X)}} e^{s(\nu-\mu)(X))} m_{w}\left[\mathbf{L}_{I, X}^{\nu, \mathbf{1}}(a)\right]_{w_{I}}\right)=0
$$

uniformly for $a \in C_{I}$. If we combine (9.10) with (9.9) and (9.7), we arrive at

$$
\lim _{s \rightarrow \infty} q^{*}\left(\sum_{\substack{\nu \in \mathcal{E}_{\operatorname{lead}, I} \\ \operatorname{Re} \nu(X)=\operatorname{Re} \mu(X)}} e^{s(\nu-\mu)(X)}\left(\mathbf{L}_{I, X}^{\nu, w}(a)-m_{w}\left[\mathbf{L}_{I, X}^{\nu, \mathbf{1}}(a)\right]_{w_{I}}\right)\right)
$$

uniformly for $a \in C_{I}$.

We are now in the position to apply Lemma 6.14. For that, note that it is no loss of generality to assume that the $G$-continuous norm $q$ from Theorem 7.2 is Hermitian (see [4]). Let $\mathcal{H}_{0}$ be the Hilbert completion of the Harish-Chandra module $\left(V^{-\infty}\right)^{K \text {-fin }}$ with respect to $q^{*}$. With $\mathcal{H}:=L^{2}\left(C_{I}, \mathcal{H}_{0}\right)$ the lemma follows now from (9.11) and Lemma 6.14, which is easily extended to a Hilbert-space-valued setting.

In the next step we produce out of $\mathbf{L}_{I, X}^{\mu, w}$ a continuous functional which transforms under the action of $A_{I}$ as a character. Note that

$$
\mathbf{L}_{I, X}^{\mu, w}(a)(v)=p_{v, \mu, w, X}(\log a) a^{\mu}
$$


where $p_{v, \mu, w, X}$ is a polynomial on $\mathfrak{a}_{I}$ which is constant along all affine rays $t \mapsto Y+t X$ in $\mathfrak{a}_{I}$. Now, if all $p_{v, \mu, w, X}$ are constant, then we set $\eta_{I}^{\mu, w}:=\eta_{I, X}^{\mu, w}$. Otherwise we set $X_{1}:=X$, choose $0 \neq X_{2} \in \mathfrak{a}_{I}$ orthogonal to $X_{1}$ and let $d_{2}$ be the maximum of the degrees of the polynomials $t \rightarrow p_{v, \mu, w, X_{1}}\left(Y+t X_{2}\right)$ for $Y \in \mathfrak{a}_{I}$ and $v \in V$. Then define, for $a \in A_{I}$ and $v \in V$,

$$
\mathbf{L}_{I, X_{1}, X_{2}}^{\mu, w}(a)(v)=\lim _{t \rightarrow \infty} t^{-d_{2}} e^{-t \mu\left(X_{2}\right)} \mathbf{L}_{I, X_{1}}^{\mu, w}\left(a \exp \left(t X_{2}\right)\right)(v) .
$$

As in Lemma 9.5, we have

$$
\mathbf{L}_{I, X_{1}, X_{2}}^{\mu, w}=\frac{1}{d_{2} !}\left(\partial_{\mu, X_{2}}\right)^{d_{2}} \mathbf{L}_{I, X_{1}}^{\mu, w} .
$$

With Lemmas 9.3 and 9.4, we thus get $\mathbf{L}_{I, X_{1}, X_{2}}^{\mu, w}(a) \in\left(V^{-\infty}\right)^{\left(\mathfrak{h}_{w}\right)_{I}}$. We iterate this process, and arrive at an element $\eta_{I}^{\mu, w} \in\left(V^{-\infty}\right)^{\left(\mathfrak{h}_{w}\right)_{I}}$ defined by

$$
\eta_{I}^{\mu, w}(v)=\mathbf{L}_{I, X_{1}, \ldots, X_{\mathrm{dim} \mathrm{a}_{I}}^{\mu, w}}(\mathbf{1})(v),
$$

with the additional property that it transforms under $A_{I}$ as a character:

$$
\eta_{I}^{\mu, w}(a v)=a^{\mu} \eta_{I}^{\mu, w}(v), \quad a \in A_{I} \text { and } v \in V
$$

Note that $\eta_{I}^{\mu, w} \neq 0$ if and only if $L_{I, X}^{\mu, w}(\mathbf{1}) \neq 0$. In particular, there exists $\mu \in \mathcal{E}_{\text {lead }, I}$ and $w \in \mathcal{W}$ such that the functional $\eta_{I}^{\mu, w}$ is non-zero. Further we note that

$$
m_{v, \eta_{I}^{\mu, w}}\left(a w \cdot z_{0, I}\right)=\sum_{\substack{\left.\xi \in \Xi \\ \xi\right|_{\mathfrak{I}_{I}}=\mu}} c_{v, \xi, w}(\log a) a^{\xi}, \quad a \in A_{Z}^{-} A_{I} \text { and } v \in V,
$$

with polynomials $c_{v, \xi, w}$ which do not depend on $\mathfrak{a}_{I}$.

Lemma 9.7. Let $w_{I} \in \mathcal{W}_{I}$ and let $w \in \mathcal{W}$ correspond to $w_{I}$ as in Lemma 3.10. There exists $m_{w} \in M$ such that

$$
m_{w} \cdot\left(\eta_{I}^{\mu, \mathbf{1}}\right)_{w_{I}}=\eta_{I}^{\mu, w} .
$$

Proof. In view of the construction of $\eta_{I}^{\mu, w}$, this is now immediate from Lemmas 9.6 and 9.5.

\subsection{The tempered embedding theorem}

Let $(V, \eta)$ be an $H$-spherical tempered pair. Then $\varrho_{Q}\left(\omega_{i}\right) \leqslant \Lambda_{V, \eta}\left(\omega_{i}\right)$ for $i=1, \ldots, s$, by Theorem 8.2. For each leading exponent $\lambda$ we have $\Lambda_{V, \eta}\left(\omega_{i}\right) \leqslant \operatorname{Re} \lambda\left(\omega_{i}\right)$ by definition, and hence

$$
\varrho_{Q}\left(\omega_{i}\right) \leqslant \Lambda_{V, \eta}\left(\omega_{i}\right) \leqslant \operatorname{Re} \lambda\left(\omega_{i}\right)
$$

We attach to $\lambda$ the following set of spherical roots:

$$
I_{\eta, \lambda}:=\left\{\sigma_{i} \in S: \varrho_{Q}\left(\omega_{i}\right)<\operatorname{Re} \lambda\left(\omega_{i}\right)\right\} .
$$


LEMMA 9.8. For $\lambda \in \mathcal{E}_{\text {lead }}$ and $I=I_{\eta, \lambda}$ the following assertions hold:

(1) $\left.\varrho_{Q}\right|_{\mathfrak{a}_{I}}=\left.\Lambda_{V, \eta}\right|_{\mathfrak{a}_{I}}=\left.\operatorname{Re} \lambda\right|_{\mathfrak{a}_{I}}$;

(2) for all $\lambda^{\prime} \in \mathcal{E}_{\text {lead }}$ and $\sigma_{i} \notin I$ one has $\operatorname{Re}\left(\lambda^{\prime}-\lambda\right)\left(\omega_{i}\right) \geqslant 0$;

(3) $\left.\lambda\right|_{\mathfrak{a}_{I}} \in \mathcal{E}_{\text {lead }, I}$;

(4) if $\lambda^{\prime} \in \mathcal{E}_{\text {lead }}$ and $\left.\operatorname{Re} \lambda^{\prime}\right|_{\mathfrak{a}_{I}}=\left.\operatorname{Re} \lambda\right|_{\mathfrak{a}_{I}}$, then $I_{\eta, \lambda^{\prime}} \subset I_{\eta, \lambda}$.

Proof. (1) follows from (9.16) and (9.17), since $\mathfrak{a}_{I}$ is spanned by the $\omega_{i}$ for $\sigma_{i} \notin I$. Next, (2) follows from (1) together with (9.16). Moving on to (3), we argue by contradiction and assume that $\left.\lambda\right|_{\mathfrak{a}_{I}} \notin \mathcal{E}_{\text {lead,I}}$. Then there exist an $\gamma \in \mathcal{E}_{\text {lead }, I}$ and a $\sigma \in \mathbb{N}_{0}[S] \backslash \mathbb{N}_{0}[I]$ such that $\left.(\lambda-\sigma)\right|_{\mathfrak{a}_{I}}=\gamma$. Since $\left.\sigma\right|_{\mathfrak{a}_{I}} \neq 0$, this contradicts (2). Finally, (4) is obvious from the definition.

Let $\min _{\eta}:=\min \left\{\# I_{\eta, \lambda}: \lambda \in \mathcal{E}_{\text {lead }}\right\}$.

Lemma 9.9. Let $(V, \eta)$ be an $H$-spherical tempered pair. Then there exists a $\lambda \in \mathcal{E}_{\text {lead }}$ such that, for $I=I_{\eta, \lambda}$ and $\mu=\left.\lambda\right|_{\mathfrak{a}_{I}}$, the following assertions hold:

(1) $\# I=\min _{\eta}$;

(2) $\eta_{I}^{\mu, w} \neq 0$ for some $w \in \mathcal{W}$.

Proof. Let $\gamma \in \mathcal{E}_{\text {lead }}$ be such that assertion (1) holds for $I:=I_{\eta, \gamma}$. According to Lemma $9.8(3)$, we have $\left.\gamma\right|_{\mathfrak{a}_{I}} \in \mathcal{E}_{\text {lead }, I}$. For all $X \in \mathbf{S}\left(\mathfrak{a}_{Z}^{-}\right)$we have $\operatorname{Re} \gamma(X) \leqslant \lambda_{X} \leqslant \Lambda_{V, \eta}(X)$. If in addition $X \in \mathfrak{a}_{I}$, then Lemma 9.8 (1) implies $\operatorname{Re} \gamma(X)=\lambda_{X}=\Lambda_{V, \eta}(X)$.

Let $X \in \mathfrak{a}_{I}$ be the sufficiently generic element used in the construction of $\mathbf{L}_{I}^{\mu, w}$ (above Lemma 9.4). By this construction and the one above Lemma 9.7, we infer that there exists a $\lambda \in \mathcal{E}_{\text {lead }}$ with $\operatorname{Re} \lambda(X)=\lambda_{X}$ such that for some $w \in \mathcal{W}$ we have $\eta_{I}^{\mu, w} \neq 0$, where $\mu:=\left.\lambda\right|_{\mathfrak{a}_{I}}$. It follows from (1) and Lemma $9.8(4)$ that $I_{\lambda, \eta}=I$. Hence the assertion follows.

Pairs $(\lambda, w) \in \mathcal{E}_{\text {lead }} \times \mathcal{W}$ which satisfy the conditions of Lemma 9.9 will be called $\eta$-optimal.

Recall that $\mathfrak{a}_{Z_{I}}=\mathfrak{a}_{Z}$ and $\mathfrak{a}_{Z_{I}, E}=\mathfrak{a}_{I}$. For $\sigma \in I$ we define $\omega_{\sigma, I} \in \mathfrak{a}_{Z}$ by

- $\sigma^{\prime}\left(\omega_{\sigma, I}\right)=\delta_{\sigma \sigma^{\prime}}$ for $\sigma^{\prime} \in I$;

- $\omega_{\sigma, I} \perp \mathfrak{a}_{I}$.

We note that $\omega_{\sigma, S}$ coincides with the previously defined $\omega_{\sigma}$. In general,

$$
\omega_{\sigma, I}-\omega_{\sigma} \in \mathfrak{a}_{I}, \quad \sigma \in I
$$

Let now $(\lambda, w) \in \mathcal{E}_{\text {lead }} \times \mathcal{W}$ be $\eta$-optimal, $I=I_{\eta, \lambda}$ and $\mu=\left.\lambda\right|_{\mathfrak{a}_{I}}$. We then define a linear functional $\Lambda_{V, \eta, I} \in \mathfrak{a}_{Z}^{*}$ by the following requirements:

- $\left.\left(\Lambda_{V, \eta, I}-\varrho_{Q}\right)\right|_{\mathfrak{a}_{I}}=0$;

- $\Lambda_{V, \eta, I}\left(\omega_{\sigma, I}\right)=\min \left\{\operatorname{Re} \gamma\left(\omega_{\sigma, I}\right): \gamma \in \mathcal{E}_{\text {lead }}\right.$ and $\left.\left.\operatorname{Re} \gamma\right|_{\mathfrak{a}_{I}}=\mu\right\}$ for $\sigma \in I$. 
Lemma 9.10. Let $(V, \eta)$ be a $Z$-tempered and $(\lambda, w) \in \mathcal{E}_{\text {lead }} \times \mathcal{W}$ be an associated $\eta$-optimal pair. Then

$$
\left(\Lambda_{V, \eta, I}-\varrho_{Q}\right)\left(\omega_{\sigma, I}\right)>0, \quad \sigma \in I .
$$

Proof. In view of the definition of $\Lambda_{V, \eta, I}$ and (9.18), it is sufficient to check that $\left(\Lambda_{V, \eta, I}-\varrho_{Q}\right)\left(\omega_{\sigma}\right)>0$ for all $\sigma \in I$. Let now $\gamma \in \mathcal{E}_{\text {lead }}$ be such that $\left.\operatorname{Re} \gamma\right|_{\mathfrak{a}_{I}}=\mu$. Then $I_{\eta, \gamma}=I$ by Lemma $9.8(4)$ and the minimality of \#I. Hence, $\left(\operatorname{Re} \gamma-\varrho_{Q}\right)\left(\omega_{\sigma}\right)>0$ by the definition of $I_{\eta, \gamma}$.

TheOREM 9.11. Suppose that $Z$ is either absolutely spherical or of wave-front type. Let $(V, \eta)$ be an $H$-spherical tempered pair with $V$ irreducible. Then, for every $\eta$ optimal pair $(\lambda, w) \in \mathcal{E}_{\text {lead }} \times \mathcal{W}$, there exist a boundary degeneration $Z_{w, I}=G /\left(H_{w}\right)_{I}$ of $Z_{w}=G / H_{w}$, a normalized unitary character $\xi$ of $\left(H_{w}\right)_{I} A_{I}$ and $a(\mathfrak{g}, K)$-embedding

$$
V \hookrightarrow L^{2}\left(\widehat{Z}_{w, I} ; \xi\right)
$$

where $\widehat{Z}_{w, I}:=G /\left(H_{w}\right)_{I} A_{I}$. In particular, $V$ is unitarizable.

Proof. Let $(\lambda, w) \in \mathcal{E}_{\text {lead }} \times \mathcal{W}$ be $\eta$-optimal, and let $I=I_{\eta, \lambda}$ and $\mu=\left.\lambda\right|_{\mathfrak{a}_{I}}$. Then $\eta_{I}:=$ $\eta_{I}^{\mu, w} \neq 0$, and recall that it is a continuous $\left(H_{w}\right)_{I}$-invariant functional. Hence, after replacing $H$ by $H_{w}$, we may assume that $w=\mathbf{1}$.

As $V$ is irreducible, the map

$$
\begin{gathered}
\Phi: V^{\infty} \longrightarrow C^{\infty}\left(Z_{I}\right), \\
v \longmapsto m_{v, \eta_{I}},
\end{gathered}
$$

defines a $G$-equivariant injection. Notice that, by (9.13), all elements in im $\Phi$ transform under the normalized unitary character $a^{\mu}$ by the right $A_{I}$-action.

Recall the set $\mathcal{W}_{I}=\left\{w_{1, I}, \ldots, w_{p, I}\right\}$. For $1 \leqslant i \leqslant p$ let $w_{i} \in \mathcal{W}$ correspond to $w_{i, I}$ as in Lemma 3.10. From (9.15) we get $m_{j} \cdot\left(\eta_{I}\right)_{w_{j, I}}=\eta_{I}^{\mu, w_{j}}$ for $j=1, \ldots, p$ and some $m_{j} \in M$. Hence,

$$
m_{\eta_{I}, v}\left(a w_{j, I} \cdot z_{0, I}\right)=m_{\eta_{I}^{\mu, w_{j}, v}}\left(m_{j} a \cdot z_{0, w_{j}, I}\right)
$$

for all $a \in A_{Z}$. Here $z_{0, w_{j}, I}$ denotes the origin of $G /\left(H_{w_{j}}\right)_{I}$. It follows from (9.14), applied to the matrix coefficient on the right-hand side of (9.20), that all exponents belong to $\Xi$ and restrict to $\mu$ on $\mathfrak{a}_{I}$. This being the case for all $j=1, \ldots, p$, we then infer from the definition of $\Lambda_{V, \eta, I}$ that

$$
\Lambda_{V, \eta_{I}}\left(\omega_{\sigma, I}\right) \geqslant \Lambda_{V, \eta, I}\left(\omega_{\sigma, I}\right), \quad \sigma \in I,
$$

for the exponent defined by (6.20) for $\eta_{I}$.

The theorem now follows by applying Theorem 8.5 to $Z_{I}$ (see also Lemma 3.12), together with inequality (9.19). 
Remark 9.12. After a change of $P_{\min }($ and $A$ ) it is possible to get an optimal pair $(\lambda, w)$ such that $w=\mathbf{1}$. In order to see that, note that

$$
m_{v, \eta_{w}}\left(a \cdot z_{w}\right)=m_{w^{-1} v, \eta}\left(w^{-1} a w \cdot z_{0}\right) .
$$

We are allowed to change $\left(A, K, P_{\min }\right)$ to $\operatorname{Ad}\left(w^{-1}\right)\left(A, K, P_{\min }\right)$ without effect on the developed theory of power series expansions. The asserted reduction to $w=\mathbf{1}$ follows.

Corollary 9.13. Assume that $Z$ is wave-front and let $(V, \eta),(\lambda, w)$ be as above. Then there exist

(1) a parabolic subgroup $P \supset \bar{Q}$,

(2) a Levi decomposition $P=G_{P} U_{P}$,

(3) a real spherical subgroup $H_{P}^{\prime} \subset G_{P}$ containing $\left(G_{P} \cap H_{w}\right)_{e}$,

(4) a twisted discrete series representation $\sigma$ of $G_{P} / H_{P}^{\prime}$

such that $V$ embeds equivariantly into the parabolically induced unitary representation $\operatorname{Ind}_{P}^{G}(\sigma \otimes 1)$.

Proof. Let $I$ be as in Theorem 9.11, and let $P=\bar{P}_{F}$ be as in Corollary 5.6, that is, $\bar{U}_{F} \subset H_{I} \subset \bar{P}_{F}$. In view of Lemma 3.7, we have $\bar{U}_{F} \subset\left(H_{w}\right)_{I} \subset \bar{P}_{F}$ as well. Hence, the assertion follows with $H_{P}^{\prime}=\left(G_{F} \cap\left(H_{w}\right)_{I}\right)_{e}$ from Lemma 8.7.

Example 9.14. Let $G / H$ be a symmetric space as in Example 3.4. Then $Z$ is wavefront.

After possible change of $P$ to $w^{-1} P w$ (see Remark 9.12), we may assume that there is a $\lambda \in \mathcal{E}_{\text {lead }}$ such that $(\lambda, \mathbf{1})$ is an $\eta$-optimal pair. After a further change of $P$ to $h P h^{-1}$ for some $h \in H$ (see [30, Theorem 1]), we may assume that $P$ is a $\sigma \theta$-stable parabolic subgroup. Then $H_{P}^{\prime}=\left(G_{P}^{\sigma}\right)_{e}$ is a symmetric subgroup in $G_{P}$. Hence, Corollary 9.13 implies Delorme's result from [12] (up to components of $G_{P}^{\sigma}$ ). The result of Langlands cited in the introduction then also follows.

\section{References}

[1] Aizenbud, A., Gourevitch, D., Krötz, B. \& Liu, G., Hausdorffness for Lie algebra homology of Schwartz spaces and applications to the comparison conjecture. Math. Z., 283 (2016), 979-992, 993-994.

[2] VAN DEN BAN, E.P., Asymptotic behaviour of matrix coefficients related to reductive symmetric spaces. Nederl. Akad. Wetensch. Indag. Math., 49 (1987), 225-249.

[3] VAN DEN BAN, E. P. \& Delorme, P., Quelques propriétés des représentations sphériques pour les espaces symétriques réductifs. J. Funct. Anal., 80 (1988), 284-307.

[4] Bernstein, J. \& Krötz, B., Smooth Fréchet globalizations of Harish-Chandra modules. Israel J. Math., 199 (2014), 45-111.

[5] Bernstein, J. \& Reznikov, A., Estimates of automorphic functions. Mosc. Math. J., 4 (2004), 19-37. 
[6] Bratten, T., A comparison theorem for Lie algebra homology groups. Pacific J. Math., 182 (1998), 23-36.

[7] Brion, M., Classification des espaces homogènes sphériques. Compositio Math., 63 (1987), 189-208.

[8] Brylinski, J.-L. \& Delorme, P., Vecteurs distributions $H$-invariants pour les séries principales généralisées d'espaces symétriques réductifs et prolongement méromorphe d'intégrales d'Eisenstein. Invent. Math., 109 (1992), 619-664.

[9] Bunke, U. \& Olbrich, M., Cohomological properties of the canonical globalizations of Harish-Chandra modules. Ann. Global Anal. Geom., 15 (1997), 401-418.

[10] Casselman, W. \& Miličić, D., Asymptotic behavior of matrix coefficients of admissible representations. Duke Math. J., 49 (1982), 869-930.

[11] Danielsen, T., Krötz, B. \& Schlichtkrull, H., Decomposition theorems for triple spaces. Geom. Dedicata, 174 (2015), 145-154.

[12] Delorme, P., Injection de modules sphériques pour les espaces symétriques réductifs dans certaines représentations induites, in Noncommutative Harmonic Analysis and Lie Groups (Marseille-Luminy, 1985), Lecture Notes in Math., 1243, pp. 108-143. Springer, Berlin-Heidelberg, 1987.

[13] Eskin, A. \& McMullen, C., Mixing, counting, and equidistribution in Lie groups. Duke Math. J., 71 (1993), 181-209.

[14] Flensted-Jensen, M., Spherical functions of a real semisimple Lie group. A method of reduction to the complex case. J. Funct. Anal., 30 (1978), 106-146.

[15] Gross, B. H. \& Prasad, D., On the decomposition of a representation of $\mathrm{SO}_{n}$ when restricted to $\mathrm{SO}_{n-1}$. Canad. J. Math., 44 (1992), 974-1002.

[16] Knapp, A. W., Representation Theory of Semisimple Groups. Princeton Landmarks in Mathematics. Princeton University Press, Princeton, NJ, 2001.

[17] Knop, F. \& Krötz, B., Reductive group actions. Preprint, 2016. arXiv: 1604.01005v2 [math.RT].

[18] Knop, F., Krötz, B., Pecher, T. \& Schlichtkrull, H., Classification of reductive real spherical pairs I: The simple case. To appear in Transform. Groups.

[19] - Classification of reductive real spherical pairs II: The semisimple case. Preprint, 2017. arXiv: 1703.08048 [math.RT].

[20] Knop, F., Krötz, B., Sayag, E. \& Schlichtkrull, H., Simple compactifications and polar decomposition of homogeneous real spherical spaces. Selecta Math., 21 (2015), 1071-1097.

[21] — Volume growth, temperedness and integrability of matrix coefficients on a real spherical space. J. Funct. Anal., 271 (2016), 12-36.

[22] Knop, F., Krötz, B. \& Schlichtkruld, H., The local structure theorem for real spherical varieties. Compos. Math., 151 (2015), 2145-2159.

[23] KRÄMER, M., Sphärische Untergruppen in kompakten zusammenhängenden Liegruppen. Compositio Math., 38 (1979), 129-153.

[24] Krötz, B., Sayag, E. \& Schlichtkrull, H., Decay of matrix coefficients on reductive homogeneous spaces of spherical type. Math. Z., 278 (2014), 229-249.

[25] - The harmonic analysis of lattice counting on real spherical spaces. Doc. Math., 21 (2016), 627-660.

[26] Krötz, B. \& Schlichtkrull, H., Finite orbit decomposition of real flag manifolds. J. Eur. Math. Soc. (JEMS), 18 (2016), 1391-1403.

[27] - Multiplicity bounds and the subrepresentation theorem for real spherical spaces. Trans. Amer. Math. Soc., 368 (2016), 2749-2762. 
[28] Langlands, R. P., On the classification of irreducible representations of real algebraic groups, in Representation Theory and Harmonic Analysis on Semisimple Lie Groups, Math. Surveys Monogr., 31, pp. 101-170. Amer. Math. Soc., Providence, RI, 1989.

[29] Mackey, G. W., Induced representations of locally compact groups. I. Ann. of Math., 55 (1952), 101-139.

[30] Matsuki, T., The orbits of affine symmetric spaces under the action of minimal parabolic subgroups. J. Math. Soc. Japan, 31 (1979), 331-357.

[31] - Orbits on flag manifolds, in Proceedings of the International Congress of Mathematicians (Kyoto, 1990), Vol. II, pp. 807-813. Math. Soc. Japan, Tokyo, 1991.

[32] Mikityuk, I. V., Integrability of invariant Hamiltonian systems with homogeneous configuration spaces. Mat. Sb., 129 (1986), 514-534 (Russian); English translation in Math. USSR Sb., 57 (1987), 527-546.

[33] Rossmann, W., The structure of semisimple symmetric spaces. Canad. J. Math., 31 (1979), 157-180.

[34] Sakellaridis, Y. \& Venkatesh, A., Periods and harmonic analysis on spherical varieties. To appear in Astérisque.

[35] van Steirteghem, B., Various interpretations of the root system(s) of a spherical variety. Oberwolfach Reports, 10 (2013), 1464-1467.

[36] Wallach, N. R., Real Reductive Groups. I. Pure and Applied Mathematics, 132. Academic Press, Boston, MA, 1988.

FRIEDRICH KNOP

Department Mathematik

Emmy-Noether Zentrum

FAU Erlangen-Nürnberg, Cauerstr. 11

DE-91058 Erlangen

Germany

friedrich.knop@fau.de

HENRIK SchlichtKRULL

University of Copenhagen

Department of Mathematics

Universitetsparken 5

DK-2100 Copenhagen $\varnothing$

Denmark

schlicht@math.ku.dk

Received September 30, 2015

Received in revised form August 12, 2016
BERnhaRd KRÖTZ

Universität Paderborn

Institut für Mathematik

Warburger Straße 100

DE-33098 Paderborn

Germany

bkroetz@gmx.de 